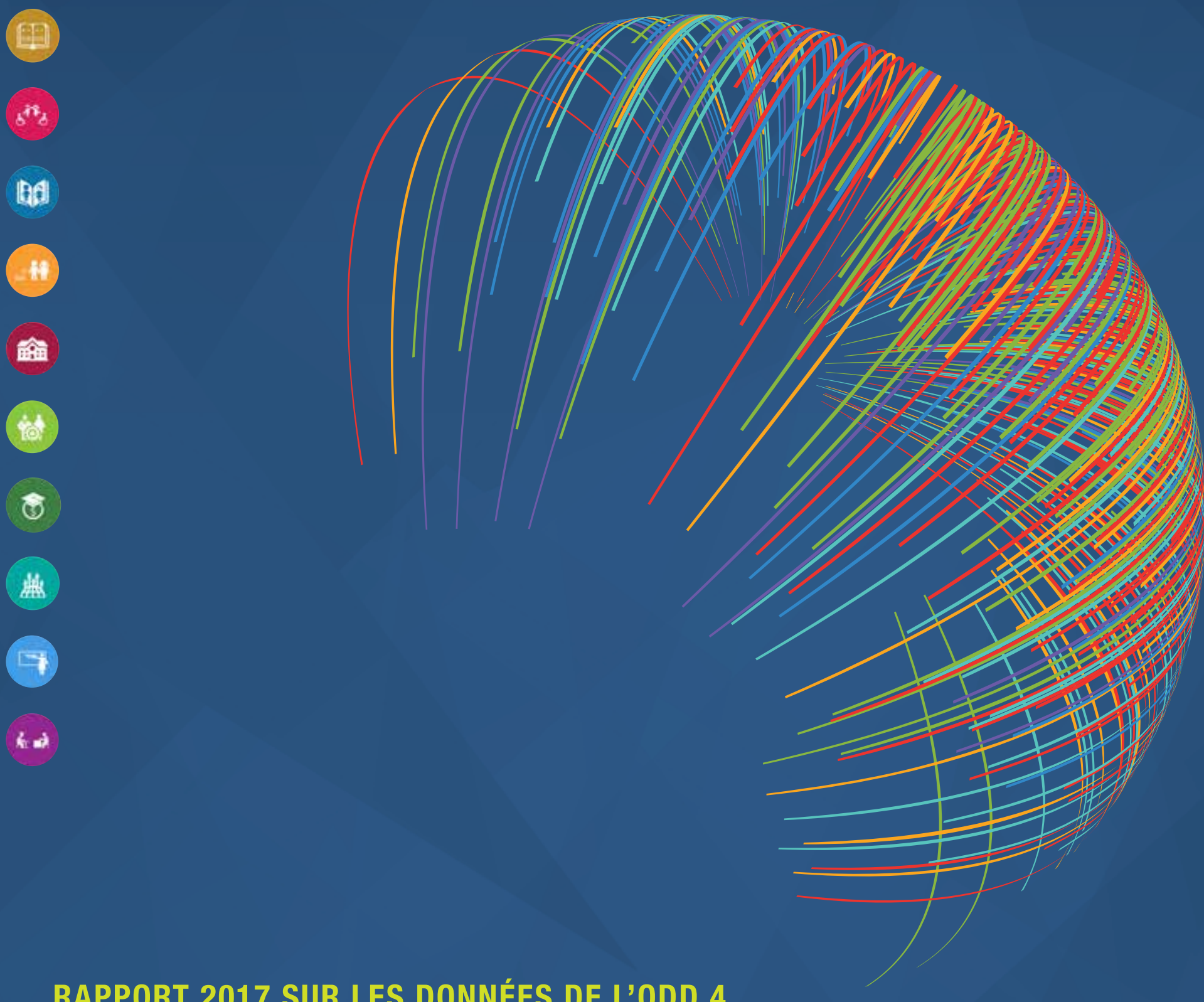

RAPPORT 2017 SUR LES DONNÉES DE L'ODD 4

\title{
Le facteur qualité : Renforcer les données nationales afin de surveiller l'Objectif de développement durable 4
}

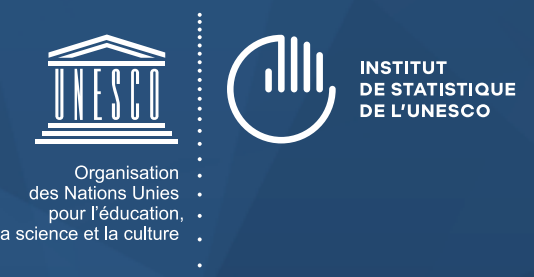
OBJECTIFS
DEDEEEOPPEMENT
DURABLE 

RAPPORT 2017 SUR LES DONNÉES DE L'ODD 4

Le facteur qualité : Renforcer les

données nationales afin de surveiller

l'Objectif de développement durable 4

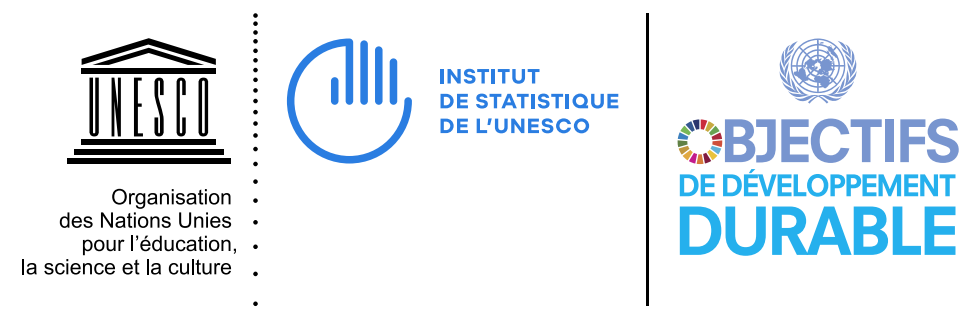




\section{UNESCO}

L'Acte constitutif de l'Organisation des Nations Unies pour l'éducation, la science et la culture (UNESCO) a été adopté par 20 pays lors de la Conférence de Londres en novembre 1945 et est entré en vigueur le 4 novembre 1946. L'UNESCO compte actuellement 195 États membres et 10 membres associés.

L'objectif premier de l'UNESCO est de contribuer au maintien de la paix et de la sécurité dans le monde en resserrant, par l'éducation, la science et la culture, la collaboration entre nations afin d'assurer le respect universel de la justice, de la loi, des droits de l'homme et des libertés fondamentales pour tous, sans distinction de race, de sexe, de langue ou de religion, que la Charte des Nations Unies reconnaît à tous les peuples.

L'UNESCO a cinq fonctions principales, inscrites dans son mandat : 1) des études prospectives sur l'éducation, la science, la culture et la communication dans le monde de demain ; 2 ) le progrès, le transfert et le partage des connaissances par des activités de recherche, de formation et d'enseignement ; 3) des actions normatives en vue de la préparation et de l'adoption d'instruments internationaux et de recommandations réglementaires ; 4) l'expertise par le biais de la coopération technique avec les États membres, en faveur de leurs projets et politiques de développement ;

5) l'échange d'informations spécialisées.

\section{Institut de statistique de l'UNESCO}

L'Institut de statistique de l'UNESCO (ISU) est la source officielle des données comparables au plan international utilisées pour surveiller les progrès réalisés sur la voie de l'Objectif de développement durable lié à l'éducation (ODD 4) et des principales cibles en rapport avec la science et la culture. Comme l'a confirmé le Cadre d'action Éducation 2030-ODD 4, l'ISU a pour mandat de « travailler avec ses partenaires pour élaborer de nouveaux indicateurs, approches statistiques et outils de suivi afin de mieux évaluer les progrès réalisés sur la voie des cibles liées au mandat de I'UNESCO ».

L'ISU a été créé en 1999. II a été conçu pour améliorer le programme statistique de l'UNESCO et pour élaborer et fournir en temps voulu les statistiques exactes et pertinentes d'un point de vue politique dont nous avons besoin dans un monde de plus en plus complexe et au sein duquel ne cessent de changer les environnements sociaux, politiques et économiques.

Publié en 2018 par :

Institut de statistique de I'UNESCO

C.P. 6128 , Succursale Centre-Ville

Montréal, Québec H3C 3J7

Canada

Téléphone : (1 514) 343-6880

Courriel : uis.publications@unesco.org

http://www.uis.unesco.org

ISBN : 978-92-9189-226-6

Réf : UIS/2017/ED/SD/15

CUNESCO-UIS 2018

Cette publication est disponible en accès libre sous la licence Attribution-ShareAlike 3.0 IGO (CC-BY-SA 3.0 IGO) (http://creativecommons.org/licenses/by-sa/3.0/igo/). En utilisant le contenu de cette publication, les utilisateurs acceptent de se conformer aux conditions d'utilisation du service d'archive des publications en accès libre de l'UNESCO (http://fr.unesco.org/open-access/terms-use-ccbysa-fr).

Les appellations employées dans cette publication et la présentation des données qui y figurent n'impliquent de la part de l'UNESC0 aucune prise de position quant au statut juridique de tout pays, territoire, ville ou zone ou de leurs autorités, ni quant au tracé de leurs frontières ou limites.

Les idées et opinions exprimées dans cette publication sont celles des auteurs. Elles ne représentent pas nécessairement les vues de l'UNESCO et n'engagent à rien l'Organisation. 


\section{Table des matières}

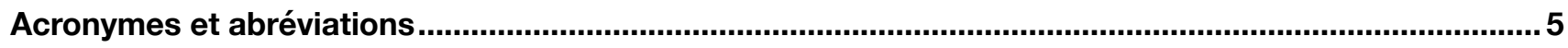

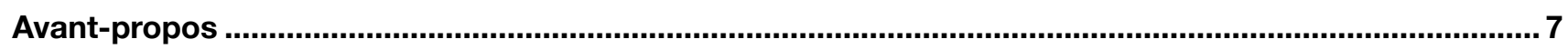

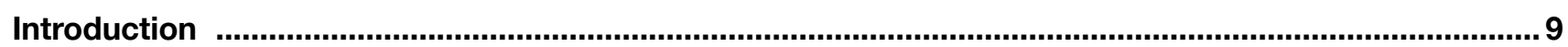

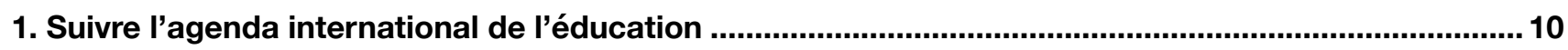

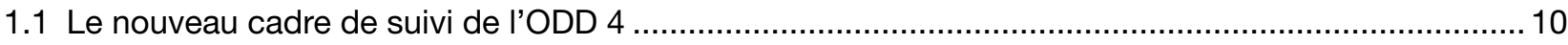

1.2 L'élaboration et la mise en œuvre des cadres d'indicateurs mondiaux et thématiques de l'ODD $4 \ldots 12$

1.2.1 Le cadre des indicateurs mondiaux................................................................................... 13

1.2.2 Le cadre des indicateurs thématiques ........................................................................... 18

1.3 Le défi de la production des données requises pour les indicateurs ............................................. 19

2. Produire des statistiques de qualité pour surveiller les progrès réalisés sur la voie de l'ODD $4 \ldots . . . . .23$

2.1 Une révolution des données sur l'éducation ............................................................................. 23

2.2 Produire des données sur l'éducation au niveau national conformément aux normes de qualité.........27

2.2.1 Définir et mettre en œuvre les normes.................................................................................... 27

2.2.2 Utiliser les cadres d'évaluation de la qualité des données pour les statistiques

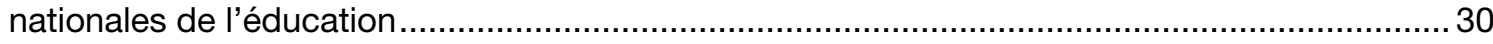

2.3 Produire des indicateurs de l'ODD 4 de qualité pour le suivi mondial .............................................. 34

2.3.1 Élaboration de définitions et de normes internationales en vue de la production

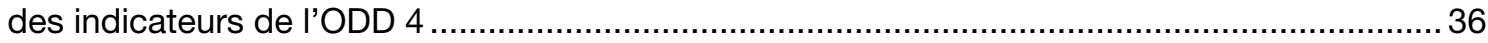

2.3.2 Collecte des données, traitement et validation................................................................... 38

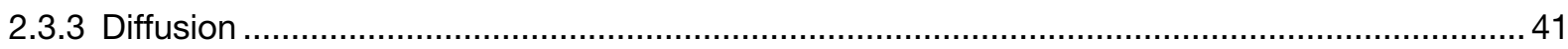

3. Soutenir le développement des capacités statistiques nationales afin de surveiller l'ODD $4 \ldots . . . . . . . . . .42$

3.1 Concevoir et mettre en œuvre une Stratégie nationale de l'élaboration des statistiques

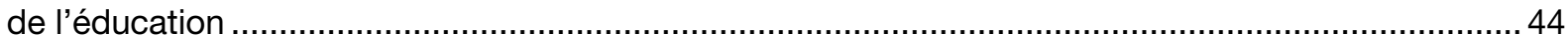

3.2 Recenser les sources de données et la disponibilité des informations .............................................46

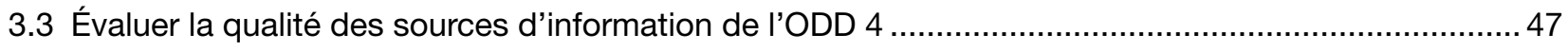

3.3.1 Analyse de la qualité des données de systèmes administratifs courants .............................. 48

3.3.2 Analyse de la qualité des données pour les sources des données relatives aux

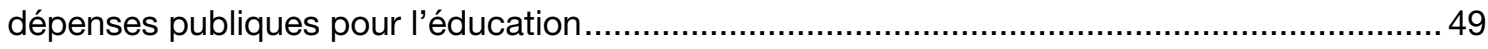

3.3.3 Analyse de la qualité des données des enquêtes auprès des ménages .................................50

3.3.4 Analyse de la qualité des données des évaluations des résultats d'apprentissage ..................51

3.4 Vers une approche coordonnée de la production des données sur l'éducation.................................52

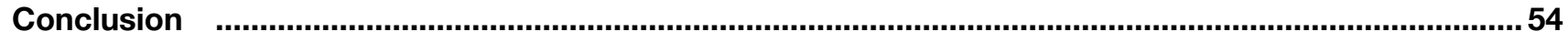

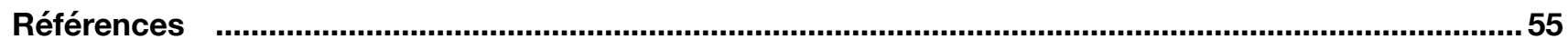

Annexe 1. Code de pratiques pour les statistiques de l'éducation : Principes et indicateurs ........................59

Annexe 2. Liens entre le Code de pratiques de I'ISU pour les statistiques de l'éducation et

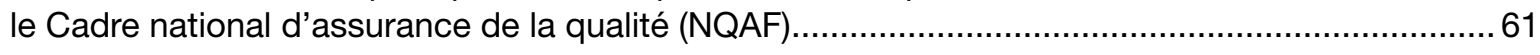

Annexe 3. Stratégies de mise en œuvre de développement des capacités nationales................................. 63 
Annexe 4. Cibles de l'ODD 4 par source des données

Annexe 5. Douze questions principales pour évaluer la qualité des données des systèmes de finances publiques

\section{Liste des tableaux}

Tableau 1. Statut actuel des indicateurs mondiaux et thématiques du suivi de l'ODD 4

Tableau 2. Extrait du Modèle de rapport sur les résultats du questionnaire relatif au recensement des données de l'ODD 4.

Tableau 3. Indicateurs de l'ODD 4 par source de données.

\section{Liste des figures}

Figure 1. Les quatre niveaux du suivi des cibles liées à l'éducation.

Figure 2. Disponibilité des indicateurs mondiaux et thématiques de l'ODD 4 dans la base de données de l'ISU, juin 2017 .

Figure 3. Composantes de la révolution des données sur l'éducation

Figure 4. Structure de l'Ed-DQAF à des fins administratives.

Figure 5. Stratégie de l'ISU à moyen terme, pour 2017-2021 35

Figure 6. Flux de données pour produire les indicateurs mondiaux de l'ODD 4 Data 36

Figure 7. Assurance de la qualité lors de la collecte, du traitement et de la validation effectués par I'ISU......39

Figure 8. Modèle de développement des capacités 42

Figure 9. Organisation des Stratégies nationales de développement des statistiques (SNDS).....

Figure 10. Composantes de I'ISU pour soutenir la production et les outils nationaux des données sur l'éducation

\section{Liste des encadrés}

Encadré 1. Catégorisation des indicateurs mondiaux de I'ODD 4 grâce aux niveaux du GIAE-ODD. 17

Encadré 2. Critères de validation des données de qualité.........................................................................22

Encadré 3. Un code de bonnes pratiques pour les statistiques, en Amérique latine et aux Caraïbes ..............26

Encadré 4. Le Cadre national d'assurance de la qualité des Nations Unies (NQAF) et le Cadre d'assurance de la qualité des statistiques des Nations Unies (UN SQAF) ..................26

Encadré 5. Cadres nationaux d'assurance de la qualité des données : Le cas de la Colombie ......................27

Encadré 6. Les 12 principes du Code de pratiques des statistiques de l'éducation......................................29

Encadré 7. Listes de contrôle utilisées pendant les évaluations de la qualité : L'exemple du Principe 8 .........33

Encadré 8. La Classification internationale type de l'éducation (CITÉ) .......................................................37

Encadré 9. Défis de la production des indicateurs des résultats d'apprentissage ........................................40

Encadré 10. Le suivi des plans nationaux du secteur de l'éducation .........................................................46

Encadré 11. Les versions « light » de l'Ed-DQAF et du CdP, conçues par l'ISU pour les systèmes de données administratives courants 


\section{Acronymes et abréviations}

ACER Conseil australien de recherche en éducation - Centre pour le suivi mondial de l'éducation

BREDA Bureau régional pour l'Éducation en Afrique (aujourd'hui, Bureau régional de l'UNESCO à Dakar)

CapED Programme de renforcement des capacités en faveur de l'éducation de l'UNESCO

CCS-UNS Comité des statisticiens en chef du système des Nations Unies

CdP Code de pratiques

CEPALC Commission économique pour l'Amérique latine et les Caraïbes

CITÉ Classification internationale de type de l'éducation

CSA-CEPALC Conférence statistique des Amériques de la Commission économique pour l'Amérique latine et les Caraïbes

CSNU Commission de statistique des Nations Unies

DANE Departamento Administrativo National de Estadística (Département national de la statistique)

DHS Enquête démographique et de santé

DFAT Ministère des Affaires étrangères et du commerce (Australie)

DQAF Cadre d'assurance de la qualité des données

DSNU Division de statistique des Nations Unies

ECOSOC Conseil économique et social des Nations Unies

ED 2030 SC Comité directeur de l'agenda Éducation 2030

Ed-DQAF Cadre d'assurance de la qualité des données de l'éducation

EPT Éducation pour tous

FAO Organisation des Nations Unies pour l'alimentation et l'agriculture

FMI Fonds monétaire international

GAML l'Alliance mondiale pour le suivi de l'apprentissage

GCT Groupe consultatif technique sur les indicateurs de l'éducation post-2015

GdCT Groupe de coopération technique sur les indicateurs du Cadre d'action ODD 4-Éducation 2030

GEM Report Global Education Monitoring Report

GIAE-ODD Groupe inter-agences et d'experts sur les indicateurs des ODD

GPE Partenariat mondial pour l'éducation

GP-LA Principes des bonnes pratiques dans l'évaluation des apprentissages

GT-CVD Groupe de travail sur la communication, la validation et la diffusion des données

GT-EI Groupe de travail sur l'élaboration d'indicateurs

GT-RCS Groupe de travail sur le renforcement des capacités statistiques

HHS enquête auprès des ménages

HLG-PCCB Groupe de haut niveau pour le partenariat, la coordination et le renforcement des capacités pour le suivi du programme de développement à l'horizon 2030

HLPF Forum politique de haut niveau des Nations unies sur le développement durable

IPUMS Integrated Public Use Microdata Series-International 
ISU Institut de statistique de I'UNESCO

LaNA l'Évaluation des capacités en lecture, écriture et calcul

MDG Objectif du millénaire pour le développement

MICS I'Enquête par grappes à indicateurs multiples

NEGED Groupe d'experts nationaux des données de l'éducation

NIF Cadre d'indicateurs national

NQAF Cadre national d'assurance de la qualité

OCDE Organisation de coopération et de développement économiques

ODD Objectif de développement durable

ONU Organisation des Nations Unies

OSC Organisation de la société civile

Paris21 Partenariat en statistique pour le développement au $21^{\text {ème }}$ siècle

PASEC Programme d'analyse des systèmes éducatifs de la CONFEMEN

PILNA Évaluation des capacités en lecture, écriture et calcul des îles du Pacifique

PIRLS Programme international de recherche en lecture scolaire

PISA Programme PISA

PNB Produit national brut

QAF $\quad$ Cadre d'assurance qualité

SACMEQ Consortium d'Afrique australe pour le pilotage de la qualité de l'éducation

SADC Communauté de développement d'Afrique australe

SDMX Échange de données et de métadonnées statistiques

SEA PLM Principales métriques d'apprentissage en Asie du Sud-Est

SIGE Système d'information et de gestion de l'éducation

SNDSE Stratégie nationale de développement des statistiques de l'éducation

SNDS Stratégies nationales de développement des statistiques

SSNE Système statistique national de l'éducation

SSE Système statistique européen

TERCE Tercer Estudio Regional Comparativo y Explicativo

TIC technologies de l'information et de la communication

TIMSS Tendances dans les études internationales de mathématiques et de sciences

PNUD Programme des Nations Unies pour le développement

UE Union européenne

UNDESA Département des affaires économiques et sociales des Nations Unies

UNESCO Organisation des Nations Unies pour l'éducation, la science et la culture

UNGA Assemblée générale des Nations Unies

UNICEF Fonds des Nations Unies pour l'enfance

UN SQAF Cadre d'assurance de la qualité des statistiques 


\section{Avant-propos}

Les objectifs de développement durable (ODD) et le Cadre d'action Éducation 2030 définissent un ordre du jour ambitieux visant à ce que les pays donnent accès à une éducation de qualité et à des résultats d'apprentissage efficaces pour tous. Pour l'ensemble des objectifs, la priorité accordée à l'équité cherche à s'assurer que personne n'est laissé sur le bord de la route, en particulier les groupes les plus pauvres et les plus vulnérables. En moins de 13 ans, la communauté internationale doit transformer cet engagement décisif en réalité pour l'ensemble des enfants et des jeunes, quel que soit le lieu où ils vivent ou la situation à laquelle ils sont confrontés.

L'expérience du précédent programme relatif à l'éducation confirme que la dure réalité de la mise en œuvre atrophie les ambitions. Alors que l'accès à l'éducation primaire a globalement progressé depuis 2000 , des améliorations significatives des opportunités équitables et de la qualité de l'apprentissage sont encore justifiées. L'une des leçons importantes qui ont été tirées est la nécessité de disposer de mesures quantitatives valables pour surveiller l'élaboration et la mise en œuvre des politiques d'éducation aux niveaux national et international. L'augmentation du nombre de cibles et d'indicateurs de l'ODD 4-Cadre d'action Éducation 2030 souligne l'importance du suivi de l'éducation au plan international.

Le nouveau programme relatif à l'éducation exige des pays un effort sans précédent pour qu'ils produisent des données en plus grande quantité et de meilleure qualité. Des efforts visant à créer des systèmes statistiques nationaux plus solides et plus fiables sont déjà déployés dans de nombreux pays, mais les nouveaux indicateurs et le niveau de détail requis engendrent des défis considérables au niveau national. II existe un besoin urgent d'améliorer la qualité des données qui sont nécessaires pour surveiller et atteindre l'ODD4Cadre d'action Éducation 2030.

Le présent rapport, Le facteur qualité : Renforcer les données nationales afin de surveiller l'Objectif de développement durable 4, est le second dans la série Rapport sur les données du développement durable. Ce volume propose un cadre conceptuel et un ensemble concret d'outils qui vont aider les pays à améliorer la qualité de leurs données et à répondre à leurs exigences en matière de présentation de rapports.

Le rapport précédent, Jeter les bases pour mesurer l'objectif de développement durable 4, explique comment les pays et les spécialistes de l'éducation se sont rapprochés pour élaborer un accord global pour l'éducation, mesuré grâce à une liste de 43 indicateurs mondiaux et thématiques. En ce qui concerne la qualité des données, le Rapport sur les données du développement durable 2016 diffusait deux mises en garde. Premièrement, en dépit de décennies de coopération internationale pour surveiller l'éducation, de nombreux pays n'étaient pas préparés de façon appropriée à livrer les données, soit parce que les indicateurs relatifs à l'ODD 4 n'existaient pas encore ou parce qu'elles ne pouvaient pas être produites d'une façon fiable et régulière. Deuxièmement, la qualité et la comparabilité au plan international des indicateurs de suivi dépendent directement de la qualité des données nationales relatives à l'éducation, en particulier lorsqu'il s'agit de faire face aux complexités des différents types de sources de données.

Le rapport de cette année s'attaque directement à ces problèmes en présentant les derniers efforts déployés par les groupes de travail internationaux et l'Institut de statistique de l'UNESCO (ISU) pour aider les pays à mettre en œuvre les cadres des indicateurs mondiaux et thématiques. Le Rapport sur les données du développement durable montre comment les travaux de l'Institut pour renforcer les systèmes statistiques nationaux relatifs à l'éducation jettent également les bases de la comparabilité transnationale nécessaire pour surveiller les progrès réalisés au plan mondial. S'appuyant sur les résultats d'une série d'évaluations diagnostiques de la qualité et de la disponibilité des données, l'ISU aide les pays à élaborer leurs propres stratégies nationales et à améliorer leurs systèmes statistiques nationaux, tout en renforçant les capacités techniques de leurs parties prenantes nationales. Au niveau international, I'ISU continue à innover dans le cadre de son mandat afin d'élaborer les méthodologies et les normes nécessaires pour les nouveaux indicateurs. 
Ces travaux s'inscrivent tous dans la vision que s'est forgée l'ISU, soit une révolution des données sur l'éducation qui repose sur trois piliers et qui implique des acteurs clés - à savoir les gouvernements, les partenaires du développement et I'ISU. Premièrement, nous devons créer des environnements favorables au sein desquels les communautés, les gouvernements et la société civile peuvent utiliser les données relatives à l'éducation pour mener des actions et favoriser la responsabilité. Le second pilier concerne la production de données de haute qualité comparables au plan transnational, et passe par le soutien des travaux de l'ISU en matière de définition des méthodologies et d'élaboration des normes, ainsi que de ses activités de collecte et de production des données. Le troisième pilier s'appuie sur les efforts en cours pour renforcer la diffusion et l'utilisation des données par les gouvernements, communautés et groupes de la société civile.

Comment pouvons-nous construire ces piliers ? Il y a un besoin urgent de financement et d'engagement durables afin que les données sur l'éducation occupent la place qui leur est due en tant que biens publics et outils d'élaboration de politiques. Au niveau national, les gouvernements et les donateurs doivent mieux soutenir les capacités des pays de produire, diffuser et utiliser les données relatives à l'éducation.

Il est par conséquent essentiel d'élargir le champ des ressources en exploitant de nouvelles sources de financement, en particulier dans le secteur privé. Par exemple, selon le document de I'ISU, "La révolution des données sur l'éducation » (ISU, 2017), il suffirait de seulement $0,002 \%$ des recettes annuelles totales des 14 plus grandes sociétés du secteur des technologies de l'information pour équiper les pays des technologies de base dont ils ont besoin pour collecter, traiter et diffuser les données relatives à l'éducation.

La communauté internationale a clairement pris acte du pouvoir transformateur des données pour atteindre les objectifs de développement. Les nouveaux indicateurs de l'ODD 4 sont considérés comme des investissements permettant d'assurer une formulation efficace des politiques et des actions. Les coûts initiaux d'élaboration des outils statistiques et le soutien dont les pays ont besoin sont dérisoires par rapport aux économies que ces outils permettraient de réaliser en matière de budgets nationaux de l'éducation et d'aide étrangère. Cependant, les véritables bienfaits des données sont clairement perçus lorsque nous imaginons tous les enfants et les jeunes à l'école et en train d'apprendre.

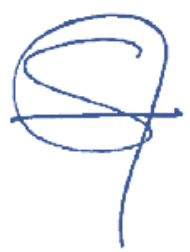

Silvia Montoya

Directrice 


\section{Introduction}

Le nouveau Programme de développement durable à l'horizon 2030 et son cadre d'indicateurs fixent des normes exigeantes pour les systèmes d'information nationaux et internationaux. Surveiller la mise en œuvre et les progrès des multiples composants de l'objectif distinct relatif à l'éducation (objectif de développement durable [ODD] 4) exige des données de qualité. Définir les normes de la production de données de qualité et évaluer la qualité des données à cette fin sont les défis qui sous-tendent les mécanismes de suivi nationaux et internationaux déjà en place. En particulier, quels changements doivent être effectués et qui doit les mettre en œuvre sont des questions fondamentales auxquelles il faut répondre pour obtenir des données sur l'éducation viables et comparables au plan transnational.

Pendant le processus d'élaboration des indicateurs et de production des données, les pays et les groupes consultatifs ont prôné la transparence et la documentation appropriée des processus statistiques. L'Institut de statistique de l'UNESCO (ISU) et ses agences partenaires sont des contributeurs majeurs - en termes de collecte des données, d'élaboration des indicateurs et de renforcement des systèmes de données nationaux d'un suivi de la qualité de l'ODD 4. Compte tenu de l'écart qui existe entre les capacités nationales en matière de statistiques et l'ensemble des attentes, considérables, relatives à la production de données de qualité, I'ISU fournit aux parties prenantes nationales les méthodologies et les instruments dont elles ont besoin pour relever le défi du suivi de l'ODD 4.

L'édition de cette année du Rapport sur les données du développement durable est consacrée au thème suivant : "Données de qualité pour surveiller les progrès réalisés sur la voie de l'ODD 4 et développement des capacités des pays en matière de statistiques ". La Section 1 examine le nouveau cadre de suivi de l'ODD 4 et définit les niveaux mondiaux et thématiques du suivi de l'éducation. Elle informe le lecteur des événements et processus récents qui ont mené à l'élaboration d'une sélection proposée de 43 indicateurs - 11 indicateurs mondiaux et 32 indicateurs thématiques - qui permettront de surveiller l'ODD 4. À l'intérieur de ce cadre, il est nécessaire d'élaborer et de valider de nouvelles méthodologies et d'affiner les méthodologies existantes afin d'assurer la comparabilité transnationale. Dans l'état actuel des choses, de nombreux pays auront du mal à satisfaire aux exigences en matière d'établissement des rapports. Un urgent besoin de renforcement des capacités de collecte des données nationales nécessaires au suivi de l'ODD 4 se fera sentir.

La Section 2 décrit l'approche de l'ISU en matière d'amélioration de la qualité des données sur l'éducation aux niveaux national et international. S'appuyant sur l'appel en faveur d'une révolution des données, les outils de renforcement des capacités de l'ISU peuvent permettre aux pays d'améliorer la qualité de leurs systèmes nationaux de statistiques de l'éducation. Les cadres d'évaluation de la qualité des données utilisés au niveau de la planification nationale et au niveau du processus statistique servent d'outils de diagnostic. Les efforts durables et coordonnés entre les parties prenantes régionales et internationales sont également importants pour définir et valider des données sur l'éducation comparables.

La Section 3 s'intéresse aux activités récemment menées par l'ISU au niveau national pour construire de meilleurs systèmes statistiques et impliquer les acteurs nationaux dans l'appropriation et la durabilité de leurs processus. L'ISU travaille avec des systèmes statistiques nationaux afin de tenir compte des besoins en matière de données sur l'éducation et d'élaborer une approche des statistiques de l'éducation pour l'ensemble du secteur, dans le but d'améliorer la qualité des indicateurs de l'ODD 4. Pour mettre en œuvre cette approche, l'ISU a élaboré un ensemble d'outils qui sont adaptés à une évaluation de la disponibilité des données essentielles par les équipes nationales et qui permettent d'évaluer la qualité des environnements institutionnels, des processus statistiques et des résultats statistiques pour chacun des systèmes d'information existants dans le domaine de l'éducation. 


\section{Suivre l'agenda international de l'éducation}

En septembre 2015, les 193 États membres des Nations Unies (ONU) ont unanimement adopté le Programme de développement durable à l'horizon 2030. S'appuyant sur les objectifs de développement durable (ODD) approuvés en 2000, l'Agenda 2030 comprend 17 objectifs de développement durable (ODD) et 169 cibles. Les États membres se sont engagés autour d'un agenda mondial partagé afin de faire disparaître la pauvreté et les inégalités généralisées en améliorant les droits sociaux et humains, en promouvant une croissance économique durable et en protégeant l'environnement (ONU, 2000, 2015). Le cadre d'indicateurs des ODD associé à l'Agenda a été élaboré par le Groupe interagences et d'experts sur les indicateurs des ODD (GIAE-ODD), un groupe créé par la Commission de statistique des Nations Unies, et adopté par l'Assemblée générale de l'ONU en juillet 2017.

L'éducation est un thème central de l'Agenda 2030, qui comprend un objectif sur l'éducation distinct ainsi que des cibles liées à l'éducation dans sept autres ODD (ISU, 2016) ${ }^{1}$. En particulier, l'ODD 4 est ambitieux et cherche à "Assurer l'accès de tous à une éducation de qualité, sur un pied d'égalité, et promouvoir les possibilités d'apprentissage tout au long de la vie » d'ici à 2030. L'objectif comprend dix cibles visant à guider les pays tout au long de la voie transformatrice conduisant à un agenda de l'éducation durable.

L'ODD 4 a une optique plus large que l'Objectif 2, lié à l'éducation, des OMD ("Universaliser l'accès à l'enseignement primaire ») et va au-delà des objectifs de l'Éducation pour tous (EPT) adoptés par la communauté internationale de l'éducation, à Dakar, en $2000^{2}$. L'ODD 4 est global car il couvre les sous-secteurs, de l'éducation préscolaire à l'apprentissage tout au long de la vie. La qualité de l'apprentissage, l'inclusion et l'équité sont des éléments fondamentaux de la réalisation de l'objectif. Au-delà de l'éducation, I'ODD 4 est également lié à d'autres objectifs, en termes d'acquisition de connaissances et de compétences, visant à promouvoir le développement durable, à éliminer les disparités entre les sexes et à élargir l'accès à l'éducation à tous les jeunes et à tous les adultes de façon à améliorer leurs possibilités d'emploi.

Depuis septembre 2015, plusieurs processus ont commencé - ou ont continué, dans certains cas afin d'amorcer la mise en œuvre d'un cadre de suivi coordonné et intégré et l'élaboration d'indicateurs pour surveiller l'ODD 4. La première édition du Rapport sur les données du développement durable (2016) a documenté ces efforts, notamment la sélection des critères de suivi et la définition des principaux indicateurs et cibles par le biais d'un processus conduit au niveau des pays et guidé par des experts et des groupes consultatifs. Cette section met à jour la discussion du Rapport 2016 sur les activités liées à l'élaboration des processus de suivi et des définitions d'indicateurs de l'ODD 4.

\subsection{Le nouveau cadre de suivi de I'ODD 4}

L'examen de la mise en œuvre du cadre de suivi des OMD depuis 2000 a permis de tirer des enseignements pour l'élaboration des critères de suivi des ODD. D'un point de vue statistique, le cadre des OMD reposait sur un ensemble d'indicateurs concrets et quantifiables et a généralement favorisé, dans plusieurs pays en développement, l'amélioration des capacités nationales en matière de suivi statistique. Néanmoins, certains défis liés aux données ont été révélés, notamment l'absence de clarté ou des incohérences entre les objectifs et les indicateurs, ainsi que l'insuffisance du soutien technique et financier pour améliorer les systèmes de suivi nationaux. Le lien entre les indicateurs nationaux et mondiaux était confus pour certains objectifs, des indicateurs ne tenaient pas suffisamment compte des inégalités entre les groupes, et la qualité des données variait à cause d'écarts et de différences

\footnotetext{
${ }^{1}$ Les autres cibles liées à l'éducation sont incluses dans l'ODD 1 (sur la pauvreté), l'ODD 3 (sur la santé et le bien-être), I'ODD 5 (sur l'égalité des sexes), I'ODD 8 (sur le travail décent et la croissance économique), l'ODD 12 (sur la consommation et la production responsables), l'ODD 13 (sur le changement climatique) et l'ODD 16 (sur la paix, la justice et la solidité des institutions). Voir le Tableau 2 dans ISU, 2016.

${ }^{2}$ Pour en savoir plus sur les cibles et indicateurs liés à l'éducation, voir la Déclaration du Millénaire de l'Assemblée générale de l'ONU et le Cadre d'action de Dakar (ONU, 2000 ; UNESCO, 2000).
} 
entre les fournisseurs de données (GIAE-OMD, 2013).

En décembre 2014, le Secrétaire général de l'ONU, Ban Ki Moon, a préconisé une approche complète du suivi des ODD, qui a ensuite trouvé un écho dans l'appel à l'action collectif et universel de l'Agenda 2030 (ONU, 2015 ; SGONU, 2014). Tout en soulignant l'importance de l'efficacité, de l'efficience, des preuves et de l'universalité en tant que principes directeurs de l'examen des progrès des ODD, le Secrétaire général de l'ONU a également recommandé d'utiliser un cadre participatif grâce auquel l'ensemble des parties prenantes et des groupes concernés (par exemple, la société civile, les entreprises, le parlement, l'université et le gouvernement) pourront reconnaître leur responsabilité partagée dans la réalisation des ODD. La Figure 1 montre ce cadre polyvalent, à paliers multiples, composé de quatre niveaux de suivi - national, régional, mondial et thématique ayant les caractéristiques suivantes.

\section{- Le suivi au niveau national de l'ODD 4 est} lié aux besoins des gouvernements nationaux et infranationaux en matière d'élaboration des plans relatifs au secteur de l'éducation et d'enrichissement des politiques d'éducation. Les données qui offrent la granularité la plus fine et qui s'adaptent aux spécificités du contexte national - comme dans les unités géographiques infranationales, les groupes désavantagés spécifiques ou par niveau de revenu - sont davantage en mesure d'éclairer les politiques, notamment en mettant en lumière les disparités pertinentes dans les résultats d'éducation. Le suivi de l'ODD 4 à ce niveau bénéficie de la participation active d'un groupe varié de parties prenantes représentant leurs partenaires respectifs et leurs préoccupations en matière d'éducation.

- Au niveau régional de suivi, un ensemble d'indicateurs peuvent être élaborés pour prendre en considération les priorités et les problèmes d'intérêt commun qui sont partagés par les pays d'une région particulière, comme le soulignent les documents de planification ou les cadres régionaux. Certains cadres ont pour but de surveiller spécifiquement les ODD dans le contexte d'une politique régionale. C'est le cas de l'Ensemble d'indicateurs des ODD de I'Union européenne (UE), qui est composé de 100 indicateurs permettant d'effectuer le suivi de 17 ODD. Les six indicateurs régionaux sélectionnés pour surveiller l'ODD 4 ont des liens étroits avec la cadre stratégique Éducation et formation 2020 de l'UE, centré sur l'investissement en faveur des jeunes et l'augmentation des possibilités d'apprentissage tout au long de la vie (Eurostat, 2017). Dans un esprit différent, l'Union africaine a élaboré un ensemble ambitieux d'objectifs de développement pour la région dans le Documentcadre Agenda 2063 : l'Afrique que nous voulons,

Figure 1. Les quatre niveaux du suivi des cibles liées à l'éducation

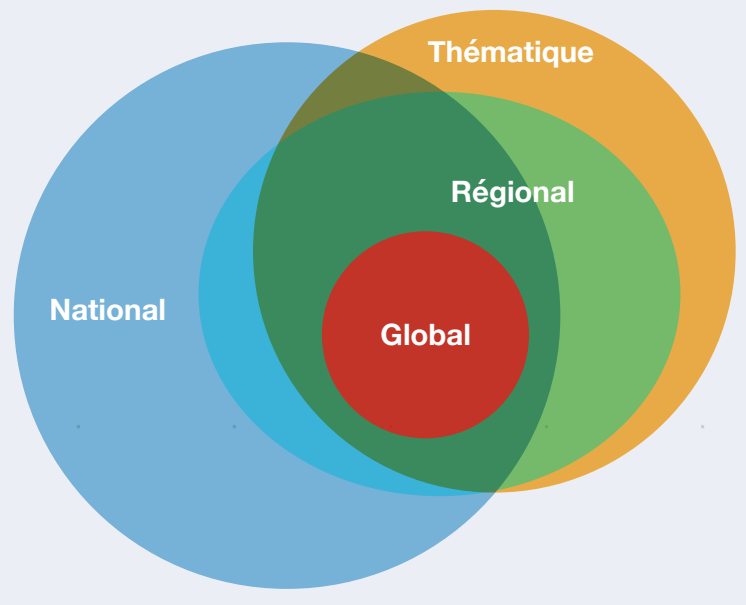

Source : Institut de statistique de I'UNESCO, 2016. 
qui comprend son propre ensemble d'indicateurs spécifiques à la région. Intégrer les systèmes de suivi de l'Agenda 2063 et des ODD fait partie d'un plan de mise en œuvre de dix ans à l'intention des gouvernements nationaux (Commission de l'Union africaine, 2015a, 2015b).

- Le suivi au niveau mondial repose sur un groupe plus restreint d'indicateurs majeurs, soigneusement sélectionnés pour offrir une vue d'ensemble des progrès réalisés dans l'atteinte de chacune des cibles. L'harmonisation du suivi et de la présentation des rapports relatifs aux ODD est également d'une importance capitale. La capacité à analyser et à comparer les données nationales d'un pays ou d'une année à l'autre permet d'obtenir des renseignements précieux sur la qualité des mesures, d'orienter les réformes des politiques et d'affecter les ressources équitablement pour améliorer l'apprentissage dans l'ensemble des groupes de population. Le partage des connaissances et l'examen universel sont chaque année à l'ordre du jour du Forum politique de haut niveau des Nations Unies sur le développement durable (HLPF) (ONU, 2017).

- Le suivi thématique ajoute un niveau de suivi d'indicateurs comparables dans un secteur spécifique (par exemple, l'éducation, l'environnement, l'énergie, la santé) ou pour un thème transversal (par exemple, le sexe). Les indicateurs thématiques servent de cadre pour suivre les progrès réalisés sur une base comparable au plan transnational, et offrent un point de vue plus approfondi des priorités sectorielles que le cadre de suivi mondial. Grâce à l'intervention du Secrétaire général, ce niveau donne l'occasion d'identifier des défis et des obstacles spécifiques à des secteurs et de mobiliser l'action requise pour les traiter. Le HLPF organise chaque année des réunions thématiques où sont réalisés des examens approfondis de problèmes transversaux, tels que l'élimination de la pauvreté (en 2017), la durabilité et la résilience (en 2018) et l'autonomisation, l'inclusion et l'égalité (en 2019) (UNDESA, 2017).

Le 4 novembre 2015, les 184 États membres de I'UNESCO ont adopté le Cadre d'action Éducation 2030, qui offre des orientations (" stratégies indicatives »), aux plans national, régional et mondial, pour atteindre l'ODD 4 et suivre la mise en œuvre de chacune des dix cibles de l'ODD4. Le Cadre d'action Éducation 2030 souligne que le suivi de l'ODD 4 requiert " une approche multidimensionnelle, qui englobe la phase de conception, les éléments d'appui, le contenu, les processus et les résultats du système » (UNESCO, 2015, par. 97). II désigne également l'Institut de statistique de I'UNESCO comme source officielle des données sur l'éducation comparables entre les pays et demande au Rapport mondial de suivi sur l'Éducation (RMSE) de surveiller et d'évaluer les progrès réalisés sur la voie de l'ODD 4 et des autres objectifs liés à l'éducation ${ }^{3}$. Les gouvernements nationaux ont la responsabilité fondamentale de construire des mécanismes de suivi conformes au consensus obtenu aux plans régional et mondial et en consultation avec les organisations de la société civile (OSC). Le document est le résultat de 18 mois d'efforts collectifs caractérisés par les nombreuses consultations régionales et nationales conduites par les gouvernements et la société civile. L'UNESCO et les autres partenaires internationaux ont facilité ce processus (UNESCO, 2015).

Le Cadre d'action Éducation 2030 comprend un projet de liste de 43 indicateurs thématiques $-y$ compris 11 indicateurs mondiaux - visant à cartographier les progrès mondiaux en matière d'éducation. L'ISU, avec l'apport des organisations partenaires et des experts des États membres et de la société civile, a élaboré cette proposition afin de fournir aux pays des orientations de suivi s'articulant autour de concepts liés à l'éducation en rapport avec les cibles mondiales. L'amélioration constante et le développement de ces indicateurs dans les cadres de surveillance s'inscrivent dans plusieurs processus qui sont décrits dans la section suivante.

\subsection{L'élaboration et la mise en œuvre des cadres d'indicateurs mondiaux et thématiques de I'ODD 4}

Comme pour les OMD, les progrès réalisés sur la voie de chacun des ODD doivent être régulièrement surveillés entre aujourd'hui et 2030. Sélectionner et définir des indicateurs pour surveiller les cibles mondiales en matière d'éducation est un processus qui s'est adossé à l'expérience des OMD. Évaluer les progrès sur la voie d'un objectif

${ }^{3}$ Lancé en 2016, le RMSE était précédemment intitulé Rapport mondial de suivi sur l'Éducation pour tous. 
international exige de disposer d'instruments de mesure pertinents pour analyser l'impact des politiques d'éducation nationales et pour parvenir à un ensemble d'indicateurs comparables au plan international.

\subsubsection{Le cadre des indicateurs mondiaux}

En décembre 2014, la Commission de statistique de l'ONU (CSNU) a créé le Groupe interagences et d'experts sur les indicateurs des ODD (GIAEODD), composé du Président de la CSNU et de 27 experts d'instituts de statistiques nationaux représentatifs des régions, avec pour mandat d'élaborer un cadre d'indicateurs mondial pour l'ensemble des ODD ${ }^{4}$. Au terme de plusieurs séries de consultations et de réunions mondiales avec les États membres de l'ONU, des organisations régionales et internationales, des universités, des entreprises, des ONG et la société civile, le GIAEODD a initialement proposé, en mars 2016, une liste de 11 indicateurs mondiaux sur l'éducation visant à surveiller l'ODD 4, qui a finalement été approuvée lors de la $48^{\mathrm{e}}$ session de la CSNU, en mars 2017, et officiellement adoptée par le Conseil économique et social des Nations Unies (ECOSOC) et l'Assemblée générale de l'ONU en juin et juillet 2017 , respectivement ${ }^{5}$. Ce cadre de suivi mondial de l'ODD 4 constitue l'ensemble d'indicateurs de base considéré comme indispensable pour que les pays puissent surveiller l'objectif en matière d'éducation (voir Tableau 1).

Lorsque le consensus a été atteint sur la liste d'indicateurs mondiaux de l'éducation, le GIAEODD a élaboré un outil de classification par niveaux afin d'identifier les phases d'élaboration de chacun des indicateurs ainsi que leur disponibilité à l'échelle mondiale (voir Encadré 1). Les indicateurs de Niveau 1 et de Niveau 2 sont associés à des méthodologies et à des normes établies au plan international, mais les indicateurs de Niveau 2 ne sont pas régulièrement produits par les pays. Les indicateurs de Niveau 3 exigent l'élaboration de méthodologies et de normes, et ce travail est devenu l'une des priorités du GIAE-ODD. Tous les indicateurs sont considérés comme aussi importants les uns que les autres pour surveiller l'ODD 4, indépendamment de leur niveau dans la classification.

L'élaboration et la validation des nouvelles méthodologies propres aux indicateurs mondiaux de Niveau 3 relèvent de la responsabilité des organismes dépositaires et partenaires de l'indicateur. Le GIAE-ODD, qui supervise ce processus, a identifié trois organismes dépositaires pour les indicateurs mondiaux de l'éducation. L'ISU est un organisme dépositaire pour 9 des 11 indicateurs mondiaux de I'ODD 4 et un organisme partenaire pour les deux autres. L'UNICEF et l'Organisation de coopération et de développement économiques (OCDE) sont les deux autres organismes dépositaires pour les indicateurs mondiaux suivants : un indicateur lié au développement de l'enfant (4.2.1) et un indicateur lié à l'aide publique au développement consacrée aux bourses d'études (4.b.1)

Le GIAE-ODD organise deux réunions annuelles dans le cadre desquelles il examine la liste des indicateurs et étudie les modifications ou améliorations mineures de ses membres ou les clarifications éditoriales de la Division de statistique des Nations Unies (DSNU) ${ }^{7}$. Pendant ces réunions, le GIAE-ODD évalue également la classification en niveaux pour un groupe d'indicateurs sélectionnés, examine les méthodologies proposées par les organismes dépositaires et donne son approbation finale pour les modifications apportées à la classification en niveaux. Sur la base des résultats de ces réunions annuelles, le GIAE-ODD émet des recommandations afin que la CSNU approuve les améliorations.

Outre les examens annuels, le GIAE-ODD conduira des examens quinquennaux du cadre des indicateurs mondiaux en 2019 et en 2024 afin de préparer les sessions d'examen de la CSNU de 2020 et 2025. Les modifications importantes ne sont étudiées

\footnotetext{
${ }^{4}$ Pour les membres, voir l'Encadré 3 de la Section 1.2 du Rapport sur les données du développement durable (2016) (ISU, 2016).

${ }^{5}$ Les indicateurs mondiaux de l'ODD 4 ont été élaborés par le GIAE-ODD dans le cadre d'un processus d'élaboration d'indicateurs pour d'autres 0DD.

${ }^{6}$ Certains indicateurs liés à l'éducation sont suivis dans le cadre d'autres Objectifs, notamment les ODD 1, 8, 12, 13 et 16 . Les informations sur le statut de ces indicateurs sont disponibles sur le site Web du GIAE-ODD : https://unstats.un.org/ODDs/iaeg-ODDs/

${ }^{7}$ Les améliorations peuvent comprendre les types suivants de modifications : «spécifier ou corriger l'unité de mesure ; simple clarification des termes utilisés dans l'indicateur ; orthographe ou autres erreurs évidentes ; ou "fractionner" les indicateurs dans leurs composants dans des indicateurs à composants multiples. Une amélioration peut également consister à apporter une modification mineure dans un indicateur ou dans une liste d'indicateurs qui, d'une façon simple, résout un problème qui est décelé lorsque débute la collecte des données » (GIAE-0DD, 2016, p. 2).
} 
Tableau 1. Statut actuel des indicateurs mondiaux et thématiques du suivi de I'ODD 4

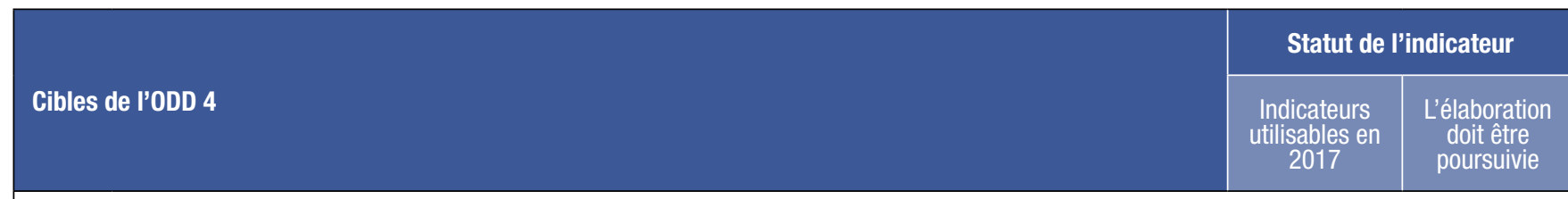

Cible 4.1 D'ici à 2030, faire en sorte que toutes les filles et tous les garçons suivent, sur un pied d'égalité, un cycle complet d'enseignement primaire et secondaire gratuit et de qualité, les dotant d'acquis véritablement utiles.

\begin{tabular}{|c|c|c|c|}
\hline 4.1.1 & $\begin{array}{l}\text { Proportion d'enfants et de jeunes (a) en } 2 \text { e ou } 3 \text { e année ; (b) en fin de cycle primaire ; (c) en fin de } \\
\text { premier cycle du secondaire qui maîtrisent au moins les normes d'aptitudes minimales en (i) lecture et } \\
\text { (ii) mathématiques, par sexe. }\end{array}$ & $\mathrm{x}$ & $\mathrm{X}$ \\
\hline 4.1 .2 & $\begin{array}{l}\text { Administration d'une évaluation de l'apprentissage représentative au plan national (a) en 2e ou 3e année ; } \\
\text { (b) en fin de cycle primaire ; et (c) en fin de premier cycle du secondaire }\end{array}$ & $\mathrm{x}$ & \\
\hline 4.1.3 & Taux brut de scolarisation jusqu'au dernier niveau (primaire, premier cycle du secondaire) & $X$ & \\
\hline 4.1.4 & Taux d'achèvement (primaire, premier cycle du secondaire et second cycle du secondaire) & $\mathrm{X}$ & \\
\hline 4.1.5 & Taux d'enfants non scolarisés (cycle primaire, premier cycle du secondaire et second cycle du secondaire) & $\mathrm{X}$ & \\
\hline 4.1.6 & $\begin{array}{l}\text { Pourcentage des enfants ayant dépassé l'âge normal d'un niveau (cycle primaire, premier cycle du } \\
\text { secondaire) }\end{array}$ & $\mathrm{X}$ & \\
\hline 4.1.7 & $\begin{array}{l}\text { Nombre d'années d'enseignement primaire et secondaire (i) gratuit et (ii) obligatoire garanti par le cadre } \\
\text { juridique }\end{array}$ & $\mathrm{X}$ & \\
\hline \multicolumn{4}{|c|}{$\begin{array}{l}\text { Cible 4.2 D’ici à 2030, faire en sorte que toutes les filles et tous les garçons aient accès à des activités de développement et de soins de la } \\
\text { petite enfance et à une éducation préscolaire de qualité qui les préparent à suivre un enseignement primaire }\end{array}$} \\
\hline 4.2.1 & $\begin{array}{l}\text { Pourcentage d'enfants de moins de cinq ans dont le développement est en bonne voie en matière de santé, } \\
\text { d'apprentissage et de bien-être psychosocial, par sexe }\end{array}$ & $\mathrm{X}$ & $\mathrm{X}$ \\
\hline 4.2 .2 & $\begin{array}{l}\text { Taux de participation à des activités organisées d'apprentissage (un an avant l'âge officiel de scolarisation } \\
\text { dans le primaire), par sexe }\end{array}$ & $\mathrm{x}$ & \\
\hline 4.2 .3 & $\begin{array}{l}\text { Pourcentage d'enfants de moins de cinq ans vivant dans un environnement d'apprentissage positif et } \\
\text { stimulant à la maison }\end{array}$ & & $\mathrm{x}$ \\
\hline 4.2 .4 & $\begin{array}{l}\text { Taux brut de scolarisation préscolaire dans (a) l'enseignement préscolaire et (b) les activités de } \\
\text { développement de la petite enfance }\end{array}$ & $\mathrm{x}$ & \\
\hline 4.2 .5 & Nombre d'années d'enseignement préscolaire (i) gratuit et (ii) obligatoire garanti par le cadre juridique & $x$ & \\
\hline \multicolumn{4}{|c|}{$\begin{array}{l}\text { Cible 4.3 D'ici à 2030, faire en sorte que les femmes et les hommes aient tous accès dans des conditions d'égalité à un enseignement } \\
\text { technique, professionnel ou tertiaire, y compris universitaire, de qualité et d'un coût abordable }\end{array}$} \\
\hline 4.3 .1 & $\begin{array}{l}\text { Pourcentage de jeunes et d'adultes ayant participé à un programme d'éducation et de formation formelle } \\
\text { ou non formelle au cours des } 12 \text { derniers mois, par sexe }\end{array}$ & $x$ & $\mathrm{X}$ \\
\hline 4.3 .2 & Taux brut de scolarisation dans l'enseignement supérieur, par sexe & $\mathrm{X}$ & \\
\hline 4.3.3 & Taux de participation aux programmes d'enseignement technique et professionnel (15-24 ans), par sexe & $\mathrm{x}$ & \\
\hline
\end{tabular}


Cible 4.4 D'ici à 2030, augmenter considérablement le nombre de jeunes et d'adultes disposant des compétences, notamment techniques et professionnelles, nécessaires à l'emploi, à l'obtention d'un travail décent et à l'entrepreneuriat

\begin{tabular}{|c|l|c|c|}
\hline 4.4 .1 & $\begin{array}{l}\text { Pourcentage de jeunes et d'adultes ayant des compétences en matière de technologies de l'information et } \\
\text { de la communication (TIC), par type de compétence }\end{array}$ & $X$ & $X$ \\
\hline 4.4 .2 & $\begin{array}{l}\text { Pourcentage de jeunes et d'adultes qui ont acquis au moins un niveau minimum de compétences en } \\
\text { matière d'alphabétisation numérique }\end{array}$ & $X$ \\
\hline 4.4 .3 & $\begin{array}{l}\text { Taux de réussite scolaire des jeunes et des adultes par classe d'âge, situation au regard de l'activité } \\
\text { économique, niveau d'études et orientation scolaire }\end{array}$ & $X$ & $\begin{array}{c}X \\
\text { (à simplifier) }\end{array}$ \\
\hline
\end{tabular}

Cible 4.5 D'ici à 2030, éliminer les inégalités entre les sexes dans le domaine de l'éducation et assurer l'égalité d'accès des personnes vulnérables, y compris les personnes handicapées, les autochtones et les enfants en situation vulnérable, à tous les niveaux d'enseignement et de formation professionnelle

\begin{tabular}{|c|c|c|c|}
\hline 4.5 .1 & $\begin{array}{l}\text { Indices de parité (femmes/hommes, urbain/rural, quintile inférieur/supérieur de richesse et autres } \\
\text { paramètres tels que le handicap, le statut d'autochtone et les situations de conflit, à mesure que les } \\
\text { données deviennent disponibles) pour tous les indicateurs de l'éducation de cette liste pouvant être ventilés }\end{array}$ & $\mathrm{x}$ & \\
\hline 4.5 .2 & $\begin{array}{l}\text { Pourcentage d'élèves de l'enseignement primaire dont la première langue ou la langue maternelle est la } \\
\text { langue d'instruction }\end{array}$ & & $\mathrm{X}$ \\
\hline 4.5 .3 & $\begin{array}{l}\text { Mesure dans laquelle les stratégies explicITÉs basées sur une formule réaffectent les ressources de } \\
\text { l'éducation aux populations défavorisées }\end{array}$ & & $\mathrm{X}$ \\
\hline 4.5 .4 & Dépenses d'éducation par élève, par niveau d'enseignement et source de financement & $\mathrm{x}$ & \\
\hline 4.5 .5 & Pourcentage de l'aide totale à l'éducation alloué aux pays à faible revenu & $\mathrm{x}$ & \\
\hline \multicolumn{4}{|c|}{$\begin{array}{l}\text { Cible 4.6 D'ici à 2030, veiller à ce que tous les jeunes et une proportion considérable d'adultes, hommes et femmes, sachent lire, écrire e } \\
\text { compter }\end{array}$} \\
\hline 4.6.1 & $\begin{array}{l}\text { Pourcentage de la population d'une tranche d'âge donnée atteignant au moins un certain niveau de } \\
\text { maîtrise de compétences fonctionnelles en matière (a) de lecture et d'écriture et (b) de calcul, par sexe }\end{array}$ & $\mathrm{X}$ & $x$ \\
\hline 4.6 .2 & Taux d'alphabétisation des jeunes et des adultes & $\mathrm{x}$ & \\
\hline 4.6.3 & Taux de participation des jeunes et des adultes aux programmes d'alphabétisation & & $x$ \\
\hline \multicolumn{4}{|c|}{$\begin{array}{l}\text { Cible 4.7 D'ici à 2030, faire en sorte que tous les élèves acquièrent les connaissances et compétences nécessaires pour promouvoir le } \\
\text { développement durable, notamment par l'éducation en faveur du développement et de modes de vie durables, des droits de } \\
\text { l'homme, de l'égalité des sexes, de la promotion d'une culture de paix et de non-violence, de la citoyenneté mondiale et de } \\
\text { l'appréciation de la diversité culturelle et de la contribution de la culture au développement durable }\end{array}$} \\
\hline 4.7.1 & $\begin{array}{l}\text { Mesure dans laquelle (i) l'éducation à la citoyenneté mondiale et (ii) l'Éducation en vue du développement } \\
\text { durable, y compris l'égalité entre les sexes et les droits de la personne, sont intégrées à tous les niveaux } \\
\text { dans: (a) les politiques nationales d'éducation, (b) les programmes scolaires, (c) la formation des } \\
\text { enseignants et (d) l'évaluation des élèves }\end{array}$ & $x$ & $\mathrm{x}$ \\
\hline 4.7 .2 & $\begin{array}{l}\text { Pourcentage d'écoles qui dispensent une éducation au VIH et à la sexualité basée sur les compétences } \\
\text { utiles dans la vie }\end{array}$ & & $x$ \\
\hline 4.7 .3 & $\begin{array}{l}\text { Mesure dans laquelle le cadre du Programme mondial d'éducation dans le domaine des droits de l'homme } \\
\text { est mis en œuvre à l'échelle nationale (conformément à la résolution 59/113 de l'Assemblée générale des } \\
\text { Nations Unies) }\end{array}$ & & $x$ \\
\hline
\end{tabular}




\begin{tabular}{|l|l|c|}
\hline 4.7.4 & $\begin{array}{l}\text { Pourcentage d'élèves par tranche d'âge (ou d'un niveau d'études) montrant une compréhension adéquate } \\
\text { des questions relatives à la citoyenneté mondiale et à la durabilité }\end{array}$ & 2017 \\
\hline 4.7 .5 & Pourcentage d'élèves de 15 ans montrant une maîtrise des sciences de l'environnement et de la Terre & $X$ \\
\hline
\end{tabular}

Cible 4.a Faire construire des établissements scolaires qui soient adaptés aux enfants, aux personnes handicapées et aux deux sexes ou adapter les établissements existants à cette fin et fournir un cadre d'apprentissage effectif qui soit sûr, exempt de violence et accessible à tous

\begin{tabular}{|l|l|c|c|}
\hline $4 . a .1$ & $\begin{array}{l}\text { Pourcentage d'écoles disposant : (a) de l'électricité ; (b) de l'Internet à des fins pédagogiques ; } \\
\text { (c) d'ordinateurs à des fins pédagogiques; }(\mathrm{d}) \text { d'infrastructures et de matériel adaptés aux élèves } \\
\text { handicapés; (e) d'un accès élémentaire à l'eau potable ; (f) d'installations sanitaires de base séparées pour } \\
\text { hommes et femmes ; et (g) d'un minimum d'installations pour le lavage des mains (selon les définitions de } \\
\text { l'indicateur WASH) }\end{array}$ & X & X pour (d) \\
\hline $4 . a .2$ & $\begin{array}{l}\text { Pourcentage d'élèves victimes d'intimidation, de châtiment corporel, de harcèlement, de violence, de } \\
\text { discrimination et d'abus sexuels }\end{array}$ & $X$ \\
\hline 4.a.3 & Nombre d'attaques sur les élèves, le personnel et les établissements & $X$ \\
\hline
\end{tabular}

Cible 4.b D'ici à 2030, augmenter considérablement à l'échelle mondiale le nombre de bourses d'études offertes aux pays en développement, en particulier aux pays les moins avancés, aux petits États insulaires en développement et aux pays d'Afrique, pour financer le suivi d'études supérieures, y compris la formation professionnelle, les cursus informatiques, techniques et scientifiques et les études d'ingénieur, dans des pays développés et d'autres pays en développement

\begin{tabular}{|c|c|c|c|}
\hline 4.b.1 & $\begin{array}{l}\text { Volume de l'aide publique au développement consacrée aux bourses d'études, par secteur et type de } \\
\text { formation }\end{array}$ & $\mathrm{X}$ & \\
\hline 4.b.2 & Nombre de bourses d'enseignement supérieur accordées par pays bénéficiaire & & $\mathrm{X}$ \\
\hline \multicolumn{4}{|c|}{$\begin{array}{l}\text { Cible 4.c D'ici à 2030, accroître considérablement le nombre d'enseignants qualifiés, notamment au moyen de la coopération internationale } \\
\text { pour la formation d'enseignants dans les pays en développement, surtout dans les pays les moins avancés et les petits États } \\
\text { insulaires en développement }\end{array}$} \\
\hline 4.c.1 & $\begin{array}{l}\text { Pourcentage d'enseignants dans : (a) le préscolaire ; (b) le cycle primaire ; (c) le premier cycle du } \\
\text { secondaire ; et (d) le second cycle du secondaire qui ont au moins reçu (avant leur entrée en fonction ou } \\
\text { en cours d'activité) les formations minimum organisées pour les enseignants (notamment dans le domaine } \\
\text { pédagogique), requises pour l'enseignement à un niveau pertinent dans un pays donné, par sexe }\end{array}$ & $x$ & \\
\hline 4.c.2 & Ratio élèves/enseignants formés, par niveau d'études & $\mathrm{X}$ & \\
\hline 4.c.3 & $\begin{array}{l}\text { Pourcentage d'enseignants qualifiés selon les normes nationales, par niveau d'études et type } \\
\text { d'établissement }\end{array}$ & $x$ & \\
\hline 4.c. 4 & Ratio élèves/enseignants qualifiés, par niveau d'études & $x$ & \\
\hline 4.c.5 & $\begin{array}{l}\text { Salaire moyen des enseignants par rapport aux autres professions exigeant un niveau comparable de } \\
\text { formation et de qualification }\end{array}$ & & $\mathrm{x}$ \\
\hline 4.c.6 & Taux d'attrition des enseignants par niveau d'études & $x$ & \\
\hline 4.c.7 & $\begin{array}{l}\text { Pourcentage d'enseignants qui ont bénéficié d'une formation continue au cours des } 12 \text { derniers mois, par type } \\
\text { de formation }\end{array}$ & & $\mathrm{x}$ \\
\hline
\end{tabular}

Note : Les encadrés orange correspondent à des indicateurs mondiaux ; les encadrés bleus à des indicateurs thématiques. Pour en savoir plus sur les définitions, ma méthodologie, l'interprétation et les limites de chaque indicateur, veuillez consulter ISU, 2017d.

Source : GDCT, 2017. 


\section{Niveau 1}

Niveau 1 : L'indicateur est conceptuellement clair et a une méthodologie et des normes établies au plan international. En outre, des données sont produites régulièrement par au moins $50 \%$ des pays sur au moins la moitié de leur population dans chaque région où l'indicateur est pertinent.
4.2 .2
4.b. 1

\section{Niveau 2}

L'indicateur est conceptuellement clair, a une méthodologie et des normes établies au plan international, mais les données ne sont pas régulièrement produites par les pays.
$4.1 .1(b)$
(b) et (c)
4.3.1
4.4.1
4.6.1
4.c. 1

\section{Niveau 3}

Aucune méthodologie ou norme n'est à ce jour disponible pour l'indicateur, mais la méthodologie/normes sont en cours d'élaboration ou en phase d'essai (ou le seront prochainement).
4.1.1 (a)
4.2.1
4.7.1

\section{Niveaux variables}

4.a.1 Niveau 1/2/3 en fonction du composant

4.5.1 Niveau 1/2/3 en fonction de l'indicateur sous-jacent

Note : La classification par niveau date du 20 avril 2017, mais inclut la reclassification de 4.c.1 à la sixième réunion du GIAE-0DD. Source : UNDESA, 2016. 
que lors de ces examens. Les membres du GIAEODD déterminent la portée des examens majeurs et élaboreront des propositions pour ajouter, éliminer ou modifier une sélection d'indicateurs. Ils réaliseront une série de consultations ouvertes sur les changements proposés auprès des pays observateurs, des organisations régionales et internationales, de la société civile et des autres parties prenantes. Des ajouts peuvent être effectués lorsque les indicateurs mondiaux existants ne couvrent pas de façon appropriée l'intention réelle d'une cible donnée ou lorsque des indicateurs mondiaux existants ne sont toujours pas disponibles au moment de l'examen majeur. Des suppressions d'indicateurs peuvent être envisagées lorsque des méthodologies appropriées ne peuvent être mises au point pour un indicateur de Niveau 3 où lorsque des indicateurs existants ne sont pas suffisamment puissants pour mesurer les progrès réalisés. Des modifications peuvent s'avérer nécessaires à des fins de clarification, de simplification ou lorsqu'une définition plus large ou une discrimination est nécessaire.

Il est prévu que la majorité des indicateurs de l'ODD 4 du cadre mondial actuel seront conservés. En 2016, le GIAE-ODD a manifesté un intérêt à envisager des indicateurs mondiaux supplémentaires pour certaines cibles. Parmi ceux-ci, il y avait les taux d'enfants non scolarisés, les taux d'achèvement et le nombre d'années d'éducation gratuite - qui sont tous des indicateurs thématiques - pour la cible 4.1. Le GIAE-ODD voudrait également élargir l'indicateur 4.b.1 relatif aux dépenses consacrées aux bourses pour les études à l'étranger de façon à ce qu'il inclue les programmes de bourses d'études privés. Compte tenu de la grande diversité des fournisseurs de bourses, ces données ne sont pas, à ce jour, collectées systématiquement et de façon exhaustive et ne peuvent être agrégées de manière fiable.

\subsubsection{Le cadre des indicateurs thématiques}

L'élaboration du cadre des indicateurs thématiques de l'ODD 4 est le fruit de travaux présidés par l'ISU qui ont commencé avec le processus d'élaboration des indicateurs de l'éducation pour le Cadre d'action Éducation 2030. En mars 2014, I'UNESCO a créé le Groupe consultatif technique sur les indicateurs de l'éducation post-2015 (GCT) qui avait notamment pour mission de sélectionner un ensemble d'indicateurs afin de surveiller l'ODD 4, qui a finalement été inclus en tant que projet dans le Cadre d'action Éducation 2030. La sélection des 43 indicateurs effectuée par le GCT - 11 indicateurs mondiaux et 32 indicateurs thématiques - reposait sur cinq critères, à savoir la pertinence, l'alignement avec les concepts de la cible, la faisabilité de la collecte régulière des données dans les pays, la facilité de la communication à un public mondial et l'interprétabilité (ISU, 2017e; UNESCO, 2015).

Le Cadre d'action Éducation 2030 exigeait que I'ISU travaille avec les partenaires et les conduise à collecter les données, à élaborer des indicateurs et à renforcer les systèmes de données nationaux. En 2016, l'ISU a réuni le Groupe de coopération technique (GdCT) sur les indicateurs du Cadre d'action Éducation 2030 - ODD 4 afin de conduire l'élaboration méthodologique et la mise en œuvre du cadre des indicateurs thématiques, conçu pour surveiller complètement les indicateurs mondiaux liés à l'éducation. Le GdTC réunit des experts représentatifs au plan régional de 28 États membres (la même représentation régionale que celle du GIAE-ODD et le Royaume-Uni, un ancien membre), l'équipe du RMSE, l'OCDE, I'UNESCO, I'UNICEF, la Banque mondiale et des organisations de la société civile, ainsi que des observateurs de commissions et d'institutions régionales et de pays du Comité directeur de l'agenda Éducation 2030. L'ISU accueille le Secrétariat et copréside le GdCT avec la Division de I'UNESCO pour l'appui de l'agendaÉducation 2030.

En octobre 2016, le GdCT a approuvé un ensemble de 29 indicateurs (11 mondiaux et 18 thématiques) prêts pour l'établissement de rapports en 2017, sélectionnés parmi la liste originale des 43 indicateurs proposés (voir Tableau 1). Le Comité directeur sur l'ODD 4-Éducation 2030 - un mécanisme de coordination accueilli par l'UNESCO pour soutenir les États membres et les partenaires - a entériné les 29 indicateurs lors de sa réunion de décembre 2016, apportant de ce fait un soutien politique important pour leur adoption par les pays. Bien que le Comité directeur ait un rôle permanent de coordination, il ne revient pas sur les discussions techniques ayant trait à la sélection des indicateurs.

En 2016, I'ISU a également créé l'Alliance mondiale pour le suivi de l'apprentissage (GAML) afin de donner des conseils sur le développement méthodologique des indicateurs des résultats d'apprentissage liés 
à l'ODD 4 (mondiaux et thématiques). La GAML est composée d'un vaste éventail d'experts et de décideurs impliqués dans des initiatives nationales et transnationales d'évaluation des apprentissages, ainsi que de donateurs et d'organisations de la société civile qui défendent l'éducation. La GAML fonctionne par le biais de groupes de travail focalisés sur chaque cible liée à des résultats d'apprentissage $(4.1,4.2 .4 .4 .4 .6$ et 4.7$)$ et d'un groupe de travail transversal qui élabore un Cadre d'assurance de la qualité des données (DQAF) pour les évaluations des apprentissages. Les experts de la GAML dirigent également l'élaboration de normes et de bonnes pratiques d'évaluation des apprentissages.

Lorsque le GdCT a approuvé les 29 indicateurs pour les rapports de 2017, il a également identifié 22 indicateurs qui devaient faire l'objet d'un développement méthodologique approfondi. Quatorze d'entre eux sont les indicateurs thématiques restants parmi les 43 indicateurs initiaux, et huit autres sont des indicateurs mondiaux inclus dans la liste des 29 indicateurs à utiliser pour la production de rapports en 2017 (voir Tableau 1). Le GdCT a créé le Groupe de travail sur l'élaboration d'indicateurs (GT-El) - composé de huit des dix participants au GdCTd - qui a pour mission de finaliser les méthodologies de 15 des 22 indicateurs ${ }^{8}$. La GAML élaborera des méthodologies pour les sept indicateurs restants, qui concernent les résultats d'apprentissage. Depuis le début de 2017, le GT-EI et les groupes de travail de la GAML examinent les méthodologies et sources de données existantes pour leurs ensembles d'indicateurs respectifs et consultent des experts externes en tant que de besoin. Ils ont pour objectif de faire des recommandations au GdCT sur les méthodologies les plus appropriées pour les 22 indicateurs, et d'achever ainsi les travaux d'élaboration de l'ensemble des 43 indicateurs d'ici fin 2018. Lorsque le GdCT aura approuvé les méthodologies, les 22 indicateurs restants seront prêts pour leur future utilisation dans le cadre de la production des rapports. Par ailleurs, I'ISU soumettra des propositions en vue de l'examen annuel du GIAE-ODD afin que soit modifiée la classification par niveaux des indicateurs mondiaux pertinents. Les travaux du GdCt et de la GAML consisteront également à inclure les conseils de l'ISU et de ses organismes partenaires lors des examens majeurs du
GIAE-ODD, en 2019 et 2024.

\subsection{Le défi de la production des données requises pour les indicateurs}

La capacité statistique de la plupart des pays est mise à l'épreuve par l'ampleur, l'importance et l'ambition des objectifs mondiaux relatifs à l'éducation. L'importance accordée à l'équité et à la qualité de l'éducation dans le Programme de développement durable à l'horizon 2030 et le caractère plus exhaustif des ODD par rapport aux OMD exigent davantage de données qu'auparavant et un éventail de sources encore plus large : des données administratives, des données financières, des recensements, des enquêtes auprès des ménages, ainsi que les évaluations des apprentissages effectuées aux plans national et international. Les exigences en matière de données impliquent également une coopération accrue entre les différents ministères nationaux, institutions et autres dépositaires de données afin de couvrir l'ODD dans toute son ampleur, des programmes de soins et d'éducation en faveur de la petite enfance à l'enseignement supérieur et à l'apprentissage tout au long de la vie. Les contributions apportées par d'autres secteurs, tels que la santé, les affaires féminines et le travail, sont nécessaires pour produire les données des indicateurs liés à l'éducation des autres ODD (UNESCO, 2016).

Une évaluation des capacités statistiques des pays en matière de collecte des données de l'ODD 4 a récemment mis au jour l'ampleur de la tâche que représente la production d'indicateurs de la qualité. En 2016, I'ISU a procédé à des évaluations de la disponibilité des données au niveau national pour surveiller l'ODD 4. Des employés responsables des statistiques sur l'éducation de 121 pays - dans les États arabes, en Asie et dans le Pacifique, en Amérique latine et aux Caraïbes et en Afrique subsaharienne - ont déterminé s'ils produisaient déjà régulièrement les données nécessaires pour les 43 indicateurs mondiaux et thématiques. Moins de la moitié (47\%) avaient des données suffisantes pour les 11 indicateurs mondiaux, mais près des deux tiers (63\%) ont indiqué avoir les données nécessaires pour calculer les 32 indicateurs

\footnotetext{
${ }^{8}$ Chacun des Groupes de travail du GdCT comprend entre six et dix membres autodésignés, comprenant généralement au moins trois pays membres du GdCT, deux représentants de la société civile ou d'organisations partenaires, et un membre du personnel de l'ISU agissant en tant que Secrétaire. Des observateurs du GdCT peuvent être conviés à se joindre aux groupes de travail.
} 
thématiques restants (sans compter les indicateurs mondiaux). Lorsque les données sont disponibles, la qualité et l'étendue de la collecte des données ne correspondent pas toujours aux attentes de l'ODD 4. Ventiler les données par mesures de la richesse et par degrés d'incapacité, par exemple, n'a été respectivement possible que dans $14 \%$ et $19 \%$ des pays. Certains concepts de l'ODD 4 sont plus susceptibles d'être déjà collectés, comme les données sur la participation et l'achèvement, qui sont disponibles dans $85 \%$ des pays. Les données sur les connaissances, les compétences, l'aptitude à apprendre et la maturité scolaire, cependant, ne sont disponibles que dans $43 \%$ des pays qui ont répondu (ISU, 2016).

La couverture des pays dans la base de données de l'ISU est inférieure à ce que suggérait l'évaluation de 2016. La Figure 2 montre la disponibilité des indicateurs mondiaux et thématiques dans l'ensemble des pays qui ont fourni des données à la base de données de
I'ISU en 2017 : 10 des 43 indicateurs n'étaient disponibles dans aucun des pays, et 8 indicateurs mondiaux et 11 indicateurs thématiques n'ont été documentés que dans $50 \%$ ou moins des pays. Seul un indicateur mondial et six indicateurs thématiques sont disponibles dans plus de 75 $\%$ des pays. Les pays ont du mal à documenter et, dans de nombreux cas, à collecter les données nécessaires pour calculer les indicateurs indispensables au suivi et à l'examen de l'ODD 4 (ISU, 2017c).

Le Programme de développement durable à I'horizon 2030 et le Cadre d'action Éducation 2030 font expliCITÉment appel aux organisations internationales et autres parties prenantes disposant d'une expertise technique pour qu'elles contribuent au renforcement des capacités et aux efforts de collecte des données nécessaires pour effectuer le suivi des ODD. Le GdCT a montré la voie en aidant les pays à accroître leurs capacités techniques à collecter et à présenter des données

\section{Figure 2. Disponibilité des indicateurs mondiaux et thématiques de I'ODD 4 dans la base de données de I'ISU, juin 2017}

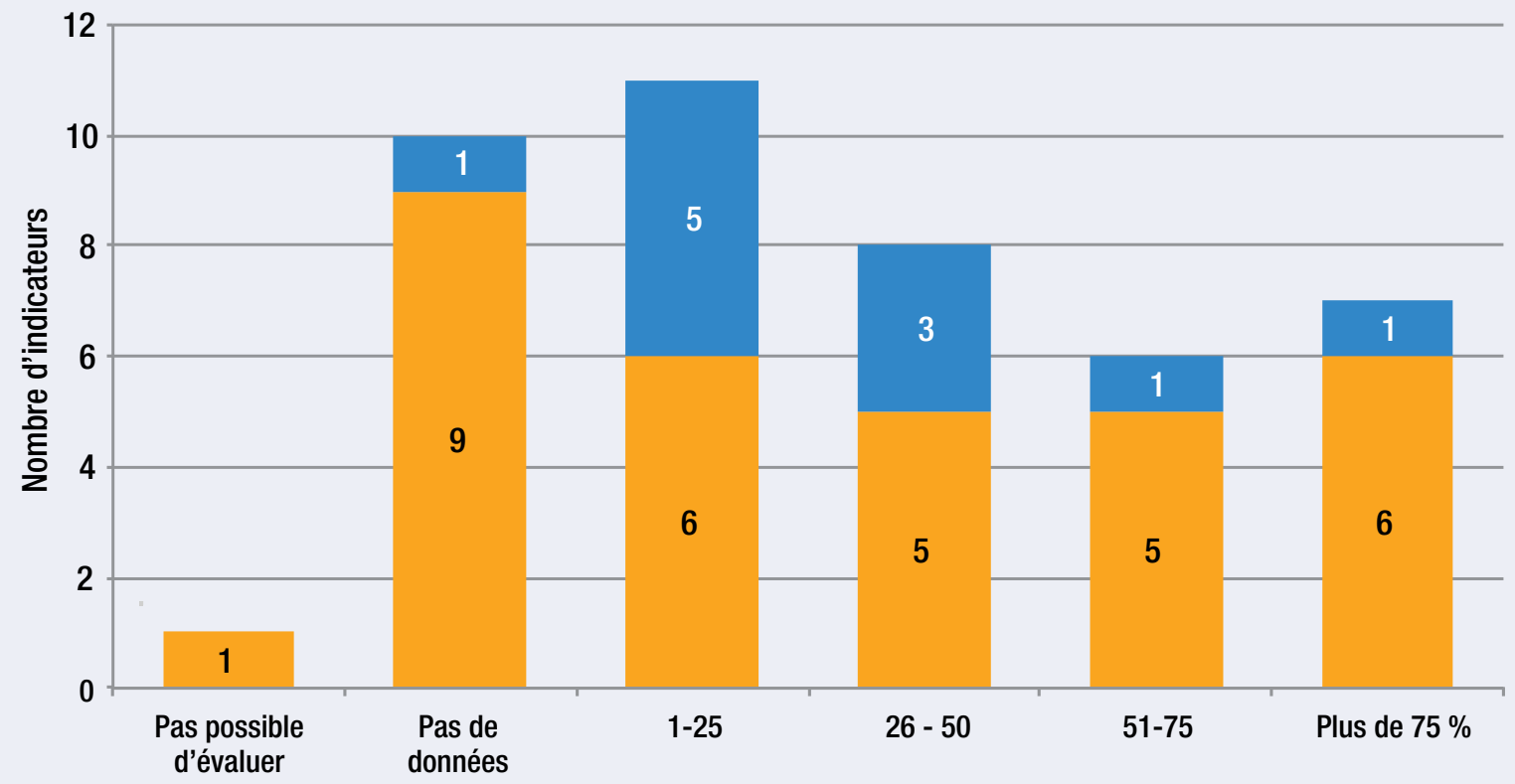

Couverture des pays ( $\%$ des pays)

Indicateurs globaux $\quad$ Indicateurs thématiques 
pour les indicateurs de l'ODD 4. Début 2017, il a créé le Groupe de travail sur le renforcement des capacités statistiques (GT-RCS) et le Groupe de travail sur la communication, la validation et la diffusion des données (GT-CVD) qui complètent le GT-El susmentionné. Les groupes de travail transmettent le fruit de leurs travaux au GdCT, qui prend les décisions sur la base de leurs recommandations.

Le GT-RCS est en train d'élaborer un cadre d'instruments et de principes directeurs du renforcement des capacités pour aider les pays à évaluer leurs besoins spécifiques en matière de développement des capacités et à identifier des sources de soutien. Le cadre recueillera des instruments et principes directeurs existants auprès de différentes sources. Le groupe de travail identifiera également les lacunes du cadre et fera des recommandations en vue de l'élaboration d'instruments et de principes directeurs supplémentaires.

Le GT-CVD cartographie le flux de données circulant des pays vers les organisations internationales puis vers la DSNU et incluses dans la base de données mondiale des indicateurs des ODD. Le groupe préparera le calendrier de chacun des exercices de collecte de données et désignera les organisations responsables. II décrira les processus de l'assurance qualité ainsi que les cycles de rétroaction permettant aux pays de valider les résultats finals. Le groupe fera des recommandations sur un protocole entre les pays et les organisations assurant un flux de données efficace et transparent et un consensus sur les résultats à publier.

Le Programme de développement durable à l'horizon 2039 et le Cadre d'action Éducation 2030 appellent également à la mobilisation du financement public international - et surveillent, notamment, l'aide au développement - afin de contribuer à la réalisation des ODD et de compléter les ressources nationales, en particulier dans les pays les plus pauvres. L'ISU a estimé que satisfaire aux besoins de données de l'agenda Éducation 2030 coûterait environ 2,2 milliards de dollars des États-Unis pendant une dizaine d'années ; $43 \%$ de ce montant implique l'évaluation régulière, sur la base d'échantillons, des résultats d'apprentissage dans les premières et dernières années du primaire (ISU, 2017i). Le secteur privé est encouragé à contribuer aux coffres de l'éducation, tout en préservant le respect de la responsabilité, de la transparence et de l'équité, et en partenariat avec le secteur public. L'ISU a spécifiquement recommandé que le secteur privé mobilise des contributions en nature afin de soutenir l'amélioration de la collecte et de la production des données (ISU, 2017i).

Renforcer la capacité d'un pays à collecter et à communiquer les données est la première étape nécessaire à la production d'indicateurs comparables au plan international. L'autre étape indispensable est l'évaluation de la qualité des données en cours de production par rapport aux normes de qualité internationales. C'est le sujet de la section 2. Par conséquent, jeter les bases statistiques afin de surveiller de façon appropriée les progrès réalisés sur la voie de l'objectif et des cibles liés à l'éducation est une activité qui doit être focalisée sur la production de données de qualité.

Le terme " qualité » est interprété au sens large, et englobe la mesure dans laquelle les processus statistiques et leurs résultats satisfont les attentes des utilisateurs et parties prenantes (voir Encadré 2). Au cours des 20 dernières années, les instituts de la statistique sont parvenus au consensus selon lequel le concept de qualité des informations statistiques est multidimensionnel, et qu'il n'existe pas de mesure unique de la qualité des données. Des efforts particuliers devront être déployés pour s'assurer que la qualité soit un objectif pendant toutes les phases de la production (développement, collecte, traitement, validation et diffusion).

Favoriser ces efforts d'une façon systématique, pour tous les mécanismes nationaux et internationaux de production des données et pour un large éventail de sources de données, est l'objectif de plusieurs des efforts déployés par I'ISU. La Section 2 discute des initiatives menées récemment pour promouvoir un niveau de qualité normalisé pour l'ensemble des efforts de collecte de données relatives à l'ODD 4. La Section 3 examine des stratégies de développement des capacités spécifiques visant à soutenir les systèmes nationaux de statistiques sur l'éducation. 
Les concepts présentés ci-après sont souvent utilisés pour évaluer collectivement la qualité des processus de collecte et de diffusion des données. II n'est pas nécessaire que tous les concepts satisfassent aux normes les plus strictes pour que la qualité des données soit jugée acceptable, mais il est essentiel d'utiliser une sélection de ces concepts comme repère pour valider la qualité des données produites.

Pertinence. La pertinence d'une production statistique est la mesure dans laquelle les données atteignent l'objectif pour lequel elles sont recherchées par les utilisateurs.

Exactitude. L'exactitude d'une production statistique fait référence au degré de correspondance entre les données et les quantités ou caractéristiques qu'elles estiment ou mesurent. L'exactitude fait référence à la proximité entre les valeurs statistiques et les valeurs réelles (inconnues).

Fiabilité. La fiabilité fait référence à la similitude entre les valeurs initialement publiées d'une production statistique et les valeurs qui sont ultérieurement publiées pour la même période de référence.

Cohérence. La cohérence fait référence à la mesure dans laquelle une production statistique est logiquement connectée et mutuellement compatible avec d'autres productions statistiques. Selon le principe de cohérence, un même terme ne peut être utilisé pour des concepts différents sans définition préalable.

Actualité. L'actualité d'une production statistique est la période de temps entre sa disponibilité et l'événement ou phénomène qu'elle décrit. L'actualité est évaluée en termes d'échelle de temps qui dépend de la période pendant laquelle les données ont une valeur, c'est-à-dire pendant laquelle elles sont assez pertinentes pour appuyer des actions.

Ponctualité. La ponctualité d'une production statistique implique l'existence et le respect d'un calendrier de diffusion de la production.
Une production est ponctuelle si elle est diffusée conformément au calendrier.

Accessibilité. L'accessibilité d'une production statistique fait référence à la facilité avec laquelle les données peuvent être découvertes, localisées et extraites des archives de données. Elle comprend l'adéquation des formats dans lesquels les données sont disponibles, les moyens de diffusion, la disponibilité des métadonnées et les services d'appui aux utilisateurs, et, dans le cas où elles sont vendues, l'abordabilité des données proposées aux utilisateurs.

Interprétabilité. L'interprétabilité ou la clarté d'une production statistique fait référence à la facilité avec laquelle les utilisateurs peuvent comprendre les données et les utiliser de façon appropriée. Le degré d'interprétabilité dépend en grande partie des métadonnées qui accompagnent les données, notamment les définitions de concepts, les populations cibles, les indicateurs et autres éléments terminologiques décrivant la production et ses limites.

Objectivité. Les méthodes et productions statistiques sont déterminées par des considérations statistiques et non par les pressions exercées par les fournisseurs, utilisateurs et autres parties prenantes.

Impartialité. Les commentaires et les communiqués de presse sont objectifs et non partisans.

Transparence. Les utilisateurs sont informés des sources et des méthodes, ainsi que des changements qui peuvent leur être apportés et qui peuvent affecter les productions. Les limites des productions et des processus grâce auxquels elles sont réalisées sont indiquées.

Crédibilité : Il s'agit de la confiance des utilisateurs envers leurs produits. Elle repose essentiellement sur l'image qu'ils se sont forgée du producteur et de ses productions statistiques, ainsi que de leur confiance envers l'objectivité et l'impartialité des méthodes utilisées. 


\section{Produire des statistiques de qualité pour surveiller les progrès réalisés sur la voie de l'ODD 4}

En tant que dépositaire de la majorité des statistiques internationales sur l'éducation utilisées pour le Cadre d'action Éducation 2030 - ODD 4, I'ISU s'est donné comme vision de produire des données de qualité pour évaluer les progrès sur la voie des objectifs internationaux liés à l'éducation. En réfléchissant à l'ambitieux agenda Éducation, l'ISU et la communauté internationale des statistiques ont identifié des lacunes en matière de données et d'autres défis méthodologiques auxquels est confrontée l'élaboration d'indicateurs mondiaux et thématiques de l'éducation. Le principal défi, cependant, consiste à établir et à renforcer une production systématique et durable de données sur l'éducation de la qualité requise. Ceci jetterait les bases de nouvelles approches méthodologiques et de nouveaux efforts en matière de collecte de données.

En 2017, I'ISU a lancé un appel urgent en faveur d'une révolution des données sur l'éducation afin de lever les obstacles qui entravent la production de données de qualité destinées à surveiller l'ODD 4. S'appuyant sur ses décennies d'expérience dans l'amélioration des systèmes de statistiques nationaux sur l'éducation et de collecte des données internationales sur l'éducation, I'ISU reconnaît que la nature ambitieuse de l'ODD 4 exige de nouvelles approches méthodologiques pour évaluer et améliorer la production de données de qualité sur l'éducation, tant au niveau national qu'international. L'ISU comprend également qu'il est nécessaire de considérer les objectifs stratégiques de la production de données de qualité d'un point de vue à la fois national et international. Au niveau national, les exigences en matière de qualité sont le reflet de l'utilisation des données pour surveiller les pratiques et les politiques nationales et pour apporter aux défis politiques des réponses fondées sur les faits. Au niveau international, les besoins en données de qualité sont en lien avec la fiabilité, l'actualité et la comparabilité des données transnationales sur l'éducation.
Établir et renforcer systématiquement la qualité des données, aux niveaux national et international, pose par conséquent des problèmes significatifs et distincts. Cependant, la qualité des données reflète la qualité du processus qui a produit les données, et la solidité du cadre de suivi mondial de l'ODD 4 repose que la qualité des données produites au niveau national. Cette section commence par décrire la vision de l'ISU en matière de données de qualité et la nécessité d'une révolution des données sur l'éducation. Elle décrit ensuite les mesures prises par l'ISU pour améliorer les normes de qualité des données utilisées pour surveiller le Cadre d'action Éducation 2030 - ODD 4 aux niveaux national et international.

\subsection{Une révolution des données sur l'éducation}

L'ISU a appelé à une révolution des données sur l'éducation afin d'établir un système efficace de suivi des progrès sur la voie des ODD qui satisfasse aux normes de production de données de qualité (voir Section 1.3). Les principaux objectifs de la révolution des données sur l'éducation répondent à la nécessité de combler les multiples « lacunes en termes de normes et de standards, de disponibilité des données, de méthodes pour mesurer les indicateurs fondamentaux, et de coordination entre les différentes parties prenantes qui affectent toute la chaîne de la production et de l'utilisation des données » (ISU, 2017i, p. 48).

Le modèle de révolution des données de l'ISU exige de renforcer les systèmes de statistiques nationaux qui constituent les fondements du système de suivi et de créer des mécanismes de soutien internationaux en vue de la production efficace d'indicateurs comparables au plan international ${ }^{9}$. La communauté internationale, qui comprend les acteurs publics et privés, apporte un soutien crucial à la réalisation du programme mondial de suivi de l'ODD 4. Chacune des

\footnotetext{
${ }^{9}$ Pour en savoir plus sur les défis sous-jacents des processus de données sur l'éducation, voir ISU, 2017i.
} 
composantes clés de la révolution des données comprend trois vastes domaines cibles aux niveaux national et international. Les trois piliers, autour desquels s'articulent les Principes fondamentaux des statistiques officielles, comprennent : des environnements favorables, la production des données et la diffusion des données (voir Figure 3).
- Environnements favorables : Des investissements dans les ressources techniques et financières afin de relever les défis auxquels sont confrontés les instituts de statistiques nationaux; des investissements dans les technologies et des effectifs formés ; la gouvernance des institutions responsables de l'éducation et des instituts de statistiques nationaux.

\section{Figure 3. Composantes de la révolution des données sur l'éducation}

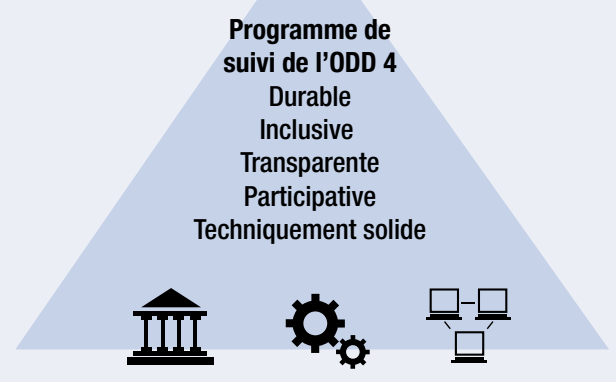

\section{Soutien international en tant que facteur favorable}

\begin{abstract}
Environnement favorable
Rôles et responsabilités définis précisément

Approche participative de l'élaboration des méthodologies

Méthodologies sensibles à l'inclusion de toutes les populations
\end{abstract}

Production de données solide Transparence

Solidité

Validation avec les pays

Inclusion de toutes les sources de données possibles

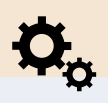

Utilisation et diffusion des données Réduction des coûts interinstitutions des échanges de données

Transfert aux pays des connaissances et de la propriété

Processus d'apprentissage entre pairs

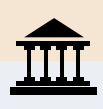

Systèmes statistiques des pays comme fondement

\section{Environnement favorable}

Stratégies nationales pour les statistiques de l'éducation

Aligner : - les données avec les priorités nationales

- l'engagement politique et les ressources avec les besoins de données

Collaborations interministérielles et intraministérielles et implication des parties prenantes

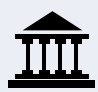

Solidité de la production des données

Système national de statistiques sur l'éducation : clé de voûte des instruments de suivi nationaux et mondiaux

Intégration des sources de données de façon à couvrir toutes les cibles de l'ODD4 et à améliorer le contrôle de la qualité

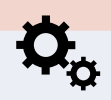

Utilisation et diffusion des données

Amélioration de la maîtrise des données et de l'utilisation des statistiques de l'éducation dans l'élaboration des politiques

Facilité d'utilisation pour les différentes parties prenantes, y compris les enseignants et les familles Diffusion des métadonnées

Source : Adaptation d'un document de I'Institut de statistique de I'UNESCO, $2017 i$. 
- Production des données : Amélioration de la collecte des données conformément aux normes et standards internationaux ; priorisation, par les gouvernements, de la collecte régulière des données; harmonisation des normes de collecte des données dans certains domaines (par exemple, les résultats d'apprentissage) ; élargissement à d'autres sources de données fiables.

\section{- Utilisation et diffusion des données :}

Renforcement des mécanismes d'utilisation et de diffusion des données ; utilisation des technologies pour élargir l'impact des données et des informations.

La discussion du rôle et des activités actuels de l'ISU dans l'amélioration de la qualité des données sur l'éducation aux niveaux national et international est le sujet des parties suivantes de la Section 2. Pour servir de base à ces travaux, I'ISU a élaboré un Code de pratiques (CdP), qui est mis en œuvre par l'intermédiaire du Cadre d'assurance de la qualité des données de l'éducation (Ed-DQAF). Le CdP est un ensemble de principes statistiques - mesurés par des indicateurs - qui définissent les éléments nécessaires pour produire des données de qualité dans un environnement institutionnel, ainsi que les processus statistiques et les résultats statistiques liés à l'éducation. Appliqué au niveau national, l'Ed-DQAF intègre le CdP dans une série d'activités et de processus à l'aune desquels un pays peut mesurer son système de production de données. Bien que le CdP ait été produit par l'ISU en 2017 en tant que document autonome, dans la pratique il était déjà intégré dans la méthodologie de l'Ed-DQAF mise en œuvre par l'ISU depuis 2005.

Il est également important de prendre acte du rôle des organisations internationales, des partenaires financiers mondiaux, des défenseurs de l'éducation et d'autres groupes qui soutiennent activement les efforts nationaux et régionaux visant à produire des statistiques de qualité. Dans le cadre de cette stratégie de travail, des codes de pratiques similaires, visant à améliorer la production de statistiques de qualité, ont été adoptés par des organisations internationales, des partenaires financiers mondiaux et d'autres groupes régionaux.
L'un des meilleurs exemples est le Code de bonnes pratiques de la statistique européenne, adopté pour la première fois par le Comité du système statistique européen en 2005. L'édition révisée de 2011 comprend un ensemble de 15 principes clés qui guident la production et la diffusion des statistiques européennes officielles et offrent un environnement institutionnel standard dans le cadre duquel fonctionnent les autorités nationales et européennes de la statistique (Eurostat et le Système statistique européen). Publié en 2011, le Cadre d'assurance qualité (QAF) du Système statistique européen fournit des informations sur la façon de mettre en œuvre le Code de bonnes pratiques de la statistique européenne. II contient des recommandations d'activités, des méthodes et des outils pour améliorer la mise en œuvre des indicateurs en examinant l'environnement institutionnel, les processus statistiques et les résultats statistiques (SSE, 2015; Eurostat, 2011).

Le CdP européen et le QAF ont servi de modèles à des organisations régionales lorsqu'elles ont élaboré leurs propres outils, notamment en Amérique latine et aux Caraïbes (voir Encadré 3). En élaborant des cadres d'évaluation régionaux, les réseaux et organisations régionaux définissent une norme régionale en vue de l'amélioration des systèmes statistiques et de leurs environnements favorables. L'amélioration de la production des données devient ainsi un objectif collectif au sein d'un groupe de pairs.

Au cours de ces dernières années, la communauté statistique des Nations Unies a également élaboré et adopté deux produits d'assurance de la qualité : le Cadre national d'assurance de la qualité (NQAF) à l'intention des pays et le Cadre d'assurance de la qualité des statistiques (UN SQAF) à l'intention des institutions de l'ONU qui produisent des statistiques (voir Encadré 4).

Au niveau national, plusieurs expériences ont été menées pour mettre en œuvre des cadres d'assurance de la qualité dans des pays développés tels que le Canada, la France, les Pays-Bas et le Royaume-Uni. Cette approche a également été adoptée par certains pays en développement : le cas de l'institut national de la statistique de la Colombie, Departamento Administrativo Nacional de Estadística (DANE), est un bon exemple de production d'un cadre national d'assurance de la qualité des données qui a permis d'améliorer les systèmes statistiques nationaux (voir Encadré 5) ${ }^{10}$.

\footnotetext{
${ }^{10}$ Pour consulter la liste complète des références en matière d'assurance qualité, voir site Web de la DSNU : https://unstats.un.org/unsd/dnss/ QualityNQAF/nqaf.aspx.
} 
En 2007, plusieurs pays de la région Amérique latine et Caraïbes ont proposé à la Conférence statistique des Amériques de la Commission économique pour l'Amérique latine et les Caraïbes (CSA-CEPALC) de prendre note du Code de bonnes pratiques de la statistique européenne et de discuter de la possibilité de l'adapter à la réalité de chaque pays. La CSA-CEPALC a demandé qu'Eurostat et la CEPALC préparent un programme d'action pour mener à bien cette mission.

En novembre 2011, le code de bonnes pratiques des statistiques de l'Amérique latine et des Caraïbes a été adopté par la CSA-CEPALC. Le code est un instrument technique et réglementaire, structuré par des principes et des bonnes pratiques, qui a pour objet de contribuer à l'amélioration de la production des statistiques nationales dans la région. Ses principes sont des règles et des idées fondamentales qui régissent la réflexion et la pratique de l'activité statistique. Les bonnes pratiques statistiques sont considérées comme des actions reproductibles qui reposent sur une expérience reconnue et les meilleurs résultats, et qui contribuent à l'amélioration de l'activité statistique nationale. Le code comprend 17 principes et 84 critères à respecter, groupés en trois sections : environnement institutionnel et coordination, processus statistiques et résultats statistiques.

En tant que document technique, le code contient des règles pratiques permettant d'assurer l'indépendance des instituts nationaux de la statistique et la coordination de la production statistique au niveau national. II sert non seulement de guide pour améliorer la qualité des statistiques dans la région, mais cherche également à coordonner l'activité statistique, à améliorer la qualité des statistiques officielles et à donner confiance aux utilisateurs en encourageant l'application des meilleures méthodes et pratiques internationales dans la production et la diffusion des statistiques.

Sources : CEPALC, 2011a, $2011 b$.

Encadré 4. Le Cadre national d'assurance de la qualité des Nations Unies (NQAF) et le Cadre d'assurance de la qualité des statistiques des Nations Unies (UN SQAF)

En 2012, la DSNU a produit un Cadre national d'assurance de la qualité (NQAF) générique, assorti d'un modèle et de principes directeurs, pour aider les pays à élaborer leurs propres cadres d'assurance de la qualité des statistiques. Le NQAF de l'ONU a été entériné par la CSNU et est progressivement mis en œuvre par les pays. Le modèle est une structure générique dans le cadre de laquelle les pays peuvent formuler et opérationnaliser leurs propres cadres d'assurance de la qualité nationaux ou renforcer les cadres existants. Les principes directeurs soutiennent le modèle en fournissant les listes d'outils et de références spécifiques à la Section 3 du modèle (principes directeurs de l'assurance de la qualité) et à la Section 4 (évaluation de la qualité). Les principes directeurs comprennent une feuille de route détaillée indiquant les liens avec plusieurs cadres d'assurance de la qualité existants ainsi que des liens vers le glossaire en ligne du NQAF.
En 2016, le Comité des statisticiens en chef du système des Nations Unies (CCS UNS) a élaboré le Cadre d'assurance de la qualité des statistiques (UN SQAF). S'appuyant sur des ensembles existants de principes relatifs aux activités statistiques à l'intention des organisations internationales - tels que les Principes régissant les activités statistiques internationales (2014) - I'UN SQAF a pour objectif d'élaborer une interprétation commune des aspects de la qualité et de l'assurance qualité des statistiques aux institutions de l'ONU, qui peut être adapté aux circonstances spécifiques d'une institution particulière. L'UN-SQAF sert également de point d'appui lors de l'examen et de la mise à jour des SQAF par les institutions de I'ONU, notamment des principes directeurs de la coordination et de la promotion de données de meilleure qualité dans l'ensemble du système statistique des Nations Unies. 
L'un des principaux instruments fondateurs du renforcement du système statistique national de la Colombie est son cadre d'assurance de la qualité (Aseguramiento de la calidad de la información estadística), qui est en place depuis 2006.

Pendant les années 1990, la Colombie a cherché à améliorer la qualité des statistiques nationales par le biais de plusieurs initiatives précoces, notamment la création d'un département dédié au sein de l'institut de statistique national Departamento Administrativo Nacional de Estadística (DANE) - afin d'élaborer et d'établir des méthodologies et des processus pour évaluer la qualité des données. Le DANE a bénéficié d'informations et du soutien d'organisations internationales (par exemple CEPALC, FAO) et de chercheurs de différents pays (par exemple le Canada, le Chili, la France, le Mexique) qui ont expliqué les fondements de la production de données de qualité et souligné la nécessité d'évaluer les données à l'aide d'un cadre national d'assurance de la qualité.

En 2006, le cadre national d'assurance de la qualité a été créé par décret gouvernemental au sein du DANE. Les opérations de statistique nationale sont régulièrement évaluées par un processus d'audit et de certification inclus dans le cadre national d'assurance de la qualité qui comprend quatre étapes : la sensibilisation, la collecte des données, l'évaluation et la certification. Entre 2007 et 2016, 255 évaluations de la qualité ont été mises en œuvre et $81 \%$ des opérations statistiques ont été certifiées. Ce processus de certification garantit que les statistiques émanant de différentes parties du système statistique national et infranational sont produites conformément à des normes méthodologiques similaires.

Un agenda de recherche ambitieux examine le cadre national tous les trois ans afin d'améliorer la qualité des statistiques colombiennes. Très récemment, le processus de certification a été révisé afin de l'aligner sur les Principes fondamentaux des statistiques officielles adoptés par l'Assemblée générale de l'ONU en 2014. Le résultat est un cadre institutionnel favorisant la crédibilité, la fiabilité et la transparence lors de la production des statistiques.

Sources : DANE, 2017; OCDE, 2015.

\subsection{Produire des données sur l'éducation au niveau national conformément aux normes de qualité}

\subsubsection{Définir et mettre en œuvre les normes}

Depuis sa création, l'ISU est impliqué dans un large éventail d'activités visant à améliorer la qualité des données sur l'éducation au niveau national. En 2005, I'ISU et la Banque mondiale ont élaboré l'Ed-DQAF, qui est une matrice de notation permettant d'évaluer régulièrement la qualité des données produites par les pays et d'émettre des recommandations en vue de leur amélioration. La référence modèle pour ce document était le cadre d'évaluation générique de la qualité des données produit par le Fonds monétaire international (FMI) autour de six dimensions de la qualité des données : les préalables de la qualité, les assurances de l'intégrité, la solidité méthodologique, l'exactitude et la fiabilité, le fonctionnement et l'accessibilité (FMI, 2012). La matrice est organisée sous forme d'une structure en cascade qui commence par les dimensions générales pour passer ensuite à des détails plus concrets et spécifiques :

- niveau à un chiffre : six dimensions de la qualité s'appuyant sur les cinq proposées par le cadre du FMl et la dimension « 0 » des « préalables de la qualité »; 
- niveau à deux chiffres : sous-dimensions de la qualité ;

- niveau à trois chiffres : indicateurs ;

- niveau à quatre chiffres : bonnes pratiques pour les statistiques de l'éducation.

Les pratiques décrivent les caractéristiques ou exigences - en matière de qualité - qui peuvent être prises en considération lors de l'évaluation des indicateurs. La partie essentielle de l'évaluation consiste à noter chacune de ces pratiques, sur une échelle ordinale allant de 1 (pratique non observée) à 4 (pratique observée). L'évaluation des processus, suivie par celle de l'entité responsable de la source de données, est effectuée par des évaluateurs externes en collaboration avec l'équipe nationale.

La production des indicateurs de l'ODD 4 pose le problème de produire des données nationales sur l'éducation de qualité à une échelle mondiale et qui requièrent, au minimum, un processus systématique. La révolution des données montre la voie en mobilisant différents systèmes produisant des données de qualité. En 2017, I'ISU a élaboré un plan compatible avec l'une des principales recommandations de la révolution des données, qui appelait à considérer les systèmes statistiques des pays comme les fondements à partir desquels mettre en œuvre ce changement et parvenir à des statistiques nationales sur l'éducation.

Dans ce contexte, l'Ed-DQAF est un outil à utiliser à l'échelle nationale, qui offre une évaluation complète de la qualité des données sur l'éducation. II devient la clé de l'identification des points forts et des points faibles du système de production de données existant dans le cadre d'un processus qui doit conduire à la conception d'une stratégie nationale à même de relever les nouveaux défis du suivi.

L'Ed-DQAF évalue le processus de production, la gestion des productions de données ainsi que les caractéristiques de l'environnement ou de l'infrastructure statistique favorable, et couvre donc toutes les phases et tous les aspects de la collecte, du traitement et de la diffusion des données. Adapté à chaque source de données sur l'éducation, le cadre mesure la qualité des données produites à l'aune des normes et pratiques internationales actuelles qui fixent les niveaux de qualité attendus. La méthodologie de l'évaluation repose sur l'hypothèse selon laquelle les statistiques nationales sur l'éducation respectent les normes statistiques en matière de qualité lorsqu'elles sont produites dans le cadre d'un processus qui satisfait aux normes et standards définis par la méthodologie de I'Ed-DQAF. L'objectif ultime du processus de production des données est de fournir des informations statistiques solides sur l'éducation qui vont permettre d'éclairer les politiques, les recherches et les processus décisionnels aux niveaux national et international.

En mai 2017, I'ISU a proposé un CdP spécifique aux statistiques sur l'éducation, composé de 12 principes relatifs à la qualité et liés à l'environnement institutionnel, aux processus de production statistique et aux productions statistiques (voir Encadré 6). Étayant la construction de l'Ed-DQAF, chaque principe comprend un ensemble d'indicateurs qui servent de référence pour un examen approfondi de la mise en œuvre (voir l'Annexe 1).

Le CdP pour les statistiques sur l'éducation a pour objectif de s'assurer que les statistiques sur l'éducation, qui sont principalement produites à des fins administratives, sont non seulement pertinentes, opportunes et exactes mais aussi qu'elles respectent les principes d'indépendance professionnelle, d'impartialité et d'objectivité. L'audience nationale cible pour le CdP est les différentes parties prenantes impliquées dans la production de statistiques sur l'éducation, principalement les ministères de l'éducation et les instituts de statistique nationaux.

Il est bénéfique d'évaluer la mise en œuvre du CdP par le biais d'un processus d'examen externe effectué par des pairs de façon périodique. Les membres de l'équipe de l'examen externe doivent être indépendants des organisations de mise en œuvre et spécialisés dans l'éducation, les statistiques et la gestion de la qualité. La portée de l'examen peut concerner l'ensemble du secteur national de l'éducation ou un ministère, une institution, ou un sous-secteur de l'éducation. 
Environnement institutionnel : Les facteurs organisationnels et institutionnels ont une influence significative sur l'efficacité et la crédibilité des ministères de l'éducation qui élaborent, produisent et diffusent les statistiques de l'éducation. Les aspects pertinents sont le cadre politique et juridique, l'adéquation des ressources, le professionnalisme, la transparence et les normes éthiques.

- Principe 1 : Cadre politique et juridique. L'environnement juridique et institutionnel régissant les statistiques de l'éducation a une influence significative sur l'efficacité et la crédibilité du ministère de l'éducation qui produit et diffuse les statistiques de l'éducation.

- Principe 2 : Adéquation des ressources. Le ministère de l'éducation s'assure que les ressources sont proportionnées aux programmes statistiques, personnels, installations, équipements, technologies, formations et financements de leurs systèmes d'information sur la gestion des établissements d'enseignement.

- Principe 3 : Sensibilisation à la qualité. La qualité est la clé de voûte des activités statistiques. Les ministères de l'éducation identifient systématiquement et régulièrement les points faibles et les points forts afin d'améliorer continuellement la qualité du processus et de la production.

- Principe 4 : Professionnalisme. Les politiques et pratiques statistiques sont guidées par des principes professionnels.

- Principe 5 : Transparence. Les ministères de l'éducation élaborent, produisent et diffusent les statistiques de l'éducation d'une façon objective et transparente, dans le cadre de laquelle les utilisateurs sont traités équitablement.

- Principe 6 : Normes éthiques. Les politiques et les pratiques sont conformes à des normes éthiques.

Source : Institut de statistique de l'UNESCO (à paraître).
Processus statistiques : Les normes, principes directeurs et bonnes pratiques internationaux sont entièrement respectés dans le cadre des processus utilisés par les ministères pour organiser, collecter, traiter et diffuser les statistiques officielles. La crédibilité des statistiques est renforcée par une réputation de bonne gestion et d'efficacité. Les aspects pertinents sont la solidité méthodologique.

- Principe 7 : Solidité de la méthodologie. La base méthodologique des statistiques de l'éducation suit les normes, principes directeurs et bonnes pratiques internationaux.

- Principe 8 : Exactitude et fiabilité. Les sources de données et les techniques statistiques sont solides et les résultats des statistiques de l'éducation brossent un portrait suffisamment fidèle de la réalité.

Résultats des statistiques sur l'éducation : Les statistiques disponibles répondent aux besoins des usagers. Les statistiques de l'éducation sont conformes aux normes internationales en matière de qualité et répondent aux besoins des institutions internationales, gouvernements et instituts de recherche, aux préoccupations du monde des affaires ainsi qu'au grand public. Les aspects importants sont la pertinence, la périodicité et le caractère opportun, la cohérence et l'accessibilité ainsi que la clarté.

- Principe 9 : Pertinence. Les statistiques de l'éducation répondent aux besoins des utilisateurs.

- Principe 10 : Périodicité et caractère opportun. Les statistiques de l'éducation sont publiées en temps utile, selon une périodicité acceptée au plan international.

- Principe 11 : Cohérence. Les statistiques de l'éducation sont cohérentes avec un ensemble de données et au fil du temps, ainsi qu'avec d'autres ensembles de données importants.

- Principe 12 : Accessibilité et clarté. Les statistiques et métadonnées sur l'éducation sont aisément disponibles, présentées d'une façon claire et compréhensible, et un soutien aux utilisateurs est disponible. 
L'Ed-DQAF sert d'outil permettant de mettre en œuvre les principes du CdP, qui sont intégrés dans l'Ed-DQAF en tant que troisième niveau de la structure, et les indicateurs en tant que quatrième niveau. La méthodologie du DQAF a été créée sur la base de trois principes régissant la qualité des statistiques de l'éducation au niveau national et est alignée sur les Principes fondamentaux des statistiques officielles de l'ONU adoptés par l'Assemblée générale des Nations Unies en 2014 et sur le NQAF de l'ONU (voir Encadré 4). Ces principes généraux sont les suivants.

- Aptitude à l'emploi. La qualité d'une information statistique est interprétée au sens large, et englobe la mesure dans laquelle les processus statistiques produisent des résultats qui satisfont aux attentes des utilisateurs. Des productions de bonne qualité sont des statistiques qui, du point de vue de l'utilisateur, sont aptes à l'emploi ou, en d'autres termes plus précis, pertinentes, exactes, fiables, cohérentes, opportunes, accessibles et interprétables. Des processus de production statistique de bonne qualité sont des processus qui utilisent des systèmes et une méthodologie solides, qui utilisent des concepts, des classifications et des méthodes reconnus au plan international, et qui sont économiques.

- Efficacité des Principes fondamentaux des statistiques officielles. L'environnement institutionnel garantit l'efficacité des Principes fondamentaux des statistiques officielles et reconnaît le caractère indispensable de l'objectivité, de l'impartialité, de la transparence et de la coordination statistique. En outre, les données personnelles collectées par des services statistiques doivent être strictement confidentielles et utilisées exclusivement à des fins statistiques.

- Sensibilisation à la qualité. Le soutien constant et sur le long terme apporté par l'équipe dirigeante est la pierre angulaire de la mise en œuvre réussie de la politique relative à l'assurance qualité. Les responsables opérationnels doivent accorder l'attention voulue au suivi de la qualité de la collecte, du traitement et de la diffusion des statistiques et s'occuper des aspects touchant à la qualité dans la planification du programme statistique. Chaque organisation doit disposer d'un système de gestion de la qualité à même d'identifier les attentes des utilisateurs et de s'assurer que tous les processus statistiques et de soutien fonctionnent conformément aux exigences auxquelles ils sont censés satisfaire (ONU, 2014).

La structure de l'Ed-DQAF est organisée en forme de cascade, allant du plus abstrait au plus concret et comportant quatre niveaux qui se chevauchent : les dimensions, les sous-dimensions, les indicateurs et les bonnes pratiques. Le niveau supérieur est composé de trois sections : l'environnement favorable, les processus statistiques et les productions de statistiques sur l'éducation. Le second niveau est la dimension de la qualité, deux pour chaque section. Chaque dimension de la qualité est alignée sur le troisième niveau et correspond à un, deux ou trois principes de la qualité du CdP. Les liens entre la structure de I'Ed-DQAF, le CdP de l'ISU et le NQAF de I'ONU sont présentés à l'Annexe 2.

\subsubsection{Utiliser les cadres d'évaluation de la qualité des données pour les statistiques nationales de l'éducation}

Pour améliorer les statistiques nationales de l'éducation, I'ISU a mis en œuvre des cadres d'évaluation de la qualité des données à deux niveaux.

L'Ed-DQAF a été conçu pour être utilisé dans le contexte du Système statistique national de l'éducation (SSNE), qui fait partie du vaste système statistique national chargé de produire des statistiques officielles à des fins d'élaboration de politiques et de planification. Le SSNE est au cœur de la collecte, du traitement, de la diffusion et de l'utilisation des données et suit par conséquent les ensembles nationaux de définitions, méthodologies, classifications et outils. Pour surveiller le secteur de l'éducation, le SSNE doit efficacement intégrer différentes sources de données, y compris des ensembles de données administratives (collectées et stockées conformément au Système d'information et de gestion de l'éducation [SIGE]), des enquêtes auprès des ménages, des évaluations des apprentissages ainsi que des ensembles de données sur les financements et dépenses. Cependant, de nombreux pays ne disposent pas de systèmes bien établis qui intègrent ces différentes sources de données (ISU, 2016).

Dans de nombreux pays, et cela quel que soit le niveau de leur développement, la coordination des systèmes statistiques parallèles et fragmentés en place est l'un des plus grands défis à relever dans l'élaboration de données relatives à l'éducation de 


\section{Figure 4. Structure de l'Ed-DQAF à des fins administratives}

\section{Environnement}

L'environnement institutionnel garantit l'efficacité des principes fondamentaux des statistiques officielles.

Principes 1 à $3-9$ indicateurs Le principe d'objectivité de la collecte, du traitement et de la diffusion des statistiques est strictement respecté. Principes 4 à $6-11$ indicateurs

La base méthodologique des statistiques suit les normes, principes directeurs et bonnes pratiques acceptés au plan international. Principe 7-4 indicateurs

Les sources des données et les techniques statistiques sont solides et les productions statistiques brossent un portrait suffisamment fidèle de la réalité. Principe $8-5$ indicateurs

Les statistiques sont pertinentes, publiées en temps utile et régulièrement; elles sont cohérentes et suivent une politique de révisions. Principes 9 à $11-9$ indicateurs

Les données et les métadonnées sont aisément disponibles et le soutien aux utilisateurs est approprié. Principe $12-3$ indicateurs

\section{Pré-requis de qualité}

Intégrité

Précision et fiabilité

\section{Résultats}

statistiques de

I'éducation

Facilité de service

Accessibilité favorable
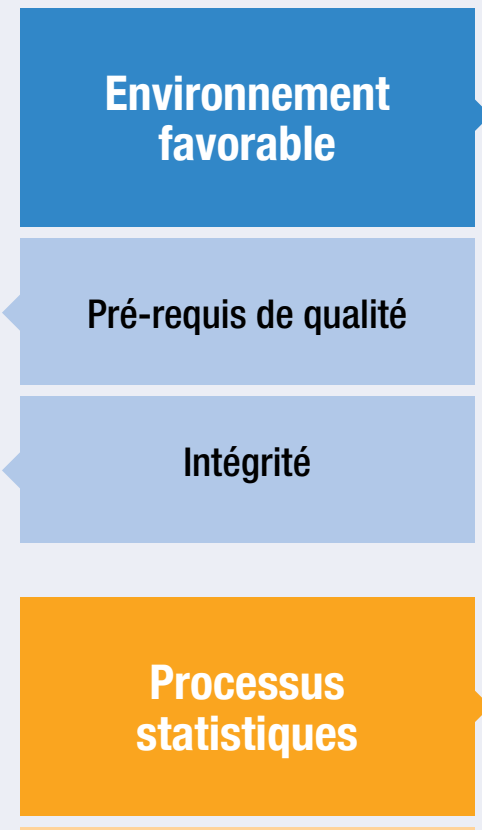

La solidité méthodologique

Les normes, principes directeurs et bonnes pratiques internationaux sont entièrement respectés dans les processus utilisés par les ministères pour organiser, collecter, traiter et diffuser les statistiques officielles. La crédibilité des statistiques est renforcée par une réputation de bonne gestion et d'efficacité. Cela comprend la solidité de la méthodologie, l'exactitude et la fiabilité.
Les statistiques disponibles répondent aux besoins des utilisateurs. Les statistiques de l'éducation respectent les normes internationales en matière de qualité et répondent aux besoins des institutions internationales, gouvernements, instituts de recherche, des entreprises et du grand public. Les aspects importants concernent la pertinence, la périodicité et la publication en temps utile, la cohérence, ainsi que l'accessibilité et la clarté. 
qualité. Le suivi des systèmes éducatifs relève de la responsabilité de multiples ministères, agences et services à différents niveaux de gouvernement, à l'image de la structure du système éducatif. Dans plusieurs pays, la fonction statistique est considérablement décentralisée, et les statistiques sont produites indépendamment dans un certain nombre d'unités organisationnelles au sein desquelles aucun rôle de coordination significatif n'est assigné et sans l'appui d'un SIGE central en mesure de fédérer les différents systèmes d'information et de gestion dans les ministères ou les agences. Les activités de suivi des statistiques ont par ailleurs tendance à calquer l'organisation des activités éducatives, et sont organisées et groupées autour des principaux soussecteurs de l'éducation : petite enfance, primaire, secondaire, formation professionnelle, postsecondaire non tertiaire et supérieur.

Pour faciliter l'évaluation de la qualité, les activités de production statistique exercées par les entités de collecte des données peuvent être envisagées comme des processus de production statistique autonomes et distincts, dont chacun :

- est sous le contrôle d'une seule unité ;

- couvre un ou plusieurs sous-secteurs de l'éducation, ou un sujet spécifique (financement, évaluation de l'apprentissage, enseignants, etc.) ou un ensemble de sujets liés à l'éducation ;

- acquiert des données auprès d'une source ou d'un ensemble de sources spécifiques ;

- réalise un produit statistique spécifique ou un ensemble de produits connexes.

Quel que soit le niveau de coordination, l'objectif commun de l'organisation membre du SSNE doit être l'harmonisation des statistiques de l'éducation produites par le SSNE, c'est-à-dire que ces statistiques devraient emprunter, dans la mesure du possible, des concepts, des classifications et des définitions communs et que les données produites devraient être mutuellement compatibles. La coordination est facilitée lorsque les membres partagent la même interprétation des questions relatives à la qualité et qu'ils se réfèrent à un ensemble de principes communs et à un cadre d'assurance de la qualité commun pour organiser leurs activités statistiques.
À cette fin, I'ISU recommande que chaque organisation produisant des données pertinentes sur le secteur de l'éducation adopte officiellement les 12 principes de la qualité tels que présentés dans le CdP pour les statistiques de l'éducation. De leur côté, ces organisations doivent présenter le CdP et conduire les unités responsables des statistiques de l'éducation à mettre en œuvre le CdP et à se référer à l'Ed-DQAF pour surveiller la qualité de leur production statistique. L'Ed-DQAF utilisé au niveau national intègre un ensemble d'indicateurs de la qualité pour chaque principe du CdP, qui doit être utilisé lors de la conception de nouvelles activités, de la réorganisation de processus et du suivi ou de l'évaluation des processus.

Le cadre a pour objet d'assurer la qualité lors de la conception ou de la refonte d'un processus statistique et lors de l'évaluation des performances de qualité d'un processus statistique en cours d'utilisation. À ces deux fins, il est nécessaire de documenter le DQAF générique sur les objectifs du processus en spécifiant la base méthodologique (Principe 7), les sources et techniques (Principe 8), la satisfaction de l'utilisateur (Principe 9) ainsi que la périodicité et la ponctualité prévues (Principe 10). En outre, le personnel responsable du processus d'évaluation a besoin d'un manuel comprenant des lignes directrices générales pour chaque principe et liées à chaque indicateur, des détails sur les bonnes pratiques de la mise en œuvre, des instructions, méthodes et procédures, ainsi que des mécanismes de suivi de la qualité.

Deux types d'évaluation peuvent être effectués à l'aide du cadre documenté : l'auto-évaluation de la qualité et l'évaluation de la qualité externe.

- Une auto-évaluation de la qualité est conduite par le personnel responsable du processus statistique, au moins une fois par an ; ses objectifs consistent à aider le personnel responsable à brosser un portrait de la qualité de ses processus et produits, et donc à identifier les faiblesses structurelles et à proposer des améliorations de la qualité.

- Pour les évaluations externes, les objectifs sont similaires et principalement focalisés sur la mise en œuvre effective des lignes directrices de la qualité utilisées. Contrairement aux auto- 
évaluations, les membres de l'équipe d'évaluation (ou pairs) ne sont pas impliqués dans l'exécution du processus statistique examiné, mais ils doivent être familiarisés avec l'assurance qualité de la production des statistiques de l'éducation ou d'autres domaines.

Les deux exercices d'évaluation exigent l'organisation de réunions sur la mise en œuvre et le suivi avec le personnel responsable de tous les aspects du traitement, les principaux utilisateurs des données et les fournisseurs de données fondamentaux. Les participants peuvent surveiller les mesures prises pour répondre aux recommandations émises lors des précédentes évaluations. L'utilisation d'une liste de contrôle établie à partir des bonnes pratiques facilite cet exercice d'évaluation; une échelle peut être utilisée pour évaluer le degré de conformité pour chaque pratique. L'Encadré 7 donne un exemple de mesures utilisées par l'ISU pour évaluer des statistiques nationales de l'éducation.

\section{Encadré 7. Listes de contrôle utilisées pendant les évaluations de la qualité : l'exemple du}

\section{Principe 8}

Principe 8 - Exactitude et fiabilité. Les sources de données et les techniques statistiques sont solides et les résultats des statistiques sur l'éducation brossent un portrait suffisamment fidèle de la réalité.

Indicateur 8.1 : Les sources de données disponibles constituent une base appropriée pour compiler les statistiques. compilar estadísticas.

Lors des évaluations externes du Principe 8, les équipes de I'ISU ont utilisé la liste de contrôle des bonnes pratiques suivante pour évaluer les mesures de l'Indicateur 8.1.

- Un exercice administratif annuel de collecte de données recueille des informations sur la structure du système éducatif, les élèves, les enseignants et les examens.

- La couverture est complète en termes géographiques (locale, régionale, centrale).

- La couverture est complète en termes de sous-groupes des unités de collecte pertinents (par exemple, élèves et enseignants de sexe masculin et féminin, écoles publiques et privées, enseignants formés et non formés, élèves et enseignants à plein temps et à temps partiel).
- Les procédures de maintenance des listes d'écoles sont appropriées (doublons, confusion des noms, solidité des codes administratifs, autres divergences remarquées).

- La présentation des données sur l'âge est fiable.

Le personnel de I'ISU utilise une échelle de 1 à 4 pour évaluer la conformité à chaque pratique établie :

Pratique non observée (notée 1)

Pratique largement non observée (notée 2)

Pratique largement observée (notée 3)

Pratique observée (notée 4) 


\subsection{Produire des indicateurs de I'ODD 4 de qualité pour le suivi mondial}

Comme indiqué précédemment, la production de données et d'indicateurs internationaux de grande qualité dépend dans une très large mesure de la qualité des données collectées au niveau national. Les organisations internationales, telles que I'ISU et la DSNU, et les organisations régionales, telles qu'Eurostat, jouent un rôle fondamental car elles assurent la production de données et d'indicateurs transnationaux de qualité. Agissant fréquemment comme des institutions coordinatrices rassemblant les parties prenantes pour qu'elles élaborent les données transnationales, les organisations internationales jouent un rôle essentiel dans l'élaboration des normes, la compilation des statistiques et l'établissement des rapports sur les résultats concernant les principales questions internationales.

Bien que les organisations internationales suivent des principes de collecte de données similaires à ceux des instituts nationaux de la statistique, elles sont confrontées à des défis différents. D'abord, les instituts nationaux ne sont pas tenus de communiquer les données ; la publication de statistiques internationales se fait sur une base volontaire. Un autre défi est l'incertitude entourant la qualité des données primaires produites au niveau national. Les données sont généralement soumises sous une forme agrégée et les organisations internationales n'exercent aucune influence ou aucun contrôle sur le processus de production (autre que fournir des méthodologies et des normes et, dans certains cas, renforcer les capacités).

En tant qu'institut officiel de la statistique de I'UNESCO, I'ISU a pour mission de collecter les statistiques de l'éducation partout dans le monde. Son rôle d'institution dépositaire en fait également le responsable de la collecte de la plupart des indicateurs de l'éducation de l'ODD 411. L'ISU élabore des méthodes, des normes et des processus et apporte une assistance technique aux États membres et aux autres organisations internationales ou régionales impliquées dans la production de données sur l'éducation.
L'ISU joue un rôle vital au sein de la communauté mondiale de l'éducation car elle exerce de multiples activités qui fournissent des données de qualité et comparables entre les différents systèmes éducatifs et pays. II a notamment la responsabilité de diffuser des normes internationales, telles que la Classification internationale type de l'éducation (CITÉ), de définir et d'établir une méthodologie comparable pour l'élaboration des indicateurs, de compiler et de diffuser des statistiques internationales sur l'éducation grâce aux collectes de données effectuées annuellement auprès des États membres ou des organisations internationales et d'apporter un soutien technique aux États membres.

Le programme de travail statistique actuel de I'ISU s'articule autour des trois piliers décrits dans la Stratégie de I'ISU à moyen terme pour 2017-2021 (voir Figure 5). Le programme de travail est régi par un cadre d'établissement des rapports et de suivi de la qualité qui oriente les activités de production de données internes. II repose sur le Cadre d'évaluation de la qualité des données du FMI (DQAF), les éléments de la Définition de la qualité Eurostat, les Principes de l'ONU régissant les activités statistiques internationales, les Principes fondamentaux des statistiques officielles et les Méthodes et outils d'évaluation de la qualité des données (Assemblée générale des Nations Unies, 2014 ; DSNU, 2013).

Produire la base de données la plus complète du monde sur les statistiques de l'éducation, tout en établissant des rapports sur les nouveaux indicateurs de l'ODD 4, est une tâche complexe qui comporte de multiples aspects, parties prenantes, processus et sources de données. La Figure 6 résume le processus de production de données et l'interaction entre les autorités statistiques nationales, les organisations régionales et les institutions dépositaires de données pour produire des données et des indicateurs internationaux de qualité sur l'éducation. Les données sur l'éducation des systèmes statistiques nationaux ou des organisations régionales, composées de données et de métadonnées collectées au niveau national, sont communiquées à l'ISU, qui est responsable de la production d'indicateurs comparables au plan transnational, du calcul des agrégats mondiaux et régionaux et de la diffusion des données et métadonnées qui en résultent par le biais de mécanismes de diffusion et de suivi.

\footnotetext{
${ }^{11}$ EI UIS también tiene a su cargo la recolección de datos para diversos programas de la UNESC0, entre otros de ciencia, tecnología, cultura y comunicación.
} 


\section{Figure 5. La Stratégie de l'ISU à moyen terme pour 2017-2021}

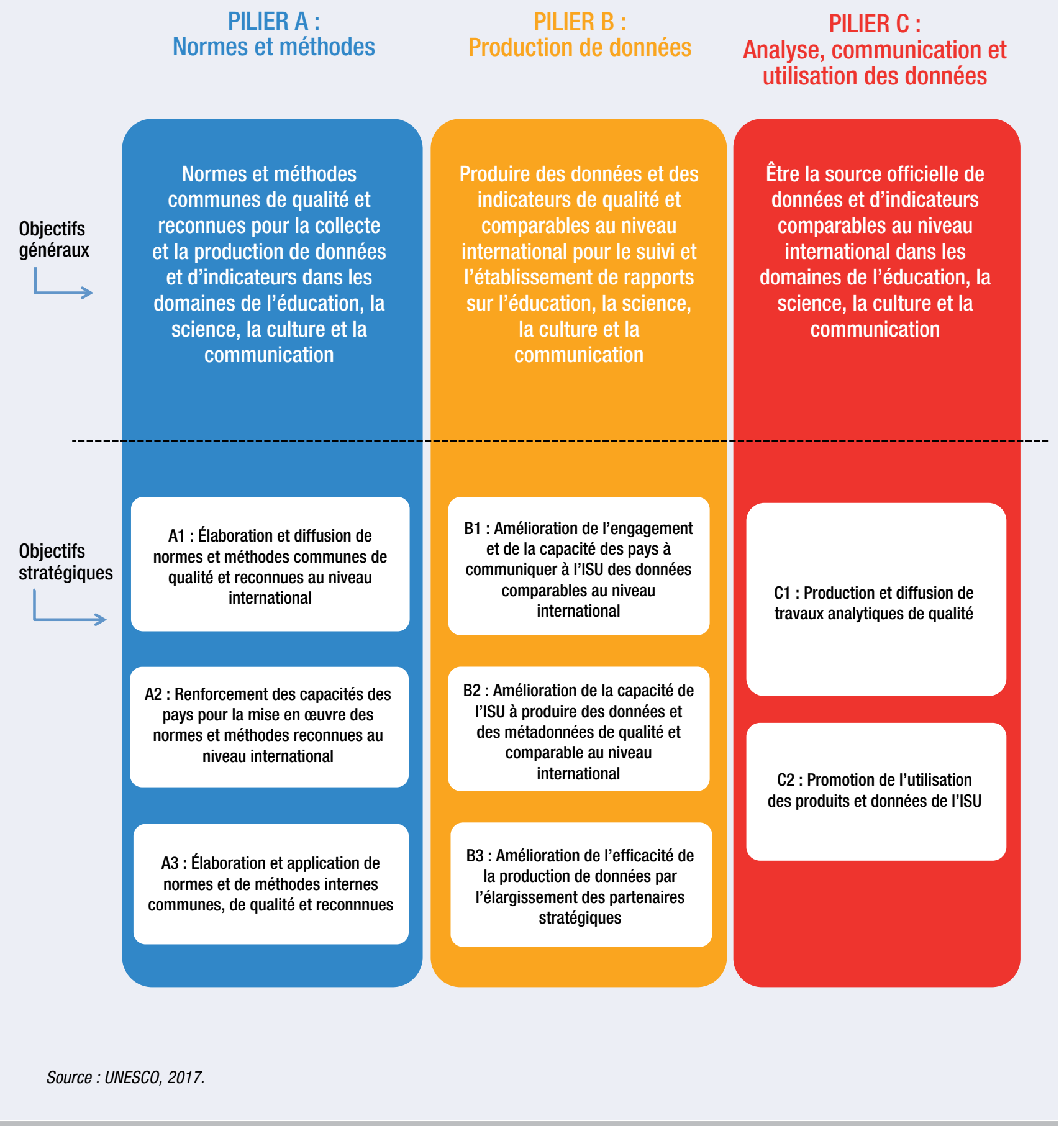




\section{Figure 6. Flux de données pour produire les indicateurs mondiaux de l'ODD 4}

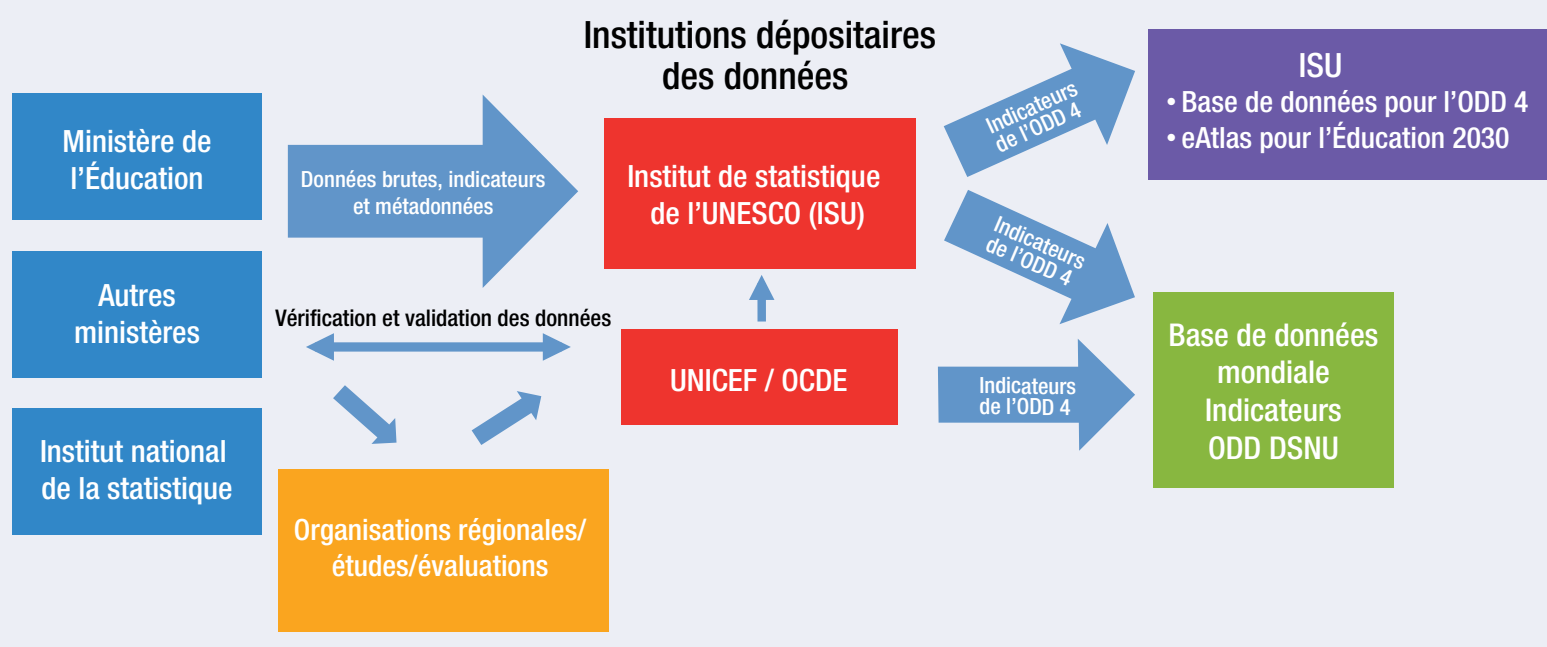

Intégrer et harmoniser les flux de données et les indicateurs exige de mettre en œuvre au niveau international des processus systématiques et méthodiques. Les flux de données des organisations nationales, régionales et internationales sont bien définis et reposent sur de solides principes afin de permettre d'obtenir des résultats comparables au plan international, mais qui sont également représentatifs au plan national. L'ISU travaille avec des organismes régionaux Eurostat, CEPALC et OCDE - afin d'élaborer et de diffuser des méthodologies et normes communes pour éliminer les doublons et faire en sorte que les données soient recueillies de la façon la plus économique possible.

Les ODD 4 sont de nature complexe et la plupart exige la conception de nouvelles méthodologies, définitions et méthodes de calcul et que soient apportées des modifications significatives au système statistique, tant au plan national qu'international. Le flux de données représenté à la Figure 6 occulte les difficultés liées aux activités qu'il faut surmonter à chacune des étapes du processus de production pour transmettre des statistiques internationales de qualité. Les trois phases principales - définition des normes, production des données et diffusion des données sont décrites ci-après.

\subsection{1 Élaboration de définitions et de normes internationales en vue de la production des indicateurs de l'ODD 4}

Collecter des données auprès de sources nationales ou régionales dans le but d'établir des rapports internationaux exige de définir les bases solides de la production de données sur l'éducation fiables et comparables. Comme les statistiques nationales, les statistiques internationales exigent des cadres robustes qui vont garantir la grande qualité des résultats et processus. Les principes sous-jacents sont tout à fait similaires, mais des complexités différentes interviennent au niveau mondial en raison de la coordination des pays, territoires, institutions et gouvernements.

En premier lieu, l'élaboration des normes, méthodologies, définitions et classifications acceptées au plan international doit assurer la compatibilité des données entre les pays, régions et périodes.

La comparaison de systèmes éducatifs nationaux et de leurs résultats exige un cadre normalisé permettant d'aligner les systèmes éducatifs nationaux avec les définitions internationales. L'un des outils les plus importants pour atteindre cet 
objectif est la CITÉ, produite par l'ISU, qui fournit un cadre pour classer les programmes éducatifs et les niveaux d'éducation conformément aux catégories convenues au plan international (voir Encadré 8). L'ISU et ses partenaires appliquent les normes et méthodologies de la CITÉ afin de s'assurer que les données nationales sont précises et comparables au plan international.

\section{L'implication de l'ensemble des parties} prenantes - notamment le gouvernement, le secteur privé et les organisations internationales et non gouvernementales - dans l'énonciation des définitions et normes de la production de statistiques internationales sur l'éducation est indispensable à la production de résultats de grande qualité. L'ISU élabore les définitions et les normes de l'éducation en étroite collaboration avec les États membres et les organisations internationales et régionales impliquées dans les activités liées à l'éducation. L'ISU promeut cette collaboration en travaillant avec des organisations régionales - telles que Eurostat et l'OCDE - dont les États membres sont également impliqués dans les élaborations méthodologiques. Les trois organisations collectent les données sur l'éducation dans le cadre d'un effort conjoint, en utilisant des questions, normes, méthodologies et outils communs, ce qui réduit le fardeau des systèmes statistiques nationaux et internationaux.
En 2014, Eurostat, l'OCDE et l'ISU ont mis en œuvre l'Échange de données et de métadonnées statistiques (SDMX), qui consiste à intégrer des normes techniques, des directives statistiques ainsi qu'une architecture informatique et des outils dans leurs collectes de données respectives. La collaboration grâce au SDMX et la création d'une définition commune de la structure des données, comme convenu au plan international, a des avantages conséquents : fournir un mécanisme qui assure la cohérence des données entre les organisations internationales et qui améliore l'efficacité des échanges de données et des processus de gestion des données tout au long du cycle de vie des données statistiques.

En ce qui concerne I'ODD 4, I'ISU a réalisé plusieurs consultations et a convoqué des experts du monde entier, par l'intermédiaire de l'Alliance mondiale pour le suivi de l'apprentissage (GAML) et du Groupe de coopération technique (GdCT), afin d'élaborer des définitions conceptuelles bien définies et une méthodologie normalisée pour chacun des indicateurs mondiaux et thématiques proposés. La méthodologie ainsi obtenue pour les 43 indicateurs a été publiée dans Metadata for the Global and Thematic Indicators for the Follow Up and Review of ODD 4 and Education 2030, qui fournit, pour chaque indicateur, une définition, sa finalité, la méthode de calcul, l'interprétation, les sources de données utilisées pour son calcul, la ventilation requise et les limites (ISU, 2017e).

\section{Encadré 8. La Classification internationale type de l'éducation (CITÉ)}

La CITÉ est un système de classification qui fournit un cadre en vue de la description statistique complète d'un système éducatif national et une méthodologie qui traduit les programmes éducatifs et les qualifications sur lesquelles ils débouchent en niveaux d'éducation comparables au plan international.

La CITÉ a été créée en 1976 par les États membres de I'UNESCO pour faciliter les comparaisons entre pays et mesurer les progrès accomplis sur la voie des objectifs internationaux en matière d'éducation. La structure des systèmes éducatifs nationaux varie de façon significative entre les pays, par exemple pour définir l'âge officiel d'admission, la durée de chacun des niveaux d'éducation et la nomenclature utilisée pour les niveaux. Elle donne la possibilité effective d'inscrire tout système éducatif national dans un cadre comparable au plan international. Ce processus de cartographie rend les données sur les élèves et les taux nets d'inscription dans le primaire, par exemple, comparables entre les pays. La version la plus récente de la CITÉ a été adoptée en 2011 et la Classification des domaines d'éducation et de formation (CITÉ-F 2013) dont elle est assortie a été adoptée en 2013. 


\subsubsection{Collecte des données, traitement et validation}

L'ISU compile les données et métadonnées liées à l'éducation recueillies auprès de différentes sources aux niveaux national, régional et international. Bien que la plupart des données soient collectées par l'intermédiaire d'enquêtes annuelles de l'ISU envoyées aux États membres, l'Institut produit également des indicateurs qui reposent sur des enquêtes réalisées auprès des ménages et des évaluations internationales des apprentissages comme décrit ci-après.

- Enquêtes annuelles. L'ISU conduit deux enquêtes mondiales sur l'éducation, sur les programmes éducatifs officiels et sur l'alphabétisation et les niveaux d'éducation, qui impliquent les instituts de statistique nationaux ou les ministères de l'Éducation, selon les modalités du protocole officiel ${ }^{11}$. Ces enquêtes consistent en un ensemble de questionnaires sur les programmes éducatifs, les élèves, les ressources financières et humaines, l'alphabétisation et les niveaux d'instruction, qui reposent sur des normes internationales afin d'assurer la comparabilité entre les pays. Les principales sources de données sont les dossiers administratifs des études menées dans les écoles ou les données agrégées des enquêtes sur la main-d'œuvre ou les ménages. Ces enquêtes sont la source de plusieurs des indicateurs de l'ODD 4.

- Enquêtes auprès des ménages. L'ISU compile et diffuse des données d'enquêtes sur les ménages afin de produire des indicateurs permettant d'examiner différentes caractéristiques de populations spécifiques, qui ne sont généralement disponibles que par l'intermédiaire de ces sources. L'ISU rassemble des ensembles de données issues d'enquêtes sur les ménages auprès, par exemple, de l'Enquête démographique et de santé (DHS) et de l'Enquête par grappes à indicateurs multiples (MICS), ou de recueils de données conservés par des organisations telles que le CEPALC, le projet Integrated Public Use Microdata Series International (IPUMS) et la Banque mondiale. Les données sont exploitées pour calculer différents indicateurs de l'éducation, tels que les taux d'assiduité et d'achèvement.

\section{- Évaluations des apprentissages. L'ISU a} identifié neuf évaluations des apprentissages transnationales qui satisfont aux critères requis pour mesurer l'Indicateur 4.1.1 de l'ODD 4 (LaNA, PASEC, PILNA, PIRLS, PISA, SACMEQ, SEA PLM, TERCE et TIMSS), a accédé à ces sources de données et a commencé à calculer certains indicateurs clés liés aux résultats d'apprentissage pour l'ODD $4^{12}$.

Les données peuvent être obtenues directement en les extrayant d'une base de données ou en procédant à une collecte de données. Pour l'extraction des données, un accord officiel est conclu avec les organisations concernées et, pour procéder à une collecte de données, les normes sont intégrées dans les différents instruments de collecte des données (par exemple les questionnaires), qui sont mis à jour de façon à comprendre les saisies de données nécessaires pour calculer les indicateurs de l'ODD ainsi que les instructions et définitions détaillées.

Une fois que les données ont été recueillies, elles subissent une série de contrôles de la qualité (voir Figure 7). Les analystes de données de I'ISU examinent les données afin d'assurer la couverture de l'intégralité du système éducatif national et la conformité avec les normes et définitions internationales. Les analystes comparent également, si possible, les données de plusieurs sources, telles que les données des enquêtes auprès des ménages et toute série chronologique et annuaire ou base de données statistiques disponible. Les données communiquées sont également comparées avec celles d'autres pays de la même région ou de la même tranche de revenu ${ }^{13}$.

11 Les États membres de l'Union européenne ou de l'OCDE prennent part à l'enquête ISU-OCDE-Eurostat (IOE).

12 Ce sont l'Évaluation des capacités en lecture, écriture et calcul (LaNA), le Programme d'analyse des systèmes éducatifs de la CONFEMEN (PASEC), I'Évaluation des capacités en lecture, écriture et calcul des îles du Pacifique (PILNA), le Programme international de recherche en lecture scolaire (PIRLS), le Programme for International Student Assessment (PISA), le Consortium d'Afrique australe pour le pilotage de la qualité de l'éducation (SACMEQ), les Principaux indicateurs d'apprentissage en Asie du Sud-Est (SEA PLM), le Tercer Estudio Regional Comparativo y Explicativo (TERCE) et les Tendances dans les études internationales de mathématiques et de sciences (TIMSS).14 Véase UIS 2017j, para obtener información adicional sobre el cálculo de indicadores nacionales y regionales.

${ }^{13}$ Pour en savoir plus sur le calcul des indicateurs nationaux et régionaux, voir ISU (2017j). 


\section{Figure 7. Assurance de la qualité lors de la collecte, du traitement et de la validation effectués par l'ISU}

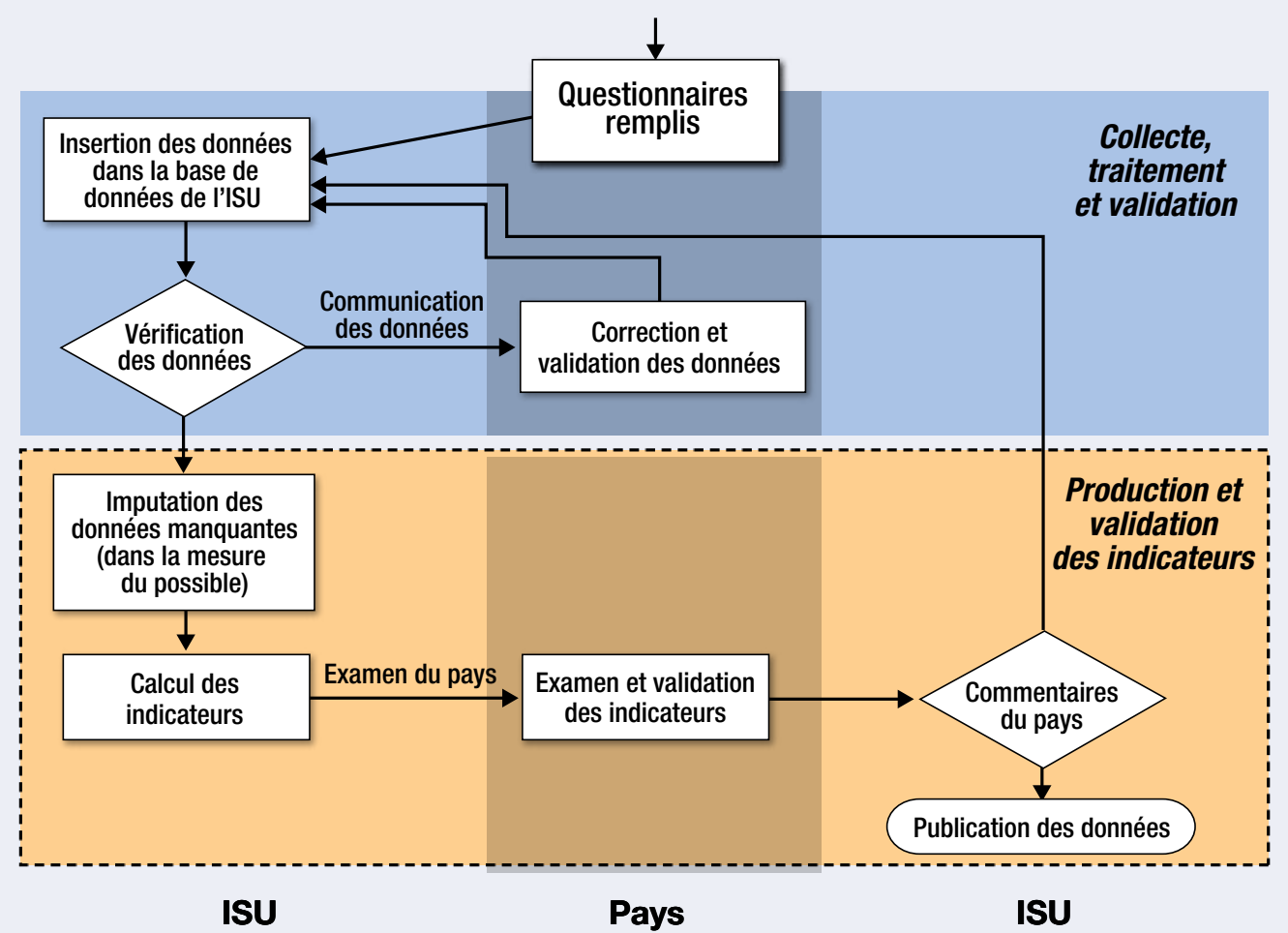

Source : Institut de statistique de l'UNESCO, 2017j.

Pour les données administratives, le processus de validation des données exige de documenter les disparités ou problèmes de données et d'envoyer des questions aux autorités nationales afin qu'elles y répondent. Ce processus d'examen implique fréquemment des commentaires supplémentaires de la part des États membres ainsi qu'un nouveau traitement des données. Après l'examen final, le personnel de l'ISU confirme les données en utilisant les contributions des répondants et en prenant des décisions concernant la qualité des données. Si la qualité des données est considérée comme insuffisante, les décisions peuvent consister à supprimer les données ou à les remplacer par une estimation ou une autre source susceptible de permettre la production d'indicateurs améliorés. Des estimations sont également produites lorsque des données sont manquantes ou erronées. L'ISU produit des estimations qui reposent sur les données existantes de l'enquête actuelle ou précédente, des publications statistiques ou des bases de données nationales et d'autres sources de données fiables (par exemple, des enquêtes auprès des ménages).
Le processus de production des données et indicateurs de l'ISU est transparent et a pour objectif d'impliquer les autorités nationales afin que les données soient complètes et exactes. Les États membres sont encouragés à effectuer leurs propres estimations, dans le respect des normes internationales. Les autorités nationales sont responsables de la validation finale des données et des indicateurs, y compris des indicateurs de l'ODD 4 et des séries chronologiques complètes.

Le processus de validation est similaire pour les données provenant d'enquêtes auprès des ménages ou d'évaluations internationales, si ce n'est que le personnel contacte l'organisation où les bases de données sont conservées lorsqu'il y a des problèmes de données.

Alors que la compilation des données d'enquêtes et de recensements comporte plusieurs défis liés à l'intégration et à l'harmonisation des données, assurer la comparabilité des résultats d'apprentissage est particulièrement difficile en raison de la diversité des 
évaluations utilisées par les pays et les organisations internationales et régionales. Ces difficultés sont liées aux différences entre les trois définitions clés qu'établissent ces évaluations : premièrement, qui faut-il et que faut-il évaluer (cadre conceptuel), deuxièmement, comment faut-il évaluer (cadre méthodologique) et enfin, comment communiquer les résultats (cadre du rapport) (ISU, 2017h). Chacune de ces phases doit être soigneusement examinée afin que la stratégie conçue produise des indicateurs de l'apprentissage adaptés à l'ODD 4 - Cadre d'action Éducation 2030 (voir Encadré 9).

\section{Encadré 9. Défis de la production des indicateurs des résultats d'apprentissage}

Dans le domaine de la production des indicateurs des résultats d'apprentissage reposant sur des bases comparables au plan international, il faut relever trois défis principaux. Premièrement, des données nationales, régionales et mondiales sur les compétences de base en littératie et en numératie sont fréquemment collectées, mais ne peuvent être utilisées d'une façon intégrée pour brosser un tableau global de l'apprentissage. Deuxièmement, si des données nationales et régionales doivent être utilisées pour documenter le suivi mondial de l'apprentissage, des normes techniques partagées doivent être élaborées pour que les données soient d'une qualité similaire.

Troisièmement, les solutions doivent tenir compte de multiples points de vue : identifier des domaines d'apprentissage pertinents au plan mondial qui peuvent et qui doivent être mesurés dans le monde entier ; conceptualiser la façon dont les données nationales et régionales peuvent contribuer à documenter l'évaluation mondiale de l'éducation; et enfin, trouver un équilibre approprié entre les compétences mondiales et le rôle des influences et buts locaux sur l'éducation. II sera fondamental que les parties prenantes comprennent ce qui peut être défini, au niveau mondial, par la mesure, et ce qui doit continuer à relever du pouvoir décisionnel local.

Sous la coordination de l'ISU, les organisations qui participent à l'Alliance mondiale pour le suivi de l'apprentissage (GAML) travaillent ensemble pour élaborer :

- les indicateurs mondiaux et thématiques de l'ODD 4 liés à l'apprentissage et aux compétences ;

- les normes, principes directeurs et outils qui aident les pays à renforcer la mise en œuvre de leurs évaluations et à évaluer leur qualité ; et
- les outils de renforcement des capacités qui aident les pays à collecter, à analyser et à utiliser les données d'évaluation des apprentissages.

Ces travaux d'élaboration ont permis de franchir plusieurs étapes en 2017. Du point de vue de la disponibilité des indicateurs, il a été possible de publier les premières estimations mondiales et régionales des enfants et adolescents qui n'apprennent pas, une réponse concrète à l'appel lancé pour produire des données pour l'indicateur 4.1.1. Du point de vue de l'élaboration de méthodologies, il y a eu des accords importants sur :

- l'organisation de l'établissement d'un rapport provisoire sur l'Indicateur 4.1.1 d'une façon cohérente sur le plan international, pour la période 2017-2019;

- la mobilisation de toutes les données existantes afin d'augmenter au maximum le nombre de pays communiquant des informations, notamment les données provenant d'évaluations transnationales, mais aussi celles d'évaluations nationales qui fournissent les informations exactes nécessaires aux indicateurs ;

- la poursuite de l'élaboration des échelles de communication des données à l'ISU, qui permettent aux pays de sélectionner différentes options d'évaluation et de définir des objectifs de progrès en fonction du programme qu'ils choisissent pour la communication des données sur l'Indicateur 4.1.1, tout en permettant une certaine harmonisation des résultats; et

- l'élargissement de la comparabilité et de la couverture des évaluations des résultats d'apprentissage, par la conception de procédures permettant d'établir des liens entre les différentes évaluations régionales et internationales. 
Lorsque le processus de validation est achevé, I'ISU peut finaliser le processus de production avant de publier les indicateurs comparables au plan international. Certains indicateurs exigent que l'ISU intègre des données provenant d'autres sources internationales, comme c'est le cas des estimations et projections de la population nationale utilisées pour les taux nets de scolarisation, et qui sont obtenues auprès de la Division de la population des Nations Unies à l'UNDESA. Les données financières, telles que le produit intérieur brut (PIB) et les dépenses publiques totales sont obtenues auprès du Fonds monétaire international (FMI), de la DSNU et de la Banque mondiale. L'ISU calcule également les

agrégats reposant sur les classifications de pays par région, sous-région et revenu.

\subsubsection{Diffusion}

Une fois que la phase de production des données est achevée, I'ISU a pour mission de rendre les données relatives à l'éducation largement disponibles, sans frais, auprès des clients et utilisateurs de I'ISU. La diffusion des indicateurs de l'ODD 4 - ainsi que des autres indicateurs de l'éducation - crée le lien nécessaire entre les données et les prises de décisions fondées sur des preuves. Les données sont souvent utilisées pour évaluer des tendances passées et actuelles dans l'éducation, préparer des prévisions de futures tendances, orienter la planification politique nationale et faire des analyses thématiques des systèmes et politiques d'éducation. Grâce à la mise à disposition de données de qualité sur l'éducation au plan international, il est possible d'utiliser les comparaisons transnationales et les tendances régionales pour mesurer les progrès réalisés sur la voie de l'ODD 4 et de ses cibles.

Les productions de données doivent être aisément compréhensibles et englober toutes les métadonnées pertinentes pour assurer la clarté. En communiquant avec les utilisateurs de données pour s'assurer de la pertinence des collectes de données et faire en sorte que ses données soient aisément accessibles, I'ISU réduit la charge de communications des États membres.

L'ISU s'est focalisé sur plusieurs mécanismes de diffusion afin d'avoir un impact positif sur la disponibilité des données sur l'éducation liées à l'ODD 4 auprès des décideurs et des analystes.
- Partager les données de l'ODD 4. Bien que l'ISU publie régulièrement en ligne des données sur l'éducation dans sa base de données, le site Web de I'ISU dispose également de deux plateformes dédiées à la publication des données de l'ODD 414: l'eAtlas pour l'Éducation 2030 et le microsite ODD 4 . Lancé en 2016, l'eAtlas est un outil qui présente les indicateurs mondiaux et thématiques sur des cartes créées par l'utilisateur. Les cartes peuvent être téléchargées, ainsi que les données des tableaux utilisés pour les créer. Le microsite ODD 4 montre les dernières initiatives, analyses et tendances produites par I'ISU sur l'éducation. L'un des objectifs de la Stratégie à moyen terme est d'améliorer les liens entre les données, le glossaire et les méthodologies de l'ISU et d'améliorer I'interface utilisateur de la base de données de l'ISU.

- Analyser les indicateurs de l'ODD 4. Outre les outils de partage et les documents méthodologiques, I'ISU produit, au moyen des données, une collection de rapports analytiques sur des thèmes spécifiques liés à I'ODD $4^{15}$. L'ISU fournit également au RMSE des données officielles sur l'ODD 4 aux fins d'analyse des tendances mondiales et régionales dans cette publication annuelle, qui a pour mission de surveiller les objectifs de l'éducation. Les RMSE 2017 et 2018 comprennent respectivement des analyses liées à la responsabilité et à la transparence en éducation et aux migrations et déplacements.

\section{- Incorporer les indicateurs mondiaux dans la base} de données des Nations Unies. L'ISU propose ses données et méthodologies relatives à l'éducation (ODD 4 seulement) à la Base de données mondiale des indicateurs ODD de la DSNU ${ }^{16}$. En participant à ce processus, l'ISU s'assure que les données sur l'éducation sont à la disposition de spécialistes de domaines autres que l'éducation et qu'elles sont intégrées dans la réflexion mondiale sur les ODD.

Au-delà de ces voies de diffusion liées aux ODD, les données de l'ISU sont publiées dans des rapports et utilisées à des fins analytiques ou autres par des organisations régionales et internationales. Parmi celles-ci figurent le Programme des Nations pour le développement (PNUD), l'UNICEF et la Banque mondiale. Le Partenariat mondial pour l'éducation (GPE) s'appuie également sur les données relatives à l'éducation de l'ISU pour enrichir son agenda international de l'éducation.

\footnotetext{
${ }^{14}$ Respectivement disponibles aux adresses suivantes : http://on.unesco.org/ODD4-map, http://uis.unesco.org/en/topic/sustainabledevelopment-goal-4 et http://data.uis.unesco.org.

${ }^{15}$ Voir, par exemple, le rapport de l'ISU sur la crise mondiale de l'apprentissage, ISU (2017f).

17 https://unstats.un.org/ODDs/indicators/database/
} 


\section{Soutenir le développement des capacités statistiques nationales afin de surveiller l'ODD 4}

Conformément au mandat de l'ISU, qui consiste à collecter, compiler et diffuser des données comparables au plan international, le développement des capacités statistiques implique de s'assurer que les pays comprennent l'importance de la comparabilité et qu'ils tiennent leurs engagements à maintenir la qualité des données. Par conséquent, l'ISU doit s'assurer, dans ses activités de soutien, que les États membres:

- comprennent clairement les exigences en matière de données, les indicateurs, les méthodologies et les sources de données afin de produire des données de qualité qui pourront être utilisées pour la surveillance nationale et internationale ;

- créent un solide mécanisme, au niveau du pays, dont le mandat précis est de collecter des données de qualité auprès de différentes sources ;

- disposent de données publiables de façon à ce qu'elles puissent être utilisées par les décideurs et les parties prenantes.

Compte tenu de la complexité des données exigées pour l'ODD 4 et de la nécessité d'améliorer leur disponibilité et leur qualité, la mise en œuvre d'un cadre de données s'articulant autour de la qualité et qui soit en mesure de surveiller l'ODD 4 constituera un défi à relever par les pays, quel que soit leur niveau de développement. L'ISU est pleinement déterminé à renforcer les capacités statistiques nationales par une approche inclusive fondée sur la mobilisation de l'implication nationale, le soutien des donateurs et les partenariats régionaux et nationaux.

L'approche de l'ISU est régie par les besoins et demandes des pays disposant d'une définition précise de leurs priorités politiques, qui doivent trouver un écho dans le Cadre d'indicateurs national (NIF) en éducation. Cet outil sert à déterminer les besoins en données pour la surveillance d'un point de vue national et doit être le point de départ de tout plan visant à améliorer le système d'information sur l'éducation et la collecte des données. Aligner le NIF avec les cadres d'indicateurs internationaux peut rationaliser la collecte des données, permettre de satisfaire aux définitions et normes dans le secteur de l'éducation et d'intégrer les ODD dans les processus de planification nationale.

La Figure 8 présente un aperçu du modèle de I'ISU en matière de soutien au développement des capacités. Cette figure montre les différentes étapes de ce processus séquentiel, qui s'appuie sur le soutien apporté par l'ISU aux systèmes statistiques nationaux pour de multiples objectifs, notamment la cartographie des parties prenantes

\section{Figure 8. Modèle de développement des capacités}

Formulation du

Cadre

d'indicateurs

national (NFI)
Cartographie

des données
Cadre d'évaluation

de la qualité des

données (DQAF)
Élaboration

de la SNDSE 
et des sources de données, l'évaluation de la qualité des données, et la définition d'une stratégie nationale et d'un plan d'amélioration dans le domaine des données. Dès le départ, le rôle de l'ISU est équilibré entre la création de connaissances et le soutien aux parties prenantes, partenaires du développement et décideurs, deux éléments garants de la prise en main par les États de leurs systèmes statistiques et de la durabilité de ceux-ci.

Les activités de développement de l'ISU seront guidées par trois principes :

- Développement de partenariats. L'approche de I'ISU pour la surveillance des ODD exige de solides partenariats entre les différents acteurs aux niveaux mondial, régional et national assurant la durabilité des efforts déployés en matière de développement des capacités. Les partenariats régionaux et internationaux peuvent contribuer à éviter les doublons - par exemple lors du soutien de différents partenaires - et à rendre leur aide et leur implication plus efficaces.

- Prise en main nationale. La prise en main par les autorités nationales est l'élément clé de toute stratégie de développement des capacités efficaces et exige que les parties prenantes nationales définissent les priorités du pays.

- Approche holistique. Le développement des capacités statistiques doit éviter les efforts fragmentés et envisager de développer les capacités d'une façon holistique, des capacités institutionnelles au développement des capacités organisationnelles et individuelles.

Le modèle commence par la mobilisation de l'engagement national et du soutien des donateurs, se poursuit par l'évaluation des besoins de capacité et s'achève par l'élaboration d'un plan d'action chiffré qui sera mis en œuvre par les pays en collaboration avec les partenaires régionaux et nationaux (voir l'Annexe 3).

La CSNU a reconnu la nécessité de renforcer les capacités statistiques afin de surveiller les ODD et a créé le Groupe de haut niveau pour le partenariat, la coordination et le renforcement des capacités pour le suivi du programme de développement à l'horizon 2030 (HLG-PCCB). Ses membres comprennent des statisticiens en chef de 23 instituts de statistique nationaux, qui représentent des pays de leurs régions respectives. Le mandat du HLG-PCCB comprend la production récente du Plan d'action mondial du Cap pour les données sur le développement durable, qui présente les actions et normes nécessaires pour procéder à un renforcement des capacités statistiques approprié et réaliser le suivi des ODD. Le plan repose sur six domaines stratégiques, focalisés sur différents sujets tels que la coordination et la gouvernance des instituts de statistique nationaux, la modernisation, l'harmonisation des données, la diffusion, une approche holistique de l'élaboration des données et la mobilisation des ressources pour permettre l'exécution du plan (Keijzer et Klingebiel, 2017). II recommande l'élaboration de « plans d'action visant à améliorer la disponibilité et la qualité des données et indicateurs sectoriels " afin de surveiller la mise en œuvre des ODD (HLG-PCCB, 2017).

Dans ce contexte, I'ISU a également discuté dans le but d'engager une collaboration avec le Partenariat en statistique pour le développement au $21^{\circ}$ siècle (PARIS21) concernant l'articulation des Stratégies nationales de développement des statistiques(SNDS) et des stratégies statistiques sectorielles. Ces discussions ont servi de base à l'élaboration d'un nouveau projet réalisé pour concevoir et mettre en œuvre des initiatives de suivi pilotes de l'ODD 4 dans des pays à faible ou moyen niveau de revenu d'Asie et d'Afrique dans le cadre du Programme de renforcement des capacités en faveur de l'éducation de I'UNESCO (CapED). La première collaboration concrète est imminente et concernera l'un des dix pays pilotes du CapED (Montoya et Naidoo, 2017; PARIS21, 2017).

Le reste de cette section, ci-après, s'articule autour des principales actions de l'ISU, décrites dans la Figure 8, pour soutenir et aider les pays à définir des normes et à élaborer des cadres de suivi de grande qualité, spécifiques à l'Éducation 2030ODD 4. Elles sont présentées dans trois sections principales, à savoir la mise en œuvre du SSNE, la cartographie des sources de données et la disponibilité des informations et l'évaluation de la qualité des sources d'informations sur l'ODD 4. 


\section{CdP Code de pratiques}

DQAF Cadre d'évaluation de la qualité des données

SSNE Système statistique national de l'éducation

NIF Cadre d'indicateurs national

SNDSE Stratégie nationale de développement des statistiques de l'éducation

SNDS Stratégies nationales de développement des statistiques

\subsection{Concevoir et mettre en œuvre une Stratégie nationale de l'élaboration des statistiques de l'éducation}

L'Agenda 2030 et le Cadre d'action Éducation 2030 appellent expliCITÉment l'ISU à jouer un rôle central en matière de fourniture d'expertise technique pour construire et renforcer les systèmes de données nationaux, en particulier dans les pays à faible ou moyen niveau de revenu (UNESCO, 2015, para. 98).

La coordination entre les différentes sources de données (principalement les données administratives, les enquêtes auprès des ménages et les évaluations des apprentissages) au sein d'un pays demeure l'un des principaux défis de la production de données de qualité pour le suivi de l'ODD 4. Généralement, les différentes entités qui créent chaque source de données ne travaillent pas de façon coordonnée. Dans de nombreux pays, les méthodologies de collecte des données et les données brutes ne sont pas toujours partagées entre les entités, si bien qu'effectivement, les données ne peuvent être associées entre les écoles, ménages ou personnes, par exemple. L'existence de « silos » de données crée des lacunes analytiques empêchant d'appréhender les progrès réalisés dans l'éducation. Il existe également des problèmes tenant aux données partielles, qui ne permettent pas de brosser le tableau complet d'un secteur ou d'un sous-secteur. Par ailleurs, les processus de collecte des données dans les pays développés et dans les pays en développement ne sont pas toujours harmonisés et peuvent donc être à l'origine de confusions.

Compte tenu des exigences complexes liées à l'élaboration des statistiques et au suivi de l'ODD 4, il existe un besoin urgent d'approche coordonnée impliquant tous les fournisseurs de données, les principaux utilisateurs de données ainsi que les partenaires nationaux, régionaux et internationaux. Les parties prenantes du SSNE, qui ont pour mission de produire des statistiques officielles utilisées à des fins d'élaboration de politiques et de planification, doivent être en mesure de produire et de partager des statistiques pertinentes, de qualité, recueillies auprès de multiples sources par le biais d'un effort national coordonné ayant pour objet d'améliorer les mécanismes et les processus de la production de données. Dans la pratique, cependant, le SSNE est

\section{Figure 9. Organisation des Stratégies nationales de développement des statistiques (SNDS)}

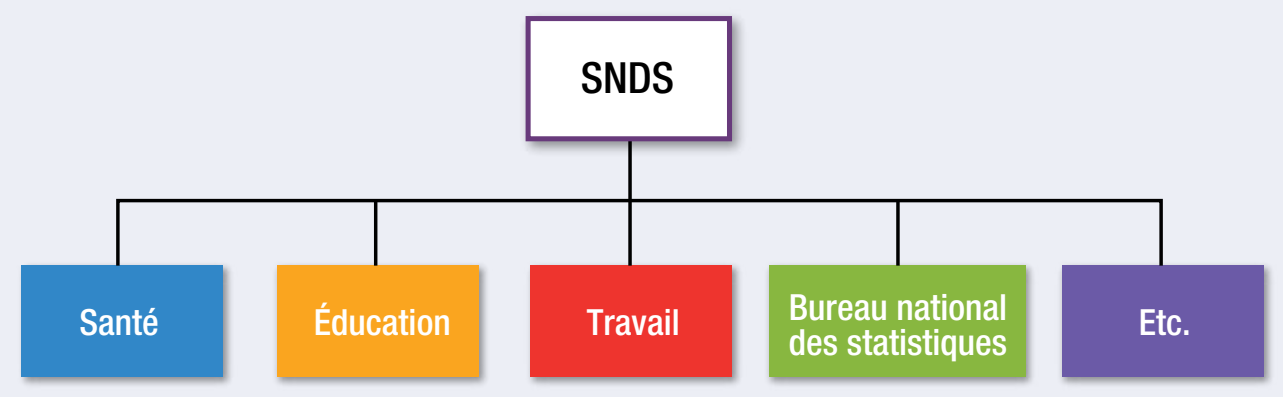


composé de systèmes d'information distincts, qui fonctionnent de façon isolée, et produisent des données dans chaque sous-secteur (par exemple, la petite enfance, l'éducation de base, l'enseignement et la formation techniques et professionnels, l'enseignement supérieur) (voir Section 2.2).

L'ISU soutient le développement d'une approche sectorielle des statistiques de l'éducation qui renforcerait la capacité statistique des SSNE à produire des données permettant de surveiller I'ODD 4. La SNDSE est un instrument politique puissant qui a été conçu par des gouvernements et leurs partenaires ; elle constitue une feuille de route en vue d'un développement du SSNE s'appuyant sur une stratégie holistique des données sur l'éducation. La SNDSE a pour objet d'offrir une vision à moyen terme de l'état d'avancement dans lequel devrait se trouver le SSNE d'ici cinq à dix ans, définit les étapes pour y parvenir et les intègre complètement dans les SNDS. La Figure 9 montre cette relation, la SNDSE étant l'une des stratégies sectorielles du développement des statistiques sur la base de laquelle pourraient se développer les SNDS multisectorielles (HLG-PCCB, 2017 ; PARIS21, 2017).

L'élaboration de la SNDSE est un exercice participatif qui renforce les synergies entre l'ensemble des producteurs et utilisateurs de données sur l'éducation. Le CdP et le DQAF (voir Section 2) sont des éléments centraux du processus d'élaboration de la SNDES ; ils servent d'outils de diagnostic pour évaluer comment la qualité est garantie et comment les performances en termes de qualité peuvent être surveillées grâce au SSNE. L'élaboration de la SNDSE doit satisfaire à plusieurs critères. Elle doit :

- être intégrée dans le processus de préparation, tel que l'exige la NSDS, si elle existe ;

- s'assurer que l'élaboration des statistiques sur l'éducation est entièrement intégrée dans la planification nationale du développement des capacités statistiques ;

- faire écho aux besoins en données sur l'éducation résultant des priorités politiques et de leurs objectifs, y compris les engagements régionaux et internationaux ;

- servir de cadre à l'aide internationale et bilatérale ;

- permettre un suivi approprié et veiller à ce que le processus d'évaluation ait lieu ;
- inclure tous les aspects et unités de la chaîne de production des données, rapprocher les utilisateurs et les producteurs de données, et traiter les questions liées à l'analyse et à l'utilisation des données ; et

- être incluse dans le Plan global de développement sectoriel de l'éducation.

En utilisant une approche sectorielle de l'éducation, la SNDSE doit inclure :

- les activités de production statistique nécessaires pour produire les données requises pour le suivi des objectifs nationaux, régionaux et mondiaux ; et

- les activités de renforcement des capacités visant à s'assurer que les données sont produites conformément aux normes de qualité reconnues.

La SNDSE repose sur une solide analyse, conduite au niveau du pays, de la situation actuelle du SSNE, initialement en termes de disponibilité des données, puis par l'évaluation de la qualité des données des différentes sources et la prise en considération des suggestions d'amélioration. Dans la mesure du possible, elle doit être intégrée dans les plans nationaux relatifs à l'éducation (voir Encadré 10).

La SNDSE est élaborée et mise en œuvre par l'intermédiaire du Groupe d'experts nationaux des données de l'éducation (NEGED), qui offre un espace de dialogue entre le gouvernement et les partenaires pour améliorer le soutien (Montoya et Naidoo, 2017). La structure de la SNDSE s'inscrit dans un cadre axé sur les résultats (y compris les plans chiffrés qui leur sont associés) et devrait être liée aux documents de planification nationaux. Dans de nombreux pays, cependant, la coordination et l'intégration du secteur de l'éducation dans la SNDSE sont faibles et inefficaces, et ont besoin d'un environnement technique et institutionnel approprié. La participation des partenaires du développement au NEGED, grâce auquel des synergies pourraient être créées, est un axe potentiel de l'aide internationale et bilatérale.

Avant qu'un pays ne se lance dans la collecte de données, il doit déterminer quelles sont les exigences en matière de données pour produire chacun des indicateurs de l'ODD 4 (voir Section 2.2 et Section 3.2 sur les métadonnées). La cartographie minutieuse des besoins en données, la disponibilité des données et les évaluations de la qualité des sources de données sont donc des éléments fondamentaux de la conception d'une stratégie réussie et en mesure de renforcer le SSNE, y compris la conception/développement d'un programme de renforcement des capacités. 
Les plans et politiques nationaux d'éducation indiquent souvent quels sont les objectifs du pays pour résoudre les problèmes auxquels est confronté le système éducatif. Un suivi régulier des progrès accomplis depuis le début de la période de planification devrait constituer l'un des aspects de la mise en œuvre de ces plans et politiques. Un cadre de suivi comportant un ensemble d'indicateurs peut fournir les informations de qualité nécessaires pour répondre à ces besoins analytiques.

L'ensemble d'indicateurs utilisé pour surveiller les objectifs en matière d'éducation doit être lié aux priorités politiques définies par les gouvernements. Étant donné que les pays ont généralement un large éventail de priorités nationales en matière d'éducation, et qu'ils ont adopté l'ODD 4 et, possiblement, d'autres programmes de développement supranationaux, ils sont confrontés au défi considérable de produire différents indicateurs et données destinés à répondre à différents niveaux de suivi. Par conséquent, les pays doivent améliorer l'alignement des demandes de données et renforcer les synergies entre l'ensemble des producteurs et des utilisateurs de données. Ces efforts amélioreront à leur tour la qualité des données de l'éducation en tant qu'outils de la définition des politiques et de leur suivi, tout en réduisant la charge de la communication des données.

\subsection{Cartographier les sources de données et la disponibilité des informations}

La cartographie des sources de données et de la disponibilité des informations permet de disposer d'éléments pertinents pour analyser et comprendre les capacités actuelles d'un pays à créer des indicateurs de l'ODD 4. L'exercice de cartographie des données doit reposer sur le Cadre d'indicateurs national (NIF), qui découle des priorités politiques nationales, et inclure les demandes de données qu'impliquent les indicateurs mondiaux et thématiques, à condition qu'elles soient reconnues par le pays et pertinentes.

Une fois que les exigences en matière de données ont été définies par le NIF, les pays doivent être soumis à un processus analytique qui examine si le pays réalise les enquêtes nécessaires pour fournir les données. Les données de qualité doivent correspondre au concept, aux définitions et aux méthodologies de l'indicateur et se conformer à d'autres caractéristiques, telles que la fréquence des données et leur capacité à être ventilées par âge, sexe, lieu, revenu, ethnicité, statut migratoire et handicap afin de pouvoir répondre de façon appropriée au principe fondamental de l'ODD consistant à « ne laisser personne à la traîne » (SGONU, 2016). Les lacunes des données sont particulièrement préoccupantes en termes de qualité des indicateurs de l'ODD 4. Comme indiqué précédemment, l'ISU a calculé qu'environ la moitié seulement des données nécessaires pour suivre les progrès réalisés sur la voie des cibles de l'ODD 4 sont collectées dans le monde (voir Section 1.3) ${ }^{17}$.

Le Questionnaire de cartographie des données de l'ODD 4 a été spécifiquement conçu pour évaluer la disponibilité des données et recueillir les informations nécessaires pour produire les indicateurs proposés en vue du suivi des objectifs du Cadre d'action Éducation 2030ODD 4. Les données sont utilisées pour produire un Recensement détaillé de la disponibilité des données nécessaires pour surveiller l'ODD4 (ISU, 2017a). Cet inventaire initial de la disponibilité des données du pays est la base utilisée pour engager un processus de planification visant à renforcer les systèmes d'information nationaux liés à l'ODD 4. Les informations collectées dans l'inventaire permettent d'identifier les lacunes potentielles dans les données et les domaines qui nécessitent des

\footnotetext{
${ }^{17}$ Des rapports régionaux sur la disponibilité des données ont été publiés pour les États arabes, l'Asie et le Pacifique, l'Amérique latine et les Caraïbes ainsi que l'Afrique subsaharienne, et sont disponibles à l'adresse suivante : http://uis.unesco.org/en/topic/sustainable-development-goal-4
} 
travaux plus approfondis. Par ailleurs, elles étayent l'élaboration d'un plan d'action visant à améliorer la qualité des données et à développer des capacités nationales afin d'aligner et de renforcer les données nationales et le SIGE.

L'outil élaboré par I'ISU pour inventorier la disponibilité des données normalise l'identification des éléments et sources de données pertinentes pour les indicateurs de l'ODD 4 et inclut un Modèle de rapport sur les résultats (voir Tableau 2). Les aspects couverts par le Questionnaire de cartographie des données de l'ODD 4 sont les suivants.

- La disponibilité des données et les possibilités de ventilation par indicateur de I'ODD 4.

- Une brève caractérisation de chacune des sources statistiques nationales pertinentes.

- L'identification des sources possibles auprès desquelles les données manquantes requises pourraient être collectées.

- La participation aux enquêtes régionales et internationales.
Ensemble, ces éléments recensent les sources de données existantes dont la qualité devrait être évaluée ultérieurement grâce à des outils normalisés créés autour d'un cadre qui intègre les principes de qualité essentiels de l'ISU présentés à la Section 2.1. Les principales sources de données requises pour alimenter les indicateurs du suivi de l'ODD 4 - c'est-à-dire les données administratives, les données financières, les enquêtes auprès des ménages et les résultats d'apprentissage - seront évaluées dans le but d'identifier les actions qui pourraient être menées pour améliorer la situation actuelle.

\section{3 Évaluer la qualité des sources d'information de l'ODD 4}

L'ISU a examiné la structure et les modalités de mise en œuvre du DQAF, dérivé de l'adaptation initiale de l'IMF-DQAF existant, l'outil pour les données de l'éducation. L'ISU a largement utilisé l'Ed-DQAF pour une série d'évaluations diagnostiques des systèmes statistiques nationaux de l'éducation en Asie, en Amérique latine et aux Caraïbes et en Afrique

Tableau 2. Extrait du Modèle de rapport sur les résultats du Questionnaire de cartographie des données de l'ODD 4

\begin{tabular}{|c|c|c|c|c|c|c|c|c|}
\hline Cible & Indicateur & Disponibilité & $\begin{array}{l}\text { Dernières } \\
\text { données } \\
\text { disponibles }\end{array}$ & Par âge & Par sexe & $\begin{array}{c}\text { Par } \\
\text { emplace- } \\
\text { ment }\end{array}$ & $\begin{array}{l}\text { Par la } \\
\text { richesse }\end{array}$ & $\begin{array}{c}\text { Par } \\
\text { invalidité }\end{array}$ \\
\hline \multirow[t]{9}{*}{4.1} & 4.1.1. & Oui & 2014 & $x$ & $X$ & $X$ & & \\
\hline & 4.1.2. & Partiel & 2015 & & & & & \\
\hline & 4.1.3. & Oui & 2015 & & $X$ & & & \\
\hline & 4.1.4. & Oui & 2015 & $X$ & $X$ & $x$ & & \\
\hline & 4.1.5. & Non & & & & & & \\
\hline & 4.1.6. & Oui & Pas registré & & $X$ & $x$ & & \\
\hline & 4.1.7. & Oui & 2013 & & & & & \\
\hline & 4.1.x. & & & & & & & \\
\hline & - & & & & & & & \\
\hline
\end{tabular}

Source : Institut de statistique de I'UNESCO, 2017. 
subsaharienne. Il est considéré comme l'un des principaux piliers des activités de l'ISU en matière de renforcement des capacités nationales.

Les Ed-DQAF ont été mis en œuvre en Amérique latine et en Afrique subsaharienne entre 2005 et 2006. L'ISU, en collaboration avec le bureau de I'UNESCO à Dakar (ancien BREDA), a effectué au total 19 évaluations en Afrique subsaharienne avec quelques modifications de la méthodologie - entre 2008 et 2011 - et plus récemment au Cameroun et au Bénin, respectivement en 2012 et 2013. Ces évaluations ont apporté un soutien national et régional au Plan d'action de la seconde Décennie de l'éducation pour l'Afrique de l'Union africaine (2006-2015), au programme pour l'éducation de la Communauté de développement d'Afrique australe et à la Communauté économique des États d'Afrique centrale. Entre 2008 et 2011, l'EdDQAF (la matrice originale et une version révisée) a été appliqué dans 15 pays de la Communauté de développement d'Afrique australe (SADC) et les recommandations ont été largement appliquées par les gouvernements pour améliorer leur SIGE. Dans le cadre d'un projet financé par le ministère australien des Affaires étrangères et du commerce (DFAT), I'Ed-DQAF a été utilisé dans six pays de la région Pacifique en vue de l'élaboration de plans d'action et d'amélioration et pour mobiliser les ressources. Afin d'élargir l'utilisation nationale de l'outil à l'autoévaluation du SIGE, un DQAF en ligne a été élaboré pour 11 autres pays de la région et utilisé pour identifier des solutions communes répondant aux principales interrogations en matière de qualité (ISU, 2017g).

L'ISU promeut l'adoption du CdP afin d'orienter le développement de données de meilleure qualité en mettant en œuvre des Ed-DQAF spécifiques aux sources. Chaque outil ciblé évalue des données administratives, des données d'enquêtes auprès des ménages, des données sur les dépenses publiques d'éducation et des données sur les résultats d'apprentissage : les résultats des évaluations enrichiront les stratégies de soutien mises en œuvre par l'ISU auprès des SSNE.

\subsubsection{Analyse de la qualité des données de systèmes administratifs courants}

Les dossiers administratifs constituent la source de 27 indicateurs de suivi de l'ODD 4 et fournissent des informations sur les élèves, les enseignants et les établissements (voir Tableau 3). Ces données sont collectées au niveau de l'école, puis communiquées via la chaîne administrative au ministère ou à l'institut de statistique national.

Les données sont utilisées par les ministères de l'éducation pour la gestion, à des fins de planification et/ou statistiques, et sont généralement mises à jour régulièrement. La plupart des indicateurs de suivi internationaux élaborés pour l'agenda de développement mondial précédent reposaient sur des données administratives produites par des pays et compilées par des organisations internationales.

Dans le contexte de l'ODD 4-Cadre d'action Éducation 2030 et avec la nécessité, pour les pays, de renforcer leur SSNE, l'Ed-DQAF avait besoin d'une refonte complète pour être en mesure de relever les nouveaux défis. Plus précisément, l'Ed-DQAF avait besoin d'être révisé en termes de contenu, de structure et de modalités de mise en œuvre. L'Ed-DQAF original était légèrement contraignant en termes d'utilisation et certains concepts avaient besoin d'être ajustés. Sur la base de l'examen, I'ISU a produit un nouvel Ed-DQAF allégé en mai 2017, avec une matrice réduite qui conserve néanmoins la plupart des dimensions initiales du cadre de la qualité (voir Encadré 11). Les équipes et les experts statistiques nationaux peuvent utiliser la version allégée de l'Ed-DQAF comme un outil d'autoévaluation suivant les normes reconnues pour tous les types de sources de

\section{Tableau 3. Indicateurs de l'ODD 4 par source de données}

\begin{tabular}{|c|c|c|c|c|}
\cline { 2 - 5 } & Administrative & $\begin{array}{c}\text { Dépenses publiques } \\
\text { d'éducation }\end{array}$ & $\begin{array}{c}\text { Enquête auprès des } \\
\text { ménages }\end{array}$ & $\begin{array}{c}\text { Évaluation des résultats } \\
\text { d'apprentissage }\end{array}$ \\
\hline $\begin{array}{c}\text { Nombre total de cibles de } \\
\text { l'ODD 4 }\end{array}$ & 27 & 2 & 19 & 6 \\
\hline
\end{tabular}

Note : Voir l'Annexe 4 pour des informations détaillées par cible de l'ODD 4. 
En 2004, I'ISU a entrepris des travaux préparatoires, avec un groupe d'experts de la Banque mondiale, afin d'adapter spécifiquement l'outil DQAF3 du FMI aux données sur l'éducation. L'Ed-DQAF - une matrice de 140 éléments qualité structurés autour des six dimensions de la qualité - a été utilisé pour une série d'exercices d'évaluation nationaux dans des pays en développement. Pour faire de cet outil un bien public, un portail de documentation Wiki en ligne a été créé pour centraliser toute la documentation existante et donner accès à tous les rapports sur l'Ed-DQAF et à tous les documents connexes.

Dans le contexte de l'ODD 4-Cadre d'action Éducation 2030 et de la révision de la stratégie de renforcement des capacités de l'ISU, les équipes statistiques nationales demandaient un outil d'autoévaluation plus léger mais conforme aux normes reconnues. L'Ed-DQAF a donc été spécifiquement focalisé sur les systèmes courants de données administratives de l'éducation (alors que d'autres outils ont été élaborés pour les données sur l'éducation collectées par le biais des enquêtes auprès des ménages et pour les évaluations des apprentissages). Par ailleurs, il a été focalisé sur les critères de l'autoévaluation de manière à ce que les éléments probants de l'évaluation puissent être identifiés de façon réaliste par les équipes nationales, pour chacun des éléments de la qualité des données sélectionnés. Le nouvel Ed-DQAF allégé conserve 46 des 140 éléments de la matrice initiale. La nouvelle matrice comprend des orientations pratiques, en matière de notation, pour chaque rubrique de la qualité, ce qui facilite l'autoévaluation effectuée par les équipes nationales. Elle fournit des éléments d'orientation aux structures chargées de produire des données fiables et appropriées, qui respectent les normes professionnelles et scientifiques reconnues au plan international.

Le CdP a également été adapté en une version plus courte et opérationnalisé conformément à la version allégée de l'Ed-DQAF. À l'instar de la version complète du CdP qui comprend 12 principes (voir l'Encadré 6), la version allégée comporte huit principes et 21 indicateurs couvrant le contexte institutionnel, les processus de production statistique et les résultats statistiques : le cadre politique et juridique, l'adéquation des ressources, la pertinence, la solidité de la méthodologie, l'exactitude et la fiabilité, la périodicité et la ponctualité, et l'accessibilité et la clarté (voir l'Annexe 1).

Source : Institut de statistique de l'UNESCO, 2012b, 2017b.

données (par exemple les dossiers administratifs sur l'éducation, les recensements dans les écoles, les enquêtes auprès des ménages, les évaluations des résultats d'apprentissage, les données sur les finances de l'éducation).

\subsubsection{Analyse de la qualité des données pour les sources des données relatives aux dépenses publiques pour l'éducation}

Les données relatives aux dépenses comprennent des informations sur les dépenses publiques consacrées à l'éducation extraites de rapports sur l'exécution budgétaire, par exemple sur la construction et l'entretien des écoles ou les salaires des enseignants. Concernant les données relatives au financement de l'éducation, I'ISU a conçu trois outils pour aider les pays à inventorier les flux de financement de l'éducation, dresser la liste des données disponibles pour surveiller le financement de l'éducation et évaluer la qualité des données relatives au financement de l'éducation. Ces trois outils complémentaires, conçus pour être utilisés dans la séquence suivante, sont dénommés Inventaire des flux de financement de l'éducation, Matrice des sources de données et Cadre d'évaluation de la qualité des données : Sources des données relatives aux dépenses publiques consacrées à l'éducation (ci-après, DQAF Outil financement) ${ }^{18}$.

${ }^{18}$ Le DQAF-Outil financement est dérivé de l'Ed-DQAF pour les données administratives. 
En juin 2017, l'ISU a élaboré le DQAF Outil financement pour aider les responsables de l'éducation à évaluer d'un œil critique la qualité des données produites par leurs collègues du ministère des Finances, et faire en sorte qu'ils s'en servent pour dialoguer avec eux. L'objectif du DQAF Outil financement est d'utiliser l'outil principal du DQAF, conçu pour les données administratives, et de l'adapter pour évaluer la qualité des données relatives au financement de l'éducation provenant de sources gouvernementales. Relativement courte, la matrice de 12 questions s'articule autour des trois mêmes domaines principaux - contexte institutionnel, processus de production statistique et résultats statistiques - et se focalise sur la façon dont les données sur les finances publiques peuvent être utilisées par les parties prenantes du secteur de l'éducation (voir l'Annexe 5). En tant que tel, il n'a pas pour objectif d'évaluer la qualité globale des systèmes financiers gouvernementaux.

L'outil évalue avec quelle rapidité et quelle facilité les données relatives au financement sont mises à la disposition des statisticiens et décideurs de l'éducation et détermine si elles peuvent être ventilées d'une façon qui est pertinente pour le secteur de l'éducation, notamment par niveau d'éducation ou par catégorie de dépenses (par exemple, livres scolaires). Il est utilisé conjointement avec les outils Inventaire des flux de financement de l'éducation et Matrice des sources de données créés par l'ISU, qui servent à déterminer les sources et les modalités de financement de l'éducation d'un pays et à relever les sources de données correspondantes. Les résultats combinés des trois évaluations doivent inclure les recommandations et actions correspondantes et résoudre les problèmes en suspens, et doivent être inclus pour prise en considération dans les SNDSE.

\subsubsection{Analyse de la qualité des données des enquêtes auprès des ménages}

Les enquêtes auprès des ménages sont la source de 19 indicateurs de suivi de l'ODD 4 (voir Tableau 3). En dépit de leur diversité, les enquêtes auprès des ménages fournissent généralement des informations sur l'éducation du point de vue de la demande, telles que la participation et l'achèvement (ou la non-participation) de la population, le niveau d'instruction parmi les adultes et les taux d'alphabétisation. Les vastes enquêtes auprès des ménages, telles que l'Enquête démographique et de santé (DHS) et l'Enquête par grappes à indicateurs multiples (MICS) de I'UNICEF, sont extrêmement utiles pour produire des statistiques sur les occasions d'apprentissage offertes aux enfants avant leur entrée au primaire ${ }^{19}$. Compte tenu de la richesse des données socioéconomiques et des autres informations sur les ménages qu'elles contiennent, ces deux enquêtes sont particulièrement précieuses pour produire des statistiques essentielles sur l'éducation. Certains programmes nationaux et internationaux d'enquêtes auprès des ménages, notamment le cycle actuel de la MICS, collectent également des données sur les compétences de base en lecture et en mathématiques. Certaines enquêtes auprès des ménages sont focalisées sur l'éducation, comme les Enquêtes auprès des ménages DHS EdData.

Plus courantes, cependant, sont les enquêtes auprès des ménages couvrant plusieurs sujets, l'éducation étant dans ce cas un sujet d'intérêt parmi plusieurs autres. Les données sur l'éducation des enquêtes auprès des ménages sont utilisées pour compléter les données administratives nationales, en particulier en ce qui concerne la ventilation des données pour les différents groupes, y compris les enfants non scolarisés ${ }^{20}$. Les données des enquêtes auprès des ménages sont largement utilisées dans les documents nationaux de planification de l'éducation ainsi que dans les comparaisons infranationales et internationales établies par I'UNICEF, I'ISU, la Banque mondiale et d'autres fournisseurs et consommateurs de statistiques de l'éducation.

Pour les utilisations en rapport avec l'éducation, il est important d'évaluer la qualité et la durabilité des données des enquêtes auprès des ménages portant sur l'éducation. Les principales questions auxquelles il convient de répondre sont, par exemple : est-ce que les données appropriées sont collectées ? Est-ce qu'elles sont suffisamment fiables ? Est-ce qu'elles sont représentatives de la population?

\footnotetext{
${ }^{19}$ Le programme DHS est mis en œuvre par ICF International et financé par l'Agence des États-Unis pour le développement international (USAID).

${ }^{20}$ Les données des enquêtes auprès des ménages sont généralement ventilées par sexe, âge, lieu et richesse, quelquefois par ethnicité et langue maternelle, et rarement en termes de handicap (l'actuelle Série 6 de la MICS ajoute cette catégorie).
} 
L'ISU a conçu un outil pour évaluer les données des enquêtes auprès des ménages sur l'éducation. II s'inspire largement du DQAF conçu pour les données administratives et suit les huit principes du CdP décrits dans l'Encadré 11. L'outil du DQAF pour les données des enquêtes auprès des ménages (DQAF-HHS), qui aide les pays à évaluer l'utilité des différentes sources de données des enquêtes auprès des ménages pour les statistiques sur l'éducation, comprend deux tableurs. Le premier tableur collecte les informations sur le contexte institutionnel et les ressources, et est commun à toutes les enquêtes réalisées dans le pays. Le second collecte les informations de base spécifiques à l'enquête auprès des ménages et inclut des informations sur les processus statistiques et les résultats statistiques. En tant que tel, le DQAF-HHS partage avec l'Ed-DQAF certains aspects sur les données administratives, mais est davantage focalisé sur les aspects spécifiques aux enquêtes liés à la collecte, à l'analyse et à la diffusion des données. Ceci s'applique en particulier au Principe 8 (Exactitude et fiabilité), qui encadre l'échantillonnage, la couverture en termes de population et les options en matière de ventilation.

\subsubsection{Analyse de la qualité des données des évaluations des résultats d'apprentissage}

L'une des composantes essentielles du Cadre d'action Éducation 2030-ODD 4 est que les enfants et les adultes atteignent un niveau minimum de connaissances et de compétences dans certains domaines d'apprentissage, à savoir la lecture (ou la littératie), la numératie, le développement (pour la petite enfance), les compétences techniques et professionnelles, les sciences de l'environnement et de la Terre (pour les adolescents de 15 ans) ainsi que les connaissances et compétences visant à promouvoir le développement durable. Au total, six indicateurs sont susceptibles de ressortir des évaluations des résultats d'apprentissage tels que définis par les Cibles 4.1, 4.2, 4.4, 4.6 et 4.721. Faire état des progrès réalisés sur la voie de ces cibles exige de produire des indicateurs qui mesurent ces domaines : les évaluations à grande échelle sont des instruments importants pour établir et surveiller la qualité de l'éducation au niveau du système.
Les évaluations à grande échelle se focalisent sur des domaines bien définis (par exemple la lecture ou les mathématiques), généralement mesurés à l'aune des apprentissages requis par le programme national aux différents niveaux et définis dans le cadre d'évaluation. Elles ont pour objectif de répondre à la question de savoir si l'apprenant peut appliquer les compétences et concepts acquis en classe. Les évaluations des apprentissages peuvent être à portée internationale, régionale ou nationale, se focaliser sur une population particulière (par exemple, par âge ou par classe) et peuvent reposer sur des échantillons ou être conduites comme un recensement.

Pour être efficaces, les évaluations à grande échelle doivent rassembler des données qui reflètent exactement la situation présente. En tant que telle, la gestion de la qualité des données joue un rôle central dans l'ODD 4. Au niveau national, cela implique l'élaboration de stratégies nationales pour les évaluations à grande échelle, les données sur l'éducation et l'engagement de renforcer les capacités en matière d'évaluation et de statistiques. Au niveau international, cela implique une approche participative de l'élaboration des normes et méthodologies internationales, la fourniture d'outils de diagnostic et de principes directeurs, et le soutien du développement des capacités (ISU, 2016).

En conséquence, l'ISU propose un cadre visant à guider la conception et la mise en œuvre des évaluations de l'apprentissage qui contribuera à une production des mesures des résultats d'apprentissage qui soit conforme aux normes techniques de grande qualité : les Principes des bonnes pratiques dans l'évaluation des apprentissages (GP-LA) constituent un élément central de l'engagement international en matière de gestion de la qualité des données pour les résultats d'apprentissage de l'ODD 4 (ISU et ACER, 2017). Les GP-LA sont une articulation des bonnes pratiques qui peuvent fonctionner avec un large éventail d'activités d'évaluation des apprentissages menées à travers le monde. Dans le cadre des processus de communications des données de l'ODD 4, les GP-LA ont les deux fonctions suivantes.

\footnotetext{
${ }^{21}$ Celles-ci correspondent aux Indicateurs 4.1.1, 4.2.1, 4.4.2, 4.6.1, 4.7.4 et 4.7.5.
} 
- Premièrement, ils servent de cadre conceptuel pour évaluer la qualité des évaluations à grande échelle et des données de ces évaluations soumises pour établir des rapports sur l'ODD 4. En mettant en exergue les principes clés de la qualité des évaluations, les GP-LA aident les pays à atteindre une rigueur technique leur laissant suffisamment de souplesse pour définir leurs propres priorités en matière d'évaluation.

- Deuxièmement, les principes des GPLA, complétés par le code de pratiques correspondant, étayeront le diagnostic des capacités des pays à collecter, à analyser et à utiliser les données des évaluations à grande échelle. Dans les cas où les données des évaluations à grande échelle soumises pour établir des rapports sur l'ODD 4 ne satisfont pas aux normes requises en matière de communication des données, les GP-LA faciliteront la formulation de plans de renforcement des capacités et le ciblage du soutien technique.

Les GP-LA sont une déclaration de principes conçue pour faciliter l'élaboration et la mise en œuvre des programmes d'évaluation. Les déclarations sont délibérément générales de façon à ce qu'elles soient applicables dans différents contextes et environnements d'évaluation à grande échelle (par exemple, les évaluations centrées sur un pays, une région ou le monde; sur les écoles ou les ménages). Ainsi, les GP-LA constituent le cadre conceptuel permettant d'élaborer une évaluation de la qualité des données pour ce type d'étude. S'appuyant sur les déclarations proposées par les GP-LA, I'ISU élabore les outils permettant de mettre en œuvre l'évaluation de la qualité des données des études portant sur les résultats d'apprentissage et sur leur capacité à satisfaire aux exigences des indicateurs d'apprentissage de l'ODD 4. Ce processus sera mis en œuvre en deux étapes. Basé sur le cadre conceptuel proposé par le GP-LA, I'ISU développe une méthodologie connue sous le nom de processes de l'alignement des données pour obtenir des informations internationalement comparables nécessaires pour suivre les résultats de l'apprentissage pour l'Indicateur 4.1.1 des ODD (voir le Tableau 1). Le processus d'alignement des données permettra les systèmes d'éducation à examiner et à signaler sur le niveau actuel d'alignement des programmes d'évaluation avec les Rapports d'échelles de l'ISU (voir Encadré 9) et sera mis en œuvre six étapes (ISU et ACER, à paraître):
1. La sensibilisation du pays à la cohérence internationale dans le compte rendu des résultats d'apprentissage : Le pays se familiarise avec la définition et description des échelles de rapports de I'ISU, la couverture du contenu, les domaines, les brins et les niveaux.

2. Préparation au niveau national des bases de données et des matériaux : Le pays compile son cadre des résultats d'apprentissage national et internationale, test des plans, itèmes, réponses, base de données micro, livres de code, manuels opérationnels et résultats d'évaluation.

3. Alignement conceptuel : cela définit l'alignement conceptuel du programme d'évaluation avec le Rapport d'échelles de I'ISU, par domaine (par exemple, mathématiques, lecture). Dans chaque domaine, l'alignement est vérifié dans les brins et pour les niveaux de progrès dans l'apprentissage pour chaque brin et pour l'ensemble du domaine.

4. Évaluation de la cohérence procédurale : cette étape permet à l'ISU de collecter des informations sur les procédures des systèmes d'éducation sur les données qui sont fournies pour l'Indicateur 4.1.1, et pour comprendre le niveau de confiance avec lequel les résultats peuvent être signalés.

5. Validation et reporting : un processus de validation y compris les composants les plus critiques de l'alignement conceptuel et l'évaluation de cohérence procédurale est mise en œuvre, avec l'aide d'un évaluateur externe, avant soumettre les informations pour un reporting global de l'indicateur.

\section{Plan d'amélioration et renforcement des} capacités :

Le processus d'alignement des données générera déclarations sur les limitations d'alignement avec le Rapport d'échelles de l'ISU. Cette information est destiné à aider un pays à travailler vers une meilleure précision dans les rapports pour l'Indicateur 4.1.1. Le travail de l'ISU à travers le GAML comprend outils et processus supplémentaires pour aider pays dans la planification du renforcement des activités de capacité.

\subsection{Vers une approche coordonnée de la production des données sur l'éducation}

L'ensemble d'outils décrits dans les parties précédentes de la Section 3 a été conçu par l'ISU pour accompagner les systèmes statistiques 
nationaux dans leurs projets visant à améliorer la qualité des données sur l'éducation produites aux niveaux national et international pour surveiller l'ODD 4 (voir Figure 10).

L'ISU travaille d'une façon coordonnée en développant les capacités techniques des équipes statistiques des pays afin qu'elles élaborent un plan pour améliorer le SSNE. Dans ce plan, la formulation du NIF est le point de départ qui déterminera les besoins en données. Puis, en mettant en œuvre le Questionnaire de cartographie des données ainsi que les différents DQAF, les pays peuvent générer la plus grande partie des apports nécessaires pour alimenter le développement d'une SNDSE à des fins d'élaboration de politiques et de planification, et donc intégrer la qualité des données dans un système statistique coordonné plus vaste.L'initiative pilote CapED ODD 4 a été lancée à la fin du premier semestre 2016 dans le cadre du Programme de l'UNESCO CapED. Son objectif était de développer des capacités nationales afin d'intégrer l'ODD 4 dans les politiques nationales d'éducation et dans la gestion du secteur, y compris le suivi des progrès de l'ODD 4.

L'initiative CapED ODD 4 constitue une plateforme qui permet de tester et de mettre en œuvre cette approche et les outils qui lui sont associés dans un groupe de pays à faible ou moyen niveau de revenu sélectionnés. Les dix pays pilotes qui sont aidés par l'UNESCO sont : I'Afghanistan, le Cambodge, la République démocratique du Congo, Haïti, Madagascar, le Mali, le Mozambique, le Myanmar, le Népal et le Sénégal. L'ISU, en tant que membre de l'équipe de mise en œuvre de l'UNESCO sur le projet, a élaboré des matériels de formation sur I'utilisation des outils de suivi des données. L'ISU travaille en partenariat avec les pays dans un processus d'apprentissage par l'expérience basé sur des outils simples qui peuvent être adaptés au contexte national (Montoya et Naidoo, 2017).

\section{Figure 10. Composantes de l'ISU pour soutenir la production et les outils nationaux de données sur l'éducation}

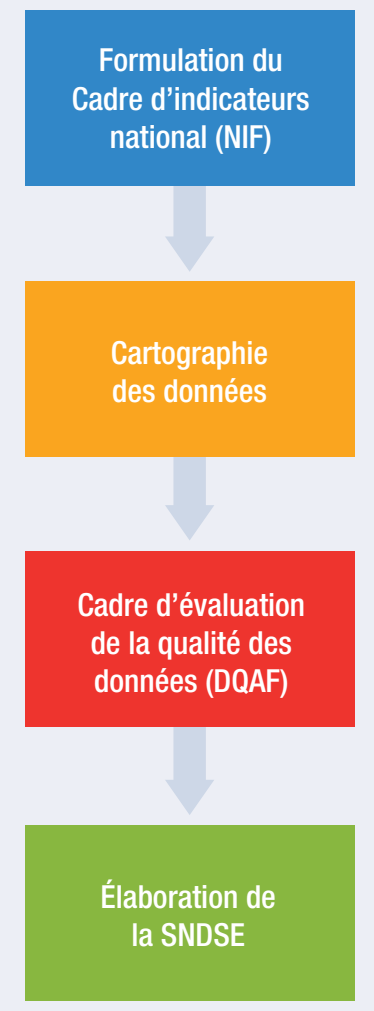

- Principes directeurs de I'ISU/CapED

- Outil NFI

- Document sur les métadonnées

- Questionnaire de cartographie des données de l'ODD 4

- Données administratives

- Données relatives aux dépenses publiques consacrées à l'éducation

- Enquêtes auprès des ménages

- Évaluations des résultats d'apprentissage

- Directives NSDES 


\section{Conclusion}

Au cours de l'année passée, l'ISU et ses partenaires ont continué à conduire la mise en œuvre des indicateurs mondiaux et thématiques pour suivre le Cadre d'action Éducation 2030-ODD 4, comme il en avait été convenu. II a avancé dans l'élaboration de normes et d'outils spécifiques visant à soutenir et à renforcer les systèmes d'information nationaux sur l'éducation pour répondre aux nouvelles exigences en matière de suivi.

Ce rapport place la qualité des données à l'avantplan de la stratégie de mise en œuvre des cadres de suivi. Ainsi, il propose une approche pour définir la qualité des données et une marche à suivre pour élaborer des indicateurs de la qualité dans le secteur de l'éducation aux niveaux national et international.

L'ISU peut apporter une assistance technique aux pays qui leur permettra de développer leurs capacités statistiques nationales et de fournir des données de qualité pour les indicateurs de I'ODD 4. À ces fins, I'ISU pourra coopérer avec les pays à toutes les étapes ou à une étape spécifique du processus de production. L'Initiative pilote du Programme CapED de I'UNESCO met en pratique cette approche en tenant compte des défis complexes du suivi et en fournissant une planification rigoureuse pour améliorer la production et la mise à disposition des indicateurs nécessaires. Cela doit inclure des évaluations de la qualité des sources de données existantes.

Le cadre permettant de guider la production de données de qualité sur l'éducation présenté dans ce rapport est bien aligné avec les cadres de l'assurance qualité des données internationaux, régionaux et, souvent, nationaux. II repose sur la création de codes de pratiques spécifiques aux sources de données pertinentes pour l'éducation qui définissent des principes de qualité pour chacun des trois grands domaines identifiés dans les processus de production statistique : le contexte institutionnel, le processus statistique proprement dit et les résultats statistiques.

Le rapport a relevé certaines actions qui sont fondamentales pour créer les conditions d'un suivi approprié du Cadre d'action Éducation 2030-ODD 4. D'autres travaux sont nécessaires pour accélérer le soutien du développement des méthodologies et normes de production des données pour les indicateurs de l'ODD 4 qui ne font pas encore l'objet de communications de données. Par ailleurs, la couverture mondiale de certains des indicateurs de l'ODD 4 existants est faible et plusieurs pays sont à la traîne en matière d'établissement des rapports et de suivi des progrès sur la voie des objectifs de l'ODD 4. L'un des aspects particulièrement critiques de la communication des données sur les ODD est la mesure de l'équité dans l'ensemble des sources de données utilisées pour les indicateurs sur l'éducation.

Tous ces aspects sont au cœur de la mise en $œ u v r e$ des processus de suivi de l'ODD 4. Ces défis ne peuvent être relevés sans renforcer le rôle, la responsabilité et les ressources des gouvernements dans la production de données de qualité. Les pays et les organisations internationales ont besoin d'une aide financière pour consacrer l'expertise et le temps nécessaires à la construction des fondations de systèmes de suivi nationaux et internationaux en vue de produire des indicateurs efficaces et comparables au plan international.

Mettre en œuvre les processus de suivi de l'ODD 4 exige également une coordination importante entre les entités internationales, régionales et nationales impliquées dans la collecte et la communication des données. Au niveau supranational, il est fondamental d'éviter la reproduction des efforts à l'identique, et donc de multiplier les charges imposées aux capacités nationales en matière de communication des données, et d'envoyer des messages contradictoires depuis les organisations internationales. Au niveau national, une stratégie sectorielle de l'éducation, telle que la SNDSE proposée ici, est cruciale pour articuler les efforts de toutes les parties prenantes statistiques des pays, et d'assurer une affectation économique des ressources.

Le rôle des partenaires financiers dans le succès de l'élaboration de mécanismes de suivi de la qualité ne doit pas être sous-estimé. II est essentiel de mobiliser des ressources nationales et externes suffisantes, par pays et par donateur, pour soutenir les efforts de la communauté statistique. Des investissements ciblés sont nécessaires pour créer les fondements solides et robustes des systèmes statistiques nationaux et internationaux. Aucun pays ne peut être laissé sur le bord de la route dans le suivi des progrès de l'ODD 4. 


\section{Références}

CCS-UNS (2016). Rapport du Comité des statisticiens en chef du système des Nations Unies. Note du Secrétaire général (Commission de statistique Quarante-huitième session, 7-10 mars 2017 No. E/ CN.3/2017/29). New York : Comité des statisticiens en chef du système des Nations Unies (CCS-UNS). https://unstats.un.org/unsd/statcom/48th-session/documents/2017-29-UNSystem-F.pdf

CCS-UNS (2017). Draft UN Statistics Quality Assurance Framework Including a Generic Statistical Quality Assurance Framework for a UN Agency (Commission de statistique Quarante-huitième session, 7-10 mars 2017). New York : Comité des statisticiens en chef du système des Nations Unies (CCS-UNS). https:// unstats.un.org/unsd/statcom/48th-session/documents/BG-4j-QAF-E.pdf

CEPALC (2011a). Code of Good Practice in Statistics for Latin America and the Caribbean (sixth meeting of the Statistical Conference of the Americas of the Economic Commission for Latin America and the Caribbean in Bávaro, Dominican Republic, 16-18 November 2011). Santiago: United Nations Economic Commission for Latin America and the Caribbean (ECLAC). http://www.dane.gov.co/files/noticias/ BuenasPracticas_en.pdf

CEPALC (2011b). Proposal for a Code of Good Practice in Statistics in Latin America and the Caribbean and Implementation Plan for 2012-2013 (sixth meeting of the Statistical Conference of the Americas of the Economic Commission for Latin America and the Caribbean Bávaro, Dominican Republic 16-18 November 2011). Santiago: United Nations Economic Commission for Latin America and the Caribbean (ECLAC). http://repositorio.cepal.org/bitstream/handle/11362/16393/1/S1100683_en.pdf

CNUCED et Équipe spéciale (2016). United Nations Statistics Quality Assurance Framework (UN-SQAF) Including a Generic Statistical Quality Assurance Framework for a UN Agency (No. UNSYSTEM/2017/3). New York : Conférence des Nations unies sur le commerce et le développement. https://unstats.un.org/ unsd/unsystem/Documents-March2017/UNSystem-2017-3-QAF.pdf

Commission de l'Union africaine (2015a). Agenda 2063 : L'Afrique que nous voulons (Document-Cadre). Addis-Abeba : Union africaine. http://www.africa-platform.org/sites/default/files/resources/au_ agenda_2063_french.pdf

Commission de l'Union africaine (2015b). Agenda 2063. L'Afrique que nous voulons : Cadre stratégique commun pour une croissance inclusive et un développement durable. Premier plan décennal de mise en œuvre 2014-2023. Addis-Abeba : Union africaine. Agenda_2063_First_Ten_Year_Implementation_ Plan_2014_2023_FR.pdf

DAESNU (2016). Progress Towards the Sustainable Development Goals. New York : Département des affaires économiques et sociales des Nations Unies (DAESNU). https://unstats.un.org/sdgs/files/report/2016/ secretary-general-sdg-report-2016--FR.pdf

DAESNU (2017). High-Level Political Forum Timeline. https://sustainabledevelopment.un.org/hlpf (Consulté le 7 septembre 2017).DANE (2017). Plan Estadístico Nacional 2017 - 2022 Documento aprobado y expedido por el Consejo Asesor Nacional de estadística, 27 de abril de 2017. Bogotá: Departamento Administrativo Nacional de Estadística (DANE). https://www.dane.gov.co/files/sen/PEN-2017-2022.pdf

DSNU (2012). National Quality Assurance Framework. New York, NY : Division de la statistique des Nations Unies (DSNU). https://unstats.un.org/unsd/dnss/docs-nqaf/GUIDELINES\%208\%20Feb\%202012.pdf (Consulté le 9 septembre 2017).

DSNU (2013). Principles Governing International Statistical Activities. New York : Division de la statistique des Nations Unies (DSNU). https://unstats.un.org/unsd/methods/statorg/Principles_stat_activities/principles_ stat_activities.pdf (Consulté le 7 septembre 2017). 
ESS (2015). Quality Assurance Framework of the European Statistical System (No. Version 1.2). Luxembourg : Union européenne. Système statistique européen (SSE). http://ec.europa.eu/eurostat/ documents/64157/4392716/ESS-QAF-V1-2final.pdf/bbf5970c-1adf-46c8-afc3-58ce177a0646

Eurostat (2011). Code de bonnes pratiques de la statistique européenne pour les services statistiques nationaux et communautaires (Adopté par le Comité sur système statistique européen). Luxembourg : Commission européenne, Direction générale EUROSTAT. http://ec.europa.eu/eurostat/ documents/3859598/5921941/KS-32-11-955-FR.PDF/f1dfd441-391c-4c15-94f2-b34a43697f55

Eurostat (2017). Indicators for Monitoring the Sustainable Development Goals (SDGs) in an EU Context. EU SDG Indicator Set Final version of 28 April 2017 as agreed with Commission Services, which received a favourable opinion by the European Statistical System Committee at its meeting of 17-18 May 2017. Luxembourg : Commission européenne, Direction générale EUROSTAT E: Sectoral and Regional Statistics Unit E-2: Environmental statistics and accounts; sustainable development. http://ec.europa.eu/eurostat/ documents/276524/7736915/EU-SDG-indicator-set-with-cover-note-170531.pdf

FMI (2012). Data Quality Assessment Framework. Washington, DC : Fonds monétaire international. http://dsbb.imf.org/pages/dqrs/DQAF.aspx

Groupe d'experts sur les Cadres nationaux d'assurance de la qualité des données (2012). Glossary. New York: UNSD. https://unstats.un.org/unsd/dnss/docs-nqaf/NQAF\%20GLOSSARY.pdf

GIAE-ODD (2013). Lessons Learned from MDG Monitoring From A Statistical Perspective Report of the Task Team on Lessons Learned from MDG Monitoring of the IAEG-MDG. New York : Groupe inter-agences et d'experts sur les indicateurs des ODD (GIAE-ODD). https://unstats.un.org/unsd/broaderprogress/pdf/ Lesson\%20Learned\%20from\%20MDG\%20Monitoring_2013-03-22\%20(IAEG).pdf

GIAE-ODD (2016). General principles for refining the indicator framework. Draft - 20 September 2016 (Document de base de la $4^{\text {ème }}$ réunion du GIAE-ODD, 20-21 octobre, à Addis-Abeba, Éthiopie). New York : Groupe inter-agences et d'experts sur les indicateurs des ODD (GIAE-ODD). https://unstats.un.org/sdgs/ files/meetings/iaeg-sdgs-meeting-04/General\%20Principles\%20for\%20Refining\%20the\%20Indicator\%20 Framework.pdf

HLG-PCCB (2017). Cape Town Global Action Plan for Sustainable Development Data. Le Cap, Afrique du Sud : Groupe de haut niveau pour le partenariat, la coordination et le renforcement des capacités pour le suivi du programme de développement à l'horizon 2030 (HLG-PCCB). https://unstats.un.org/sdgs/hlg/ Cape-Town-Global-Action-Plan/

Institut de statistique de l'UNESCO (ISU) (2012a). Classification internationale type de l'éducation 2011. Montréal : Institut de statistique de I'UNESCO (ISU).

Institut de statistique de l'UNESCO (ISU) (2012b). Ed-DQAF Wiki. http://dqaf.uis.unesco.org/index. php?title=Main_Page (Consulté le 7 septembre 2017).

Institut de statistique de I'UNESCO (ISU) (2014). Domaines d'études et de formation de la CITÉ 2013 (CITÉ-F) (Manuel accompagnant la Classification internationale type de l'éducation 2011). Montréal : Institut de statistique de l'UNESCO (ISU). http://uis.unesco.org/sites/default/files/documents/isced-fields-ofeducation-and-training-2013-en.pdf

Institut de statistique de l'UNESCO (ISU) (2016). Jeter les bases pour mesurer l'Objectif de développement durable 4 (Rapport sur les données du développement durable). Montréal: Institut de statistique de I'UNESCO (ISU). http://uis.unesco.org/sites/default/files/documents/laying-the-foundation-to-measuresdg4-sustainable-development-data-digest-2016-fr.pdf

Institut de statistique de l'UNESCO (ISU) (2017a). Outils de renforcement des capacités. Montréal : Institut de statistique de l'UNESCO (ISU). http://uis.unesco.org/fr/outils-de-renforcement-des-capaCITÉs (Consulté le 13 décembre 2017). 
Institut de statistique de I'UNESCO (ISU) (2017b). Ed-Data Quality Assessment Framework (Ed-DQAF) to Evaluate Administrative Routine Data Systems: Training Workshop Manual. Montréal : Institut de statistique de I'UNESCO (ISU).

Institut de statistique de l'UNESCO (ISU) (2017c). Centre de données de l'ISU sur l'éducation. http://data. uis.unesco.org/?lang=fr\&SubSessionld=a2d43869-98aa-4575-a36d-385e435df74c\&themetreeid=-200 (Consulté le 13 décembre 2017).

Institut de statistique de l'UNESCO (ISU) (2017d). Light Data Quality Assessment Framework: Government Education Expenditure Data Sources. Montréal : Institut de statistique de l'UNESCO (ISU). http://uis.unesco. org/en/search/site/data\%20quality\%20assessment\%20framework\%20light\%20administrative?f\%5B0\%5D $=$ type $\% 3$ Adocument

Institut de statistique de l'UNESCO (ISU) (2017e). Métadonnées relatives aux indicateurs mondiaux et thématiques pour le suivi et l'examen de l'ODD 4 et de l'Éducation 2030. Montréal : Institut de statistique de I'UNESCO (ISU). http://uis.unesco.org/sites/default/files/documents/metadata-global-thematic-indicatorssdg4-education2030-2017-fr.pdf

Institut de statistique de l'UNESCO (ISU) (2017f). More Than One-Half of Children and Adolescents Are Not Learning Worldwide (Fact Sheet No. 46). Montréal : Institut de statistique de l'UNESCO (ISU). http://uis. unesco.org/sites/default/files/documents/fs46-more-than-half-children-not-learning-en-2017.pdf

Institut de statistique de l'UNESCO (ISU) (2017g). Rapid Data Quality Assessment for Pacific Island Countries. https://docs.google.com/forms/d/e/1FAlpQLSes2Bglll2fe-0cgR1p9GFhErh-zZd4GbVYNZ5WAAwhl5IWRw/ viewform?c=0\&w=1 (Consulté le 7 septembre 2017).

Institut de statistique de I'UNESCO (ISU) (2017h). SDG Data Reporting Proposal of a Protocol for Reporting Indicator 4.1.1. Montréal : Institut de statistique de l'UNESCO (ISU). http://uis.openplus.ca/gaml/files/ meeting4/Reporting_indicator_4.1.1.pdf

Institut de statistique de I'UNESCO (ISU) (2017i). The Data Revolution in Education (Information Paper No. 39). Montréal : Institut de statistique de I'UNESCO (ISU).

Institut de statistique de l'UNESCO (ISU) (2017j). UIS Indicator Development in the Field of Education (Information Paper No. 45). Montréal : Institut de statistique de I'UNESCO (ISU). http://uis.unesco.org/sites/ default/files/documents/ip45-indicator-development-education-methodology-2017-en.pdf

Institut de statistique de l'UNESCO (ISU) (à paraître). Code of Practice for Ministries of Education in Charge of Statistics Produced and Disseminated through Administrative Routine Data Systems. Montréal : Institut de statistique de I'UNESCO (ISU).

Institut de statistique de I'UNESCO (ISU) et ACER (2017). Principles of Good Practice in Learning Assessment. Montréal : Institut de statistique de l'UNESCO (ISU) and Australian Council for Educational Research (ACER). http://uis.unesco.org/sites/default/files/documents/principles-good-practice-learning-assessments2017-en.pdf

Institut de statistique de I'UNESCO (ISU) and ACER (à paraître). SDG 4 Reporting: Data Alignment Process. Montréal: Institut de statistique de l'UNESCO (ISU).

Keijzer, N. et Klingebiel, S. (2017). Realising the Data Revolution for Sustainable Development: Towards Capacity Development 4.0 (Document de travail No. 9). Paris : Partenariat en statistique pour le développement au 21ème siècle (PARIS21).

Montoya, S. et Hastedt, D. (2017). "News from Hamburg: Big Steps Forward Towards Reliable Metrics to Harmonise Learning Assessment Data Globally", UIS Data Blog. http://www.norrag.org/news-hamburg-bigsteps-forward-towards-reliable-metrics-harmonise-learning-assessment-data-globally-silvia-montoya-dirkhastedt/ 
Montoya, S. et Naidoo, J. (2017). "Moving up a Gear: The CapED Initiative", UIS Data Blog. https://sdg.uis. unesco.org/2017/08/02/moving-up-a-gear-the-caped-initiative/

OCDE (2015). OECD Assessment of the Statistical System and Key Statistics of Colombia. Paris: Organisation de coopération et de développement économiques (OCDE). http://www.oecd.org/std/OECD-Assessmentof-the-Statistical-System-and-Key-Statistics-of-Colombia.pdf

ONU (2000). Déclaration du Millénaire des Nations Unies (No. A/RES/55/2). New York : Organisation des Nations Unies. http://www.un.org/millennium/declaration/ares552e.pdf (Consultée le 7 septembre 2017).

ONU (2014). Principes fondamentaux de la statistique officielle (Résolution A/68/L.36, Assemblée générale, 29 janvier 2014). New York, NY : Organisation des Nations Unies. https://unstats.un.org/unsd/dnss/gp/FPNew-F.pdf

ONU (2015). Transformer notre monde : le Programme de développement durable à l'horizon 2030. New York : Organisation des Nations Unies. https://sustainabledevelopment.un.org/post2015/ transformingourworld/publication

ONU (2017). Forum politique de haut niveau des Nations unies sur le développement durable. https:// sustainabledevelopment.un.org/hlpf (Consulté le 7 septembre 2017).

PARIS21 (2017). Sectoral strategies for the Development of Statistics (SSDS) - NSDS Guidelines. Paris, France : Partenariat en statistique pour le développement au 21ème siècle (PARIS21). http://nsdsguidelines. paris21.org/node/292

TCG (2017). Agreement on indicators to monitor progress towards SDG 4 in 2017: Table 1 (Résultat de la seconde réunion du Groupe de coopération technique, Madrid, 28 octobre 2016). Madrid : Groupe de coopération technique sur les indicateurs pour l' ODD 4-Éducation 2030 (TCG). http://uis.unesco.org/sites/ default/files/documents/tcg-thematic-education-indicators-final.xlsx

UNESCO (2000). Le Cadre d'action de Dakar. L'éducation pour tous : tenir nos engagements collectifs. Comprenant six Cadres d'action régionaux. Texte adopté par le Forum sur l'éducation pour tous. Dakar, 2628 avril. Paris: UNESCO.

UNESCO (2015). Education 2030 Déclaration d'Incheon et Cadre d'action pour la mise en œuvre de l'Objectif de développement durable 4 Assurer à tous une éducation équitable, inclusive et de qualité et des possibilités d'apprentissage tout au long de la vie (No. ED-2016/WS/2). Paris : UNESCO.

UNESCO (2016). The CapED Annual Report 2016. Paris : UNESCO.

UNESCO (2017). Stratégie à moyen-terme révisée 2017-2021 de l'Institut de statistique de l'UNESCO (Deux cent-unième session du Conseil exécutif de l'UNESCO, point 13 de l'ordre du jour provisoire No. 201 EX/13). Paris : UNESCO.

UNGA (2014). Principes fondamentaux de la statistique officielle (Principes fondamentaux de la statistique officielle nationale No. A/RES/68/261). New York : Assemblée générale des Nations Unies (UNGA). https:// unstats.un.org/unsd/dnss/gp/FP-New-F.pdf (Consulté le 13 décembre 2017).

UNSG (2014). "La dignité pour tous d'ici à 2030 : éliminer la pauvreté, transformer nos vies et protéger la planète", rapport de synthèse du Secrétaire général sur le programme de développement durable pour l'après-2015 (No. A/69/700). New York : Secrétaire général des Nations Unies (UNSG). http://www.un.org/ ga/search/view_doc.asp?symbol=A/69/700\&Lang=F (Consulté le 7 septembre 2017).

UNSG (2016). Rapport du Groupe d'experts des Nations Unies et de l'extérieur chargé des indicateurs relatifs aux objectifs de développement durable (Commission de statistique, 8-11 mars 2016 Point 3 a) de l'ordre du jour provisoire. Questions soumises à la Commission pour examen et décision : données et indicateurs relatifs au Programme de développement durable à l'horizon 2030 No. E/CN.3/2016/2/Rev.1*). New York : Secrétaire général des Nations Unies (UNSG) et Conseil économique et social (ECOSOC). http://undocs. org/fr/E/CN.3/2016/2/Rev.1 


\section{Annexe 1. Code de pratiques pour les statistiques de l'éducation : Principes et indicateurs}

\section{Environnement institutionnel}

Les facteurs institutionnels et organisationnels ont une influence significative sur l'efficacité et la crédibilité des ministères de l'éducation qui élaborent, produisent et diffusent les statistiques de l'éducation.

\section{Principe 1 : Cadre politique et juridique.} L'environnement juridique et institutionnel qui régit les statistiques de l'éducation a une influence significative sur l'efficacité et la crédibilité du ministère de l'éducation qui produit et diffuse les statistiques de l'éducation.

1.1: La responsabilité de la collecte, du traitement et de la diffusion des statistiques est clairement spécifiée.

1.2: Le partage des données et la coordination entre les agences qui produisent les données sur l'éducation sont appropriés.

1.3: Les données des répondants doivent rester confidentielles et n'être utilisées qu'à des fins statistiques.

1.4: La communication des données statistiques est assurée par le biais de mesures et/ou d'un mandat juridique qui encourage(nt) à répondre.

Principe 2. Adéquation des ressources. Le ministère de l'éducation s'assure que les ressources sont proportionnées aux programmes statistiques, personnels, installations, équipements, technologies, formations et financements de leurs systèmes de gestion des informations relatives à l'éducation.

2.1: Les ressources en personnels, financières et informatiques sont proportionnées aux programmes statistiques du ministère de l'éducation.

2.2: Des mesures visant à s'assurer que l'utilisation des ressources est efficient sont mises en œuvre.

Principe 3. Sensibilisation à la qualité. La qualité est la clé de voûte des activités statistiques. Les ministères de l'éducation identifient régulièrement et systématiquement les points forts et les points faibles afin d'améliorer en permanence la qualité du traitement et des produits.
3.1: Des processus sont en place pour privilégier la qualité.

3.2: Les responsables prennent dûment en considération la qualité de la collecte, du traitement et de la diffusion des statistiques.

3.3: Les responsables intègrent les considérations liées à la qualité dans la planification du programme statistique.

Principe 4. Professionnalisme. Les politiques et les pratiques statistiques sont guidées par des principes professionnels.

4.1: Les statistiques sont compilées d'une façon impartiale.

4.2: Les choix des sources et des techniques statistiques ne sont guidés que par des considérations statistiques.

4.3: L'entité statistique appropriée est habilitée à commenter les interprétations erronées et les mauvais usages de statistiques.

Principe 5. Transparence. Les ministères de l'éducation élaborent, produisent et diffusent les statistiques de l'éducation d'une façon objective et transparente, de sorte que tous les utilisateurs soient traités équitablement.

5.1: Les modalités de la collecte, du traitement et de la diffusion des statistiques sont à la disposition du public.

5.2: L'accès gouvernemental interne aux statistiques avant leur publication est publiquement identifié.

5.3: Les produits des organismes/unités statistiques sont clairement identifiés en tant que tels.

5.4: Les utilisateurs sont informés à l'avance de tout changement majeur apporté à la méthodologie, aux données sources et aux techniques statistiques.

Principe 6. Normes éthiques. Les politiques et les pratiques sont guidées par des normes éthiques.

6.1: Des principes directeurs relatifs au comportement du personnel sont en place et bien connus du personnel. 


\section{Processus statistiques}

Les normes, principes directeurs et bonnes pratiques internationaux sont pleinement respectés dans les processus utilisés par les ministères pour organiser, collecter, traiter et diffuser les statistiques officielles. La crédibilité des statistiques est renforcée par une réputation de bonne gestion et d'efficience.

Principe 7. Solidité de la méthodologie. La base méthodologique des statistiques de l'éducation suit les normes, principes directeurs ou bonnes pratiques acceptés au plan international.

7.1: Les concepts et les définitions utilisés sont conformes aux cadres statistiques standards.

7.2: La portée des statistiques de l'éducation est conforme aux normes, principes directeurs ou bonnes pratiques acceptés au plan international.

7.3: Les systèmes de classification sont conformes aux normes, principes directeurs ou bonnes pratiques acceptés au plan national et international.

7.4: Les données sont enregistrées conformément aux normes, principes directeurs ou bonnes pratiques acceptés au plan international.

Principe 8. Exactitude et fiabilité. Les sources des données et les techniques statistiques sont solides et les résultats des statistiques de l'éducation reflètent la réalité de façon appropriée.

8.1: Les sources de données disponibles constituent une base appropriée pour compiler les statistiques.

8.2: Les sources de données sont régulièrement évaluées et validées.

8.3: Les techniques statistiques utilisées sont conformes à de solides procédures statistiques et sont documentées.

8.4: Les révisions, qui permettent de mesurer la fiabilité, sont surveillées et exploitées pour les informations qu'elles peuvent fournir.

8.5: Les données sources et les résultats statistiques sont archivés.

\section{Résultats des statistiques de l'éducation}

Les statistiques disponibles répondent aux besoins des utilisateurs. Les statistiques de l'éducation sont conformes aux normes internationales relatives à la qualité et répondent aux besoins des institutions internationales, gouvernements, instituts de recherche, préoccupations des entreprises et au grand public.

Principe 9. Pertinence. Les statistiques de l'éducation répondent aux besoins des utilisateurs.

9.1: Les utilisateurs de données sont régulièrement consultés.

9.2: Les besoins prioritaires sont satisfaits et pris en compte dans le programme de travail.

9.3: La satisfaction des utilisateurs est régulièrement surveillée et fait l'objet d'un suivi systématique.

Principe 10. Périodicité et respect des délais. Les statistiques de l'éducation sont publiées conformément à une périodicité et à un calendrier acceptés au plan international.

10.1: La périodicité suit des normes de diffusion.

10.2: La publication en temps voulu suit des normes de diffusion.

10.3: Un calendrier des publications est mis à disposition et les écarts par rapport au calendrier de diffusion sont rendus publics.

Principe 11. Cohérence. Les statistiques de l'éducation publiées sont cohérentes dans un ensemble de données et, au fil du temps, dans d'autres ensembles de données majeurs.

11.1: Les statistiques finales sont cohérentes dans un ensemble de données.

11.2: Les statistiques finales sont cohérentes ou réconciliables pendant un délai raisonnable.

11.3: Les statistiques finales sont cohérentes ou réconciliables avec celles obtenues par l'intermédiaires d'autres enquêtes et sources de données.

Principe 12. Accessibilité et clarté. Les statistiques et les métadonnées de l'éducation sont aisément accessibles, présentées d'une façon claire et compréhensible et le soutien aux utilisateurs est approprié.

12.1: Les statistiques sont présentées d'une façon claire et compréhensible, les formes de diffusion sont appropriées, et les statistiques sont mises à disposition de façon impartiale.

12.2: Des métadonnées à jour et pertinentes sont mises à disposition.

12.3: Des services de soutien aux utilisateurs rapides et compétents sont disponibles. 


\section{Annexe 2. Liens entre le Code de pratiques de l'ISU pour les statistiques de l'éducation et le Cadre national d'assurance de la qualité (NQAF)}

Environnement institutionnel : Les facteurs institutionnels et organisationnels ont une influence significative sur l'efficacité et la crédibilité des ministères de l'éducation qui élaborent, produisent et diffusent les statistiques de l'éducation. Les aspects pertinents sont le cadre politique et juridique, l'adéquation des ressources, la sensibilisation à la qualité, le professionnalisme, la transparence et les normes éthiques.

\section{Prérequis de la qualité \\ L'environnement institutionnel garantit l'efficacité des principes fondamentaux des statistiques officielles.}

Principe 1 : Cadre politique et juridique. L'environnement juridique et institutionnel régissant les statistiques de l'éducation a une influence significative sur l'efficacité et la crédibilité du ministère de l'éducation qui produit et diffuse les statistiques de l'éducation.
NQAF01. Coordonner le système statistique national NQAF07. Assurer la confidentialité et la sécurité des statistiques

NQAF13. Gérer la charge des répondants

Principe 2 : Adéquation des ressources. Le ministère de l'éducation s'assure que les ressources sont proportionnées aux programmes statistiques, personnels, installations, équipements, technologies, formations et financements de leurs systèmes de gestion des informations relatives à l'éducation.

Principe 3 : Sensibilisation à la qualité. La qualité est la clé de voûte des activités statistiques. Les ministères de l'éducation identifient régulièrement et systématiquement les points forts et les points faibles afin d'améliorer en permanence la qualité du traitement et des produits.

\section{Assurances de l'intégrité}

Hay una adhesión firme al principio de objetividad en la recolección, el procesamiento y la difusión de estadísticas. Le principe d'objectivité de la collecte, du traitement et de la diffusion des statistiques est strictement respecté.

Principe 4 : Professionnalisme. Les politiques et les pratiques statistiques sont guidées par des principes professionnels.

QAF05. Assurer l'impartialité et l'objectivité NQAF04. Assurer l'indépendance professionnelle

Principe 5 : Transparence. Les ministères de l'éducation élaborent, produisent et diffusent les statistiques de l'éducation d'une façon objective et transparente, de sorte que tous les utilisateurs soient

NQAF06. Assurer la transparence traités équitablement.

Principe 6 : Normes éthiques. Les politiques et les pratiques sont guidées par des normes éthiques.

NQAF05. Assurer l'impartialité et l'objectivité 
Processus statistiques : Les normes, principes directeurs et bonnes pratiques internationaux sont pleinement respectés dans les processus utilisés par les ministères pour organiser, collecter, traiter et diffuser les statistiques officielles. La crédibilité des statistiques est renforcée par une réputation de bonne gestion et d'efficience. Les aspects pertinents sont la solidité méthodologique, l'exactitude et la fiabilité.

\section{Solidité méthodologique}

La base méthodologique des statistiques de l'éducation suit les normes, principes directeurs ou bonnes pratiques acceptés au plan international.

Principe 7 : Solidité de la méthodologie. La base méthodologique des statistiques de l'éducation suit les normes, principes directeurs ou bonnes pratiques acceptés au plan international.
NQAF10. Assurer la solidité méthodologique

\section{Exactitude et fiabilité \\ Les sources des données et les techniques statistiques sont solides et les résultats des statistiques de l'éducation reflètent la réalité de façon appropriée.}

Principe 8 : Exactitude et fiabilité. Les sources des données et les techniques statistiques sont solides et les résultats des statistiques de l'éducation reflètent la réalité de façon appropriée.
NQAF03. Gérer les normes statistiques NQAF12. Assurer la solidité de la mise en œuvre NQAF15. Assurer l'exactitude et la fiabilité NQAF17. Assurer l'accessibilité et la clarté

Résultats des statistiques de l'éducation : Les statistiques disponibles répondent aux besoins des utilisateurs. Les statistiques de l'éducation sont conformes aux normes internationales relatives à la qualité et répondent aux besoins des institutions internationales, gouvernements, instituts de recherche, préoccupations des entreprises et au grand public.

\section{Fonctionnement}

Les statistiques sont pertinentes, leur périodicité et leur publication en temps opportun sont appropriées, elles sont cohérentes et elles suivent une politique prévisible en matière de révision.

Principe 9 : Pertinence. Les statistiques de l'éducation répondent aux besoins des utilisateurs.

NQAF14. Assurer la pertinence

Principe 10 : Périodicité et respect des délais. Les statistiques de l'éducation sont publiées conformément à une périodicité et à un calendrier acceptés au plan international.

NQAF16. Assurer le caractère opportun et la ponctualité

Principe 11 : Cohérence. Les statistiques de l'éducation publiées sont cohérentes dans un ensemble de données et, au fil du temps, dans d'autres ensembles de données majeurs.

\section{Accessibilité}

Les statistiques et les métadonnées de l'éducation sont aisément accessibles et le soutien aux utilisateurs est approprié.

Principe 12 : Accessibilité et clarté. Les statistiques et les métadonnées de l'éducation sont aisément accessibles, présentées d'une façon claire et compréhensible et le soutien aux utilisateurs est approprié.
NQAF17. Assurer l'accessibilité et la clarté

NQAF02. Gérer les relations avec les utilisateurs de données et avec les fournisseurs de données

Note : Les principes utilisés dans le NQAF de l'ONU sont ombrés. 


\section{Annexe 3. Stratégies de mise en œuvre de développement des capacités nationales}

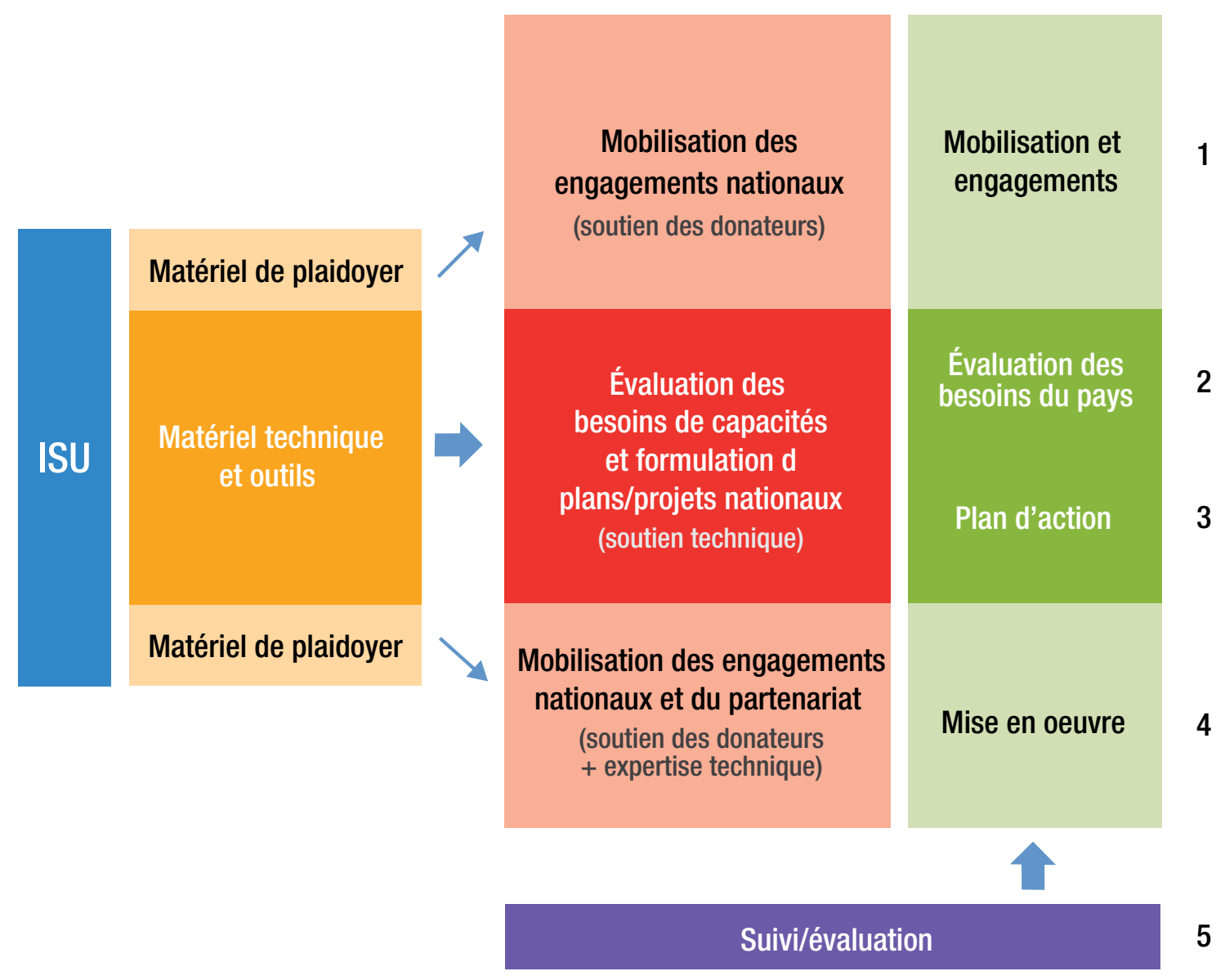




\section{Annexe 4. Cibles de l'ODD 4 par source des données}

\begin{tabular}{|c|c|c|c|c|}
\hline \multirow[b]{2}{*}{ Cibles } & \multicolumn{4}{|c|}{ Source des données } \\
\hline & $\begin{array}{l}\text { Adminis- } \\
\text { trative }\end{array}$ & Financière & $\begin{array}{l}\text { Enquête } \\
\text { auprès des } \\
\text { ménages }\end{array}$ & $\begin{array}{l}\text { Évaluation des } \\
\text { résultats } \\
\text { d'apprentissage }\end{array}$ \\
\hline $\begin{array}{l}\text { Cible } 4.1 \text { D'ici à } 2030 \text {, faire en sorte que toutes les filles et tous les garçons suivent, } \\
\text { sur un pied d'égalité, un cycle complet d'enseignement primaire et secondaire gratuit } \\
\text { et de qualité, les dotant d'acquis véritablement utiles. }\end{array}$ & 4 & & 3 & 1 \\
\hline $\begin{array}{l}\text { Cible } 4.2 \text { D'ici à } 2030 \text {, faire en sorte que toutes les filles et tous les garçons aient } \\
\text { accès à des activités de développement et de soins de la petite enfance et à une } \\
\text { éducation préscolaire de qualité qui les préparent à suivre un enseignement primaire }\end{array}$ & 3 & & 4 & 1 \\
\hline $\begin{array}{l}\text { Cible } 4.3 \text { D'ici à 2030, faire en sorte que les femmes et les hommes aient tous accès } \\
\text { dans des conditions d'égalité à un enseignement technique, professionnel ou tertiaire, } \\
\text { y compris universitaire, de qualité et d'un coût abordable }\end{array}$ & 3 & & 3 & \\
\hline $\begin{array}{l}\text { Cible 4.4 D'ici à 2030, augmenter considérablement le nombre de jeunes et d'adultes } \\
\text { disposant des compétences, notamment techniques et professionnelles, nécessaires } \\
\text { à l'emploi, à l'obtention d'un travail décent et à l'entrepreneuriat }\end{array}$ & & & 3 & 1 \\
\hline $\begin{array}{l}\text { Cible 4.5 D'ici à 2030, éliminer les inégalités entre les sexes dans le domaine de } \\
\text { l'éducation et assurer l'égalité d'accès des personnes vulnérables, y compris les } \\
\text { personnes handicapées, les autochtones et les enfants en situation vulnérable, à tous } \\
\text { les niveaux d'enseignement et de formation professionnelle }\end{array}$ & 3 & 2 & 1 & \\
\hline $\begin{array}{l}\text { Cible } 4.6 \text { D'ici à } 2030 \text {, veiller à ce que tous les jeunes et une proportion considérable } \\
\text { d'adultes, hommes et femmes, sachent lire, écrire et compter }\end{array}$ & 1 & & 3 & 1 \\
\hline $\begin{array}{l}\text { Cible 4.7 D'ici à 2030, faire en sorte que tous les élèves acquièrent les connaissances } \\
\text { et compétences nécessaires pour promouvoir le développement durable, notamment } \\
\text { par l'éducation en faveur du développement et de modes de vie durables, des droits } \\
\text { de l'homme, de l'égalité des sexes, de la promotion d'une culture de paix et de non- } \\
\text { violence, de la citoyenneté mondiale et de l'appréciation de la diversité culturelle et } \\
\text { de la contribution de la culture au développement durable }\end{array}$ & 3 & & & 2 \\
\hline $\begin{array}{l}\text { Cible 4.a Faire construire des établissements scolaires qui soient adaptés aux } \\
\text { enfants, aux personnes handicapées et aux deux sexes ou adapter les établissements } \\
\text { existants à cette fin et fournir un cadre d'apprentissage effectif qui soit sûr, exempt de } \\
\text { violence et accessible à tous }\end{array}$ & 1 & & 1 & \\
\hline $\begin{array}{l}\text { Cible 4.b D'ici à 2030, augmenter considérablement à l'échelle mondiale le nombre } \\
\text { de bourses d'études offertes aux pays en développement, en particulier aux pays les } \\
\text { moins avancés, aux petits États insulaires en développement et aux pays d'Afrique, } \\
\text { pour financer le suivi d'études supérieures, y compris la formation professionnelle, les } \\
\text { cursus informatiques, techniques et scientifiques et les études d'ingénieur, dans des } \\
\text { pays développés et d'autres pays en développement }\end{array}$ & 2 & & & \\
\hline $\begin{array}{l}\text { Cible 4.c D'ici à 2030, accroitre considérablement le nombre d'enseignants } \\
\text { qualifiés, notamment au moyen de la coopération internationale pour la formation } \\
\text { d'enseignants dans les pays en développement, surtout dans les pays les moins } \\
\text { avancés et les petits États insulaires en développement }\end{array}$ & 7 & & 1 & \\
\hline TOTAL ODD 4 & 27 & 2 & 19 & 6 \\
\hline
\end{tabular}




\section{Annexe 5. Douze questions principales pour évaluer la qualité des données des systèmes de finances publiques}

\begin{tabular}{|c|c|c|}
\hline & & Principe 3 : Pertinence \\
\hline \multirow{2}{*}{$\begin{array}{l}\text { Environnement } \\
\text { institutionnel }\end{array}$} & 1 & $\begin{array}{l}\text { Les utilisateurs de données du secteur de l'éducation sont systématiquement consultés ou informés } \\
\text { sur les aspects des données relatives aux finances publiques qui sont pertinentes pour leur secteur } \\
\text { (par exemple, classification, périodicité, caractère opportun). }\end{array}$ \\
\hline & 2 & $\begin{array}{l}\text { La production de données relatives au financement de l'éducation est coordonnée entre les différents } \\
\text { ministères (aux niveaux central, régional et local) et organismes impliqués dans le financement de } \\
\text { l'éducation. }\end{array}$ \\
\hline \multicolumn{3}{|r|}{ Principe 4 : Solidité de la méthodologie } \\
\hline \multirow{7}{*}{$\begin{array}{l}\text { Processus } \\
\text { statistiques }\end{array}$} & 3 & $\begin{array}{l}\text { Les processus de collecte et de publication des données relatives aux finances publiques couvrent } \\
\text { tous les niveaux de gouvernement (central, régional et local) d'un système de données centralisé. }\end{array}$ \\
\hline & 4 & $\begin{array}{l}\text { Les données relatives aux finances publiques comprennent une classification fonctionnelle (par } \\
\text { secteur tel que l'éducation, et pas uniquement par ministère ou par organisme), y compris de } \\
\text { l'éducation par niveaux. }\end{array}$ \\
\hline & 5 & $\begin{array}{l}\text { Les données relatives aux finances publiques comprennent une ventilation par type de dépense, y } \\
\text { compris les indemnisations des personnels enseignants et non enseignants, les dépenses consacrées } \\
\text { aux livres scolaires et matériels pédagogiques, les prêts aux étudiants et les bourses, et le soutien } \\
\text { apporté par le gouvernement aux établissements d'enseignement privés. }\end{array}$ \\
\hline & 6 & $\begin{array}{l}\text { Les données relatives aux finances publiques comprennent le détail des financements octroyés par } \\
\text { des donateurs étrangers qui sont budgétisés. }\end{array}$ \\
\hline & 7 & $\begin{array}{l}\text { Il existe une source de données utilisable et accessible pour les projets mis en œuvre grâce à des } \\
\text { donateurs étrangers (y compris ceux qui ne sont pas enregistrés dans le budget du gouvernement). }\end{array}$ \\
\hline & 8 & Il y a une source de données unifiée et reconnue relative aux dépenses publiques. \\
\hline & 9 & $\begin{array}{l}\text { Les données relatives aux finances publiques sont classées conformément au manuel des Statistiques } \\
\text { des finances publiques (GFS) ou à une classification régionale des dépenses publiques. }\end{array}$ \\
\hline \multicolumn{3}{|r|}{ Principe 6 : Périodicité et caractère opportun } \\
\hline \multirow{2}{*}{$\begin{array}{l}\text { Résultats } \\
\text { statistiques }\end{array}$} & 10 & $\begin{array}{l}\text { Les données relatives aux dépenses consacrées à l'éducation sont disponibles pour le budget et les } \\
\text { dépenses effectives de l'exercice fiscal précédent. }\end{array}$ \\
\hline & 11 & $\begin{array}{l}\text { Les données sur le financement de l'éducation comparables au plan international sont communiquées } \\
\text { en temps opportun. }\end{array}$ \\
\hline \multicolumn{3}{|r|}{ Principe 8 : Accessibilité et clarté } \\
\hline $\begin{array}{l}\text { Résultats } \\
\text { statistiques }\end{array}$ & 12 & $\begin{array}{l}\text { Les données relatives aux finances publiques du secteur de l'éducation sont diffusées et/ou mises à } \\
\text { la disposition des ministères de l'éducation d'une façon qui facilite leur accès (par exemple, en ligne, } \\
\text { accès à une base de données). }\end{array}$ \\
\hline
\end{tabular}

Source : Institut de statistique de l'UNESCO, 2017d. 



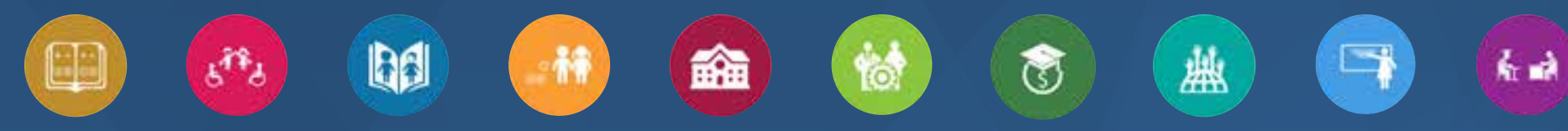

Les pays déploient d'énormes efforts pour répondre à la demande des données de plus en plus nombreuses et de qualité toujours supérieure associées aux objectifs de développement durable à l'horizon 2030. Dans le domaine de l'éducation, la communauté internationale est convenue d'utiliser un ensemble de 11 indicateurs mondiaux et de 32 indicateurs thématiques pour surveiller les progrès réalisés sur la voie de l'Objectif de développement durable (ODD) 4. Cependant, le défi consistant à les produire est immense. Selon les bases de données mondiales sur l'éducation les plus complètes au monde produites par l'Institut de statistique de I'UNESCO (ISU) - moins de la moitié des pays communiquent actuellement sur 19 indicateurs mondiaux et thématiques et seulement 1 indicateur mondial et 6 indicateurs thématiques sont couverts à plus de $75 \%$.

En tant que source officielle des données pour le Cadre d'action Éducation 2030ODD 4, l'ISU travaille quotidiennement avec les instituts de statistique nationaux et les ministères de l'éducation du monde entier pour collecter les données et appliquer les normes et les méthodologies nécessaires pour produire des indicateurs comparables au plan international. L'Institut comprend les défis auxquels sont confrontés les statisticiens nationaux et est donc remarquablement bien placé pour aider les pays à renforcer leurs capacités statistiques.

Le présent rapport présente une stratégie et un cadre complets pour améliorer la qualité des données nécessaires pour mettre en œuvre et surveiller les progrès sur la voie du Cadre d'action Éducation 2030-ODD 4. II montre un ensemble concret d'outils conçus pour aider les pays à renforcer chacune des étapes de leurs systèmes statistiques nationaux sur l'éducation - des outils de diagnostic pour évaluer la disponibilité et la qualité des données aux cadres et principes directeurs nécessaires pour initier une stratégie nationale visant à élaborer des statistiques de l'éducation qui répondent directement aux priorités politiques. Le rapport s'intéresse également aux questions plus larges du renforcement des capacités statistiques auxquels doivent faire face les pays, les donateurs et les partenaires. Surtout, l'ISU montre la voie pour construire des systèmes statistiques de meilleure qualité en fournissant aux parties prenantes nationales les outils et les stratégies dont ils ont besoin pour produire des données de grande qualité et atteindre leurs objectifs en matière de développement.

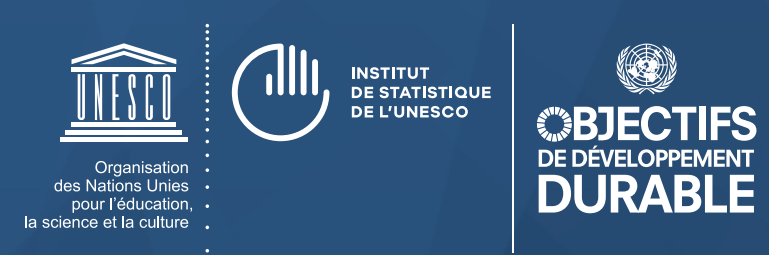

ISBN 978-92-9189-226-6

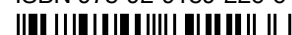

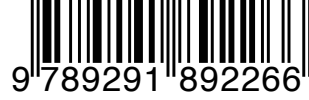

Institut de statistique de l'UNESCO

C.P. 6128 , Succursale Centre-Ville

Montréal, Québec H3C 3J7

Canada 